MATHEMATICS OF COMPUTATION

Volume 67, Number 221, January 1998, Pages 137-182

S 0025-5718(98)00911-9

\title{
CONVERGENCE OF A NON-STIFF BOUNDARY INTEGRAL METHOD FOR INTERFACIAL FLOWS WITH SURFACE TENSION
}

\author{
HÉCTOR D. CENICEROS AND THOMAS Y. HOU
}

\begin{abstract}
Boundary integral methods to simulate interfacial flows are very sensitive to numerical instabilities. In addition, surface tension introduces nonlinear terms with high order spatial derivatives into the interface dynamics. This makes the spatial discretization even more difficult and, at the same time, imposes a severe time step constraint for stable explicit time integration methods.

A proof of the convergence of a reformulated boundary integral method for two-density fluid interfaces with surface tension is presented. The method is based on a scheme introduced by Hou, Lowengrub and Shelley [ J. Comp. Phys. 114 (1994), pp. 312-338] to remove the high order stability constraint or stiffness. Some numerical filtering is applied carefully at certain places in the discretization to guarantee stability. The key of the proof is to identify the most singular terms of the method and to show, through energy estimates, that these terms balance one another.

The analysis is at a time continuous-space discrete level but a fully discrete case for a simple Hele-Shaw interface is also studied. The time discrete analysis shows that the high order stiffness is removed and also provides an estimate of how the CFL constraint depends on the curvature and regularity of the solution.

The robustness of the method is illustrated with several numerical examples. A numerical simulation of an unstably stratified two-density interfacial flow shows the roll-up of the interface; the computations proceed up to a time where the interface is about to pinch off and trapped bubbles of fluid are formed. The method remains stable even in the full nonlinear regime of motion. Another application of the method shows the process of drop formation in a falling single fluid.
\end{abstract}

\section{INTRODUCTION}

Boundary integral methods are a popular choice for simulations of interfacial flows and have been used extensively to compute nonlinear surface wave [6], [19], [30], [35], [37], vortex sheet (no density difference) motion [3], [23], [26], [27], [36], [38], Rayleigh-Taylor instability [5], [35], [44], interfaces in Hele-Shaw cells [17], [24], and crystal growth and solidification [29], [40]. The advantage of boundary integral

Received by the editor December 7, 1995 and, in revised form, June 5, 1996.

1991 Mathematics Subject Classification. Primary 65M12, 76B15.

Key words and phrases. boundary integral method, surface tension, stiffness.

The first author was partially supported by the Office of Naval Research under Grant N0001494-1-0310.

The second author was partially supported by the Office of Naval Research under Grant N00014-94-1-0310 and the National Science Foundation under grant DMS-9407030. 
methods is that they reduce the dimension of the problem by involving quantities along the interface only [34]. However, boundary integral methods designed for computing interface motion are very sensitive to numerical instabilities because the underlying problem is fairly singular. If left uncontrolled, these instabilities usually destroy the accuracy of the computations.

Numerical instabilities have been observed by Longuet-Higgins and Cokelet [30], Baker, Meiron and Orszag [6], and Dold [19] in surface wave simulations without surface tension. In these simulations, the onset of the instability was delayed with the application of polynomial averaging smoothing techniques. It is important to note, however, that these ad hoc smoothing techniques do not completely eliminate the numerical instability.

Computations of interfacial flows with surface tension are even more sensitive to numerical instabilities because surface tension introduces nonlinear terms with high order spatial derivatives into the interface evolution equations. Straightforward discretizations of the surface tension term may lead to numerical instabilities. Pullin [35] was one of the first to experience this difficulty. Using a boundary integral method for computing two fluid interfacial flows, Pullin noted small-scale corrugations in regions of high curvature in the interface profile that appeared only with nonzero surface tension, in addition to a sawtooth instability of the type described by Longuet-Higgins and Cokelet [30]. While he could partially delay the sawtooth instability with polynomial averaging, eventually the appearance of the first type of numerical instability, associated with surface tension, caused the computations to end.

Using also a boundary integral method, Rangel and Sirignano [36] limited the growth of modes with high wavenumbers by doing redistribution and point insertion of vortices. This procedure gives a smoothing effect very similar to that of the vortex-blob method [26] or the vortex-in-cell method used by Tryggvason [44] and it becomes very difficult to distinguish the real effects of surface tension with this regularization.

Linear analysis about equilibrium has helped to identify common sources of instability in boundary integral methods. Without surface tension, Roberts [37] showed how to remove a sawtooth instability.

In recent works $[4,7]$, numerical stability analyses were presented in two different contexts of interfacial flows with surface tension. Baker and Nachbin [4], performed normal mode analysis for several boundary integral schemes to study the linear evolution of periodic perturbations of a flat vortex sheet. With this linear analysis, Baker and Nachbin were able to identify common causes for numerical instability. However, their study did not consider the behavior of perturbations away from equilibrium and left open the questions of nonlinear stability and convergence.

Beale, Hou and Lowengrub [7], hereafter referred to as BHL, presented a convergence proof of a boundary integral for water waves with or without surface tension. Following a framework developed in [9] for linearized motion perturbed about an arbitrary smooth solution at the continuous level, BHL found that very delicate balances among terms with singular integrals and derivatives must be preserved at the discrete level in order to maintain numerical stability. They also realized that suitable numerical filtering is necessary at certain places to prevent the discretization from introducing new instabilities in the high modes. This filtering depends on the choices for approximating spatial derivatives and quadrature rules for singular 
integrals. Spectral methods are no exception. Indeed, the periodicity of the numerical solution introduces aliasing errors which ultimately become another source of instability and consequently, some Fourier filtering is also required in this case.

Another major difficulty in numerical simulations of interfacial flows with surface tension, is related to time discretizations. Surface tension introduces terms with high order derivatives both nonlinear and nonlocal into the interface dynamics. This leads to severe stability constraints for explicit time integration methods and makes the application of implicit methods difficult $[13,24]$. For two fluid interfaces in Euler flow, the stability constraint is of the form $\Delta t \leq C \cdot(\Delta s)^{3 / 2}$, and for HeleShaw flows is $\Delta t \leq C \cdot(\Delta s)^{3}$, where $\Delta t$ is the size of the time step and $\Delta s$ is the minimum grid spacing in arclength. In practice, these time-dependent constraints become more severe by point clustering along the interface. This imposes a serious limitation for well resolved computations. The presence of such restrictions on the time step is known as stiffness.

In [24], Hou, Lowengrub and Shelley, hereafter referred to as HLS, introduced methods that remove the high order stiffness in computing the motion of fluid interfaces with surface tension in two-dimensional, irrotational and incompressible fluids. Their schemes are based on a spectral discretization of a boundary integral reformulation using two natural variables: the tangent angle $\theta$ to the curve and the arclength stretching $\sigma$, which is the derivative of the arclength with respect to a Lagrangian marker $\alpha$. In these variables, the surface tension term has a very simple form because the local curvature becomes $\theta_{\alpha} / \sigma$. HLS identified the leading order stiff terms and treated them implicitly in time discretizations. These semi-implicit methods can be solved easily once a suitable parametrization of the interface is chosen. The methods are efficient and give an enormous saving in computational time [23].

In this paper, we present a method designed for general two-density fluid interfaces with surface tension in Euler flows, and study its nonlinear stability and convergence. The method is based on the spectral spatial discretization used by HLS, keeping time continuous. Thus, used in combination with the semi-implicit time discretizations proposed by HLS, our method also removes the high order stiffness introduced by surface tension.

Our analysis is at a time continuous-space discrete level but we also study a fully discrete case for a simple Hele-Shaw interface and show that the time step $\Delta t$ depends only linearly on the mesh size $h$, i.e., $\Delta t \leq C h$. Our analysis also indicates how the stability constant $C$ depends on the regularity of the solution, especially the curvature.

We apply certain Fourier filtering in the method at places suggested by our stability analysis to preserve the balance among most singular terms. With this filtering as part of the method, we are able to prove rigorously its convergence to the exact solution with spectral accuracy provided the solution is sufficiently regular. This is the content of Theorem 1.

Our proof follows a framework developed in $[8,10]$ to study the linearized motion about smooth solutions of two fluid interfaces with surface tension in the continuous case, in the same spirit of BHL's convergence proof of a method for water waves [7]. The key is to identify the leading order singular terms of the method and to make sure that a delicate balance among these terms is satisfied. However, the situation is more complicated than that in the water waves scheme studied by BHL. 
In our case, the formulation is different and more general because it applies to twodensity fluid interfaces of which water waves is a special case, and it uses $\sigma$ and $\theta$ as evolution variables to determine the interface position rather than the Cartesian coordinates $x$ and $y$.

By adding a tangential velocity, we select a parametrization such that, $\sigma=$ $R(\alpha)\langle\sigma\rangle$, where the brackets denote average in space and $R$ is a smooth function of mean one. $R$ can be chosen at our advantage to obtain some control on the positioning of the interface Lagrangian particles. Thus, we may cluster particles in neighborhoods of singular points (taking $R$ small there) and separate the particles in over-resolved regions. While this parametrization can give a substantial improvement in the resolution, it introduces new technical difficulties into the analysis. In particular, we find that a natural variable to use in the computation of the velocity is the true vortex sheet strength $\tilde{\gamma}=\gamma / \sigma$, where $\gamma$ is the unnormalized vortex sheet strength. By filtering $\tilde{\gamma}$ in the velocity, we are able to show, through delicate energy estimates, that the most singular terms of the method balance one another. We do not claim that the application of Fourier filtering to eliminate aliasing errors is unique but we do show that our choice gives a nonlinearly stable and convergent

discretization. The same ideas can be applied to construct nonlinearly stable and convergent methods for Hele-Shaw flows, and dendritic solidification problems.

The rest of the paper is organized as follows. Section 2 is devoted to describe the boundary integral reformulation introduced by HLS and their ideas to remove the high order stiffness. In Section 3, we present our numerical method and state the convergence result (Theorem 1). The proof of this theorem is given in Section 4. Finally, in Section 5, some numerical examples are included to demonstrate the robustness of the method. A numerical simulation of an unstably stratified twodensity interfacial flow shows the roll-up of the interface; the computations proceed up to a time where the interface is about to pinch off and trapped bubbles of fluid are formed. The method remains stable even in the full nonlinear regime of motion. Another application of the method shows the process of drop formation in a falling single fluid.

\section{A BOUNDARY INTEGRAL REFORMULATION FOR TWO FLUID INTERFACES}

In this section, we present the boundary integral reformulation introduced by HLS for two fluid interfaces and describe their ideas to remove the high order stiffness caused by surface tension. Specifically, we concentrate on the motion of an interface $\Gamma$ separating two infinite layers of inviscid, incompressible and irrotational fluids in two dimensions. Under these assumptions, potential flows exist in the interior of the layers and the flow can be described by variables defined on the interface only. The boundary conditions we impose are

$$
\begin{aligned}
& {[\boldsymbol{u}]_{\Gamma} \cdot \hat{\boldsymbol{n}}=0,} \\
& {[p]_{\Gamma}=\tau \kappa,}
\end{aligned}
$$

where $[\boldsymbol{u}]_{\Gamma} \cdot \hat{\boldsymbol{n}}$ and $[p]_{\Gamma}$ denote the jump in normal velocity and pressure across the interface, respectively. The constant $\tau$ is the surface tension coefficient and $\kappa$ the local curvature. The kinematic condition (i) is the usual requirement that particles on the surface, remain there. The dynamic condition (ii) is known as Laplace's formula [28]. With these hypotheses we can obtain a boundary integral formulation for the interface evolution equations. Here we give only the equations, 
the details of the derivation can be found in [6]. Denote the interface position by $\boldsymbol{X}(\alpha, t)=(x(\alpha, t), y(\alpha, t))$, where $\alpha$ is a Lagrangian parameter and let $\hat{\boldsymbol{n}}$ and $\hat{\boldsymbol{s}}$ be unit normal and tangential vectors to the interface. The mean velocity on the interface can be evaluated by the following Cauchy principal value integral

$$
\boldsymbol{W}=f_{-\infty}^{\infty} K\left(\boldsymbol{X}(\alpha)-\boldsymbol{X}\left(\alpha^{\prime}\right)\right) \gamma\left(\alpha^{\prime}\right) d \alpha^{\prime},
$$

where the singular kernel $K$ is given by

$$
K(\boldsymbol{X}(\alpha))=\frac{1}{2 \pi} \cdot \frac{(-y(\alpha), x(\alpha))}{x^{2}(\alpha)+y^{2}(\alpha)},
$$

and $\gamma$ is the unnormalized vortex sheet strength. Given $\gamma(\alpha, t)$ and $\boldsymbol{X}(\alpha, t)$, the interface normal velocity is determined by $U^{N}=\boldsymbol{W} \cdot \hat{\boldsymbol{n}}$ and this complies with the kinematic boundary condition (i). On the other hand, we have freedom to select the tangential velocity. We take this to be $U^{T}+U^{A}$, where $U^{T}=\boldsymbol{W} \cdot \hat{\boldsymbol{s}}$ and $U^{A}$ is yet arbitrary and its choice determines the frame of reference. To update the velocity we need an evolution equation for $\gamma$, this equation follows from the dynamic boundary condition (ii). The interface evolves according to the following equations

$$
\begin{aligned}
\boldsymbol{X}_{t} & =U^{N} \hat{\boldsymbol{n}}+\left(U^{T}+U^{A}\right) \hat{\boldsymbol{s}}, \\
\gamma_{t} & =S \kappa_{\alpha}+\left(\frac{U^{A} \gamma}{\sigma}\right)_{\alpha}-2 A\left[\sigma \boldsymbol{W}_{t} \cdot \hat{\boldsymbol{s}}+\frac{1}{8}\left(\frac{\gamma}{\sigma}\right)_{\alpha}^{2}+g y_{\alpha}-U^{A} \boldsymbol{W}_{\alpha} \cdot \hat{\boldsymbol{s}}\right],
\end{aligned}
$$

where $\sigma=\left(x_{\alpha}^{2}+y_{\alpha}^{2}\right)^{1 / 2}$ is the arclength stretching variable and $A=\left(\rho_{1}-\rho_{2}\right) /\left(\rho_{1}+\rho_{2}\right)$ is the Atwood number which measures the flow stratification. Here, $\rho_{1}\left(\rho_{2}\right)$ is the density of the fluid below (above) the interface. The constant $S=2 \tau /\left(\rho_{1}+\rho_{2}\right)$ is a rescaled surface tension coefficient and $g$ the gravity acceleration. The local curvature $\kappa$ is given by

$$
\kappa=\frac{y_{\alpha \alpha} x_{\alpha}-x_{\alpha \alpha} y_{\alpha}}{\left(x_{\alpha}^{2}+y_{\alpha}^{2}\right)^{3 / 2}} .
$$

The subindex $\alpha$ denotes partial differentiation with respect to that variable.

From this set of equations we observe that the surface tension enters into the interface dynamics terms with high (third) order derivatives nonlinearly and nonlocally, making implicit time discretizations very difficult.

A "frozen coefficient" linear analysis about an arbitrary smooth solution for the evolution equations (2)-(3) gives the following stability constraint for an explicit time integration method [13, 24],

$$
\Delta t<\frac{C}{S}(\bar{\sigma} h)^{3 / 2} \quad \text { with } \quad \bar{\sigma}=\min _{\alpha} \sigma .
$$

Although the exponent $3 / 2$ may not seem very restrictive, this stability constraint is still very severe because of its dependence on $\bar{\sigma}$. As the interface evolves, Lagrangian particles tend to cluster in some regions and cause $\bar{\sigma}$ to be very small. This timedependent behavior usually leads to prohibitively stiff systems.

The first step in the HLS nonstiff method is to reformulate the interface evolution equations in terms of the tangent angle $\theta$ and the arclength stretching $\sigma$. In these two new variables, the surface tension term has a very simple form: $S \kappa_{\alpha}=S\left(\theta_{\alpha} / \sigma\right)_{\alpha}$. This approach has been used previously by Kessler, Koplik and Levine [25] for interface evolution problems, by Strain [40] in the context of 
solidification, by Goldstein and Petrich [21] for integrable closed curve dynamics, and by Meiburg and Homsy [32] for Hele-Shaw flows.

If we assume that an interface moves according to $\boldsymbol{X}_{t}=U^{N} \hat{\boldsymbol{n}}+\left(U^{T}+U^{A}\right) \hat{\boldsymbol{s}}$, then the evolution equations for $\sigma$ and $\theta$ are given by (see, e.g., [15, 24])

$$
\begin{aligned}
\sigma_{t} & =\left(U^{T}+U^{A}\right)_{\alpha}-\theta_{\alpha} U^{N}, \\
\theta_{t} & =\frac{1}{\sigma}\left[U_{\alpha}^{N}+\theta_{\alpha}\left(U^{T}+U^{A}\right)\right] .
\end{aligned}
$$

Note that the evolution equations (4)-(5) can be used in many other different contexts depending on how the velocity is determined. Examples include motion by mean curvature, dendritic solidification and Hele-Shaw interfaces.

In the particular case of an interface in Euler flows the velocity $\boldsymbol{W} \equiv(U, V)$ defined in (1) has a succinct form when expressed in terms of the complex position variable $z=x+i y$,

$$
W=\frac{1}{2 \pi i} \int_{-\infty}^{\infty} \frac{\gamma\left(\alpha^{\prime}\right)}{z(\alpha)-z\left(\alpha^{\prime}\right)} d \alpha^{\prime}=U-i V .
$$

Moreover, if we assume that the interface is periodic in the $x$ direction, that is $z(\alpha)=\alpha+s(\alpha)$ with $s(\alpha)$ a $2 \pi$ periodic function, then it can be shown [2] that

$$
W=\frac{1}{4 \pi i} f_{-\pi}^{\pi} \gamma\left(\alpha^{\prime}\right) \cot \frac{1}{2}\left(z(\alpha)-z\left(\alpha^{\prime}\right)\right) d \alpha^{\prime} .
$$

The complex position $z$ may be obtained from $\sigma$ and $\theta$ by integrating $z_{\alpha}=\sigma e^{i \theta}$ or equivalently

$$
z(\alpha)=\alpha+\int_{0}^{\alpha}\left(\sigma\left(\alpha^{\prime}\right) e^{i \theta\left(\alpha^{\prime}\right)}-\left\langle\sigma e^{i \theta}\right\rangle\right) d \alpha^{\prime}+z_{0},
$$

where the brackets denote the mean or average in space over one period, that is

$$
\langle f\rangle \equiv \frac{1}{2 \pi} \int_{0}^{2 \pi} f\left(\alpha^{\prime}\right) d \alpha^{\prime},
$$

and we have also used that $\left\langle z_{\alpha}\right\rangle=1+\left\langle s_{\alpha}\right\rangle=1$, as $s(\alpha)$ is periodic. A discretization of (8) enforces the periodicity assumption on $z$ [24]. The integration constant $z_{0}$ can be determined by evolving one single point of the interface in time. When the two layers of fluid are infinite in the $y$-direction, $z_{0}$ provides just a translation to the curve and does not influence its dynamics. For simplicity we take $z_{0}=0$.

There is still freedom in selecting the additional tangential velocity $U^{A}$. Following HLS, we choose $U^{A}$ such that the arclength stretching variable satisfies the following condition,

$$
\sigma(\alpha, t)=R(\alpha)\langle\sigma\rangle,
$$

where $R(\alpha)$ is a given smooth function of mean one. This condition gives us some control on the positioning of the Lagrangian markers. In particular, if we know in advance where some complexity of the curve is located, $R$ can be selected to cluster particles about that region to achieve better resolution. It can be shown [15, 24] that if

$$
U^{A}=-U^{T}+\int_{0}^{\alpha}\left[\theta_{\alpha} U^{N}-R(\alpha)\left\langle\theta_{\alpha} U^{N}\right\rangle\right] d \alpha^{\prime},
$$

then this choice of $U^{A}$ enforces the constraint (9) for all time provided it is satisfied initially. 
We can now write the reformulated evolution equations of the interface in the following form,

$$
\begin{aligned}
\sigma_{t} & =-R(\alpha)\left\langle\theta_{\alpha} U^{N}\right\rangle, \\
\theta_{t} & =\frac{1}{\sigma}\left[U_{\alpha}^{N}+\theta_{\alpha}\left(U^{T}+U^{A}\right)\right], \\
\gamma_{t} & =S\left(\frac{\theta_{\alpha}}{\sigma}\right)_{\alpha}+\left(\frac{\gamma}{\sigma} U^{A}\right)_{\alpha} \\
& -2 A\left[\operatorname{Re}\left\{\sigma e^{i \theta} W_{t}\right\}+\frac{1}{8}\left(\frac{\gamma}{\sigma}\right)_{\alpha}^{2}+g \sigma \sin \theta-U^{A} \operatorname{Re}\left\{e^{i \theta} W_{\alpha}\right\}\right],
\end{aligned}
$$

where $U^{N}=-\operatorname{Im}\left\{e^{i \theta} W\right\}$ and $U^{T}=\operatorname{Re}\left\{e^{i \theta} W\right\}$ with $R e$ and $\operatorname{Im}$ denoting the real and imaginary parts respectively. Actually, we could cancel the parametrization factor $R(\alpha)$ in (11) and obtain an evolution equation for the average $\langle\sigma\rangle$. However, equation (11) allows for a more general setting.

The surface tension term $S\left(\theta_{\alpha} / \sigma\right)_{\alpha}$ affects the evolution of $\theta$ through the vortex sheet strength $\gamma$ in the velocity field. Thus, $U_{\alpha}^{N}$ is the term that contributes the most to the stiffness in (12). This nonlocal effect makes difficult the application of implicit time discretizations. However, an important fact is that stiffness arises from high order terms only at small spatial scales; the time step has to be small enough to resolve the propagation of capillary waves [13].

The next step in HLS approach is to extract the leading part of the velocity at small scales (high modes). To this end, we consider the expression (6) for $W$ and write the kernel as follows

$$
\frac{1}{z(\alpha)-z\left(\alpha^{\prime}\right)}=\frac{1}{z_{\alpha}(\alpha)\left(\alpha-\alpha^{\prime}\right)}+\left[\frac{1}{z(\alpha)-z\left(\alpha^{\prime}\right)}-\frac{1}{z_{\alpha}(\alpha)\left(\alpha-\alpha^{\prime}\right)}\right] .
$$

Using this expression, we write $W$ as

$$
W=\frac{1}{2 \pi i} f_{-\infty}^{\infty} \frac{\gamma\left(\alpha^{\prime}\right)}{z(\alpha)-z\left(\alpha^{\prime}\right)} d \alpha^{\prime}=\frac{1}{2 i z_{\alpha}} \mathcal{H}[\gamma]+\int_{-\infty}^{\infty} \gamma\left(\alpha^{\prime}\right) g\left(\alpha, \alpha^{\prime}\right) d \alpha^{\prime}
$$

where $\mathcal{H}$ is the Hilbert transform defined by

$$
\mathcal{H}[f](\alpha)=\frac{1}{\pi} \int_{-\infty}^{\infty} \frac{f\left(\alpha^{\prime}\right)}{\alpha-\alpha^{\prime}} d \alpha^{\prime} .
$$

The last term in (14) is of lower order because $\gamma$ is integrated against the smooth function $g$. Therefore, it follows that [24]

$$
U^{N}(\alpha, t)=\frac{1}{2 i \sigma} \mathcal{H}[\gamma](\alpha, t)+R_{s}[\gamma](\alpha, t),
$$

where $R_{s}$ is a smoothing operator in the sense that if $x(\alpha, t)$ and $y(\alpha, t)$ are real analytic functions of $\alpha$ for $t \leq T$ and $\sigma \neq 0$, then the Fourier transform of $R$, satisfies $\hat{R}_{s}[\gamma]=O\left(e^{-\rho|k|} \hat{\gamma}\right)$ for large wavenumber $|k|$. Here, $\rho>0$ is the width of the strip of analyticity about the real axis. If $x(\alpha, t)$ and $y(\alpha, t)$ are not analytic but $C^{m}$, then $\hat{R}_{s}[\gamma]=O\left(|k|^{-m} \hat{\gamma}\right)$. Thus, at high modes,

$$
\frac{1}{\sigma} U_{\alpha}^{N} \sim \frac{1}{2 \sigma}\left(\frac{1}{\sigma} \mathcal{H}[\gamma]\right)_{\alpha},
$$

where by $\sim$ we mean equivalent modulo a smoothing operator. The evolution equations for $\theta$ and $\gamma$ can now be written in a form that reveals the leading order 
behavior at small scales.

$$
\begin{aligned}
\theta_{t} & =\frac{1}{2 \sigma}\left(\frac{1}{\sigma} \mathcal{H}[\gamma]\right)_{\alpha}+P, \\
\gamma_{t} & =S\left(\frac{\theta_{\alpha}}{\sigma}\right)_{\alpha}+Q,
\end{aligned}
$$

where $P$ is defined as the right hand side of (12) minus the high mode leading term and $Q$ is the right hand side of (13) without the surface tension term.

The leading order terms simplify considerably if $\sigma$ is independent of $\alpha$. This can be achieved by taking the parametrization function $R$ identically equal to one. In this particular case, the system (16)-(17) in Fourier space becomes

$$
\begin{aligned}
& \hat{\theta}_{t}(k)=\frac{1}{2 \sigma^{2}}|k| \hat{\gamma}(k)+\hat{P}(k), \\
& \hat{\gamma}_{t}(k)=-\frac{S}{\sigma} k^{2} \hat{\theta}(k)+\hat{Q}(k),
\end{aligned}
$$

where we have used that $\widehat{\mathcal{H} f}(k)=-i \operatorname{sgn}(k) \hat{f}(k)$. An implicit time discretization of the high mode leading order terms is now trivial to solve. If $R \not \equiv 1$, we can recast the system of equations obtained by a semi-implicit discretization in a form suitable for an efficient iterative method [15, 23].

\section{THE NUMERICAL METHOD}

We may construct a numerical scheme for the interface evolution equations (11)(13) simply by providing rules to approximate the spatial derivatives and the singular integrals. However, just as it has been observed by BHL [7] in the case of a boundary integral method for water waves, straightforward discretizations usually lead to unstable schemes. More precisely, a stable discretization must maintain a very delicate balance among the leading order singular terms of the method. To guarantee this balance, some Fourier filtering has to be applied selectively as part of the discretization. The spectral accuracy does not eliminate the need for numerical filtering. Indeed, the periodicity enforced on the numerical solution leads to aliasing errors that ultimately upset the stability balance.

We present and analyze here a spectrally accurate method for two-fluid interfaces. The analysis is more complicated than that of the water waves scheme studied by BHL. Here, we have a more general and different formulation for the interface dynamics.

Let us define first a discrete Fourier transform of a periodic function $f$ whose values are known on a uniform grid of mesh size $h=2 \pi / N$ by

$$
\hat{f}_{k}=\frac{1}{N} \sum_{j=-N / 2+1}^{N / 2} f\left(\alpha_{j}\right) e^{-i k \alpha_{j}} ; \quad \alpha_{j}=j h,
$$

with inverse or Fourier interpolant given by

$$
f\left(\alpha_{j}\right)=\sum_{k=-N / 2+1}^{N / 2} \hat{f}_{k} e^{i k \alpha_{j}} .
$$


Here we assume that $N$ is even. Our numerical filtering is defined in Fourier space as follows:

$$
\widehat{\left(f^{p}\right)_{k}}=\rho(k h) \hat{f}_{k},
$$

where $\rho$ is a cutoff function with the following properties

$$
\begin{aligned}
\rho(-x) & =\rho(x) ; \rho(x) \geq 0, \\
\rho(x) & \in C^{r} ; r>2, \\
\rho( \pm \pi) & =\rho^{\prime}( \pm \pi)=0, \\
\rho(x) & =1 \quad \text { for }|x| \leq \lambda \pi \quad 0<\lambda<1 .
\end{aligned}
$$

Condition (iv) ensures the spectral accuracy of the filtering. While the selective use of filtering in our method prevents high mode instability, it introduces new technical difficulties into the analysis; the commutator of the filtering $\left(f(\alpha) \phi_{i}\right)^{p}-f(\alpha) \phi_{i}^{p}$, for regular $f$, is not smooth enough to be absorbed into low order terms and its particular form, which depends on the properties of $\rho$ must be taken into account in the energy estimates.

We compute the spatial derivatives with a pseudo-spectral approximation, which we denote by $\mathrm{S}_{\mathrm{h}}$, and with a combination of this approximation and our Fourier filtering. This combination is denoted by $\mathrm{D}_{\mathrm{h}}$, that is

$$
\widehat{\left(\mathrm{S}_{\mathrm{h}} f\right)_{k}}=i k \hat{f}_{k} \quad \text { and } \quad \mathrm{D}_{\mathrm{h}} f_{i}=\mathrm{S}_{\mathrm{h}} f_{i}^{p} .
$$

Hereafter, we denote by $\sigma\left(\alpha_{i}\right), \theta\left(\alpha_{i}\right), \gamma\left(\alpha_{i}\right)$, etc., the exact continuous solution evaluated at the grid points, and by $\sigma_{i}, \theta_{i}, \gamma_{i}$, etc., the discrete approximation. To simplify the notation we omit writing the time dependence on the functions unless otherwise noted.

Another difficulty is introduced by the parametrization, and special care must be taken in discretizing $\gamma$ to compute the velocity $W$. We approximate $\gamma\left(\alpha_{j}\right)$ by $\tilde{\gamma}_{j}^{p} \sigma_{j}$ where $\tilde{\gamma}_{j}=\gamma_{j} / \sigma_{j}$, and use the alternate-point trapezoidal rule,

$$
W_{i}=\frac{1}{4 \pi i} \sum_{\substack{j=-N / 2+1 \\(j-i) \text { odd }}}^{N / 2} \tilde{\gamma}_{j}^{p} \sigma_{j} \cot \frac{1}{2}\left(z_{i}^{p}-z_{j}^{p}\right) 2 h .
$$

As we will see later, using $\gamma_{j}^{p}$ instead of $\tilde{\gamma}_{j}^{p} \sigma_{j}$ would introduce a term that may lead to instability.

Sidi and Israeli [39] showed that the alternate-point trapezoidal rule applied to periodic Cauchy kernels such as those considered here, gives spectral accuracy. This quadrature has been used extensively in vortex sheet computations $[4,7,26,38]$.

We still have to provide a discretization for the map (8) to determine the complex position variable $z$ in terms of $\sigma$ and $\theta$. We do this with the following approximation,

$$
z_{i}=\alpha_{i}+\operatorname{Int}_{\mathrm{h}}\left(\sigma e^{i \theta}-\left\langle\sigma e^{i \theta}\right\rangle_{h}\right)_{i},
$$

where Int $_{\mathrm{h}}$ is the pseudo-spectral integral operator defined in Fourier space by

$$
\left.\widehat{\left(\operatorname{Int}_{\mathrm{h}} f\right.}\right)_{k}= \begin{cases}\frac{1}{i k} \hat{f}_{k} & \text { for } k \neq 0 \\ 0 & \text { for } k=0\end{cases}
$$


for any $2 \pi$-periodic function $f$ of mean zero. The discrete mean is computed using the trapezoidal rule,

$$
\langle f\rangle_{h} \equiv \frac{1}{N} \sum_{i=-N / 2+1}^{N / 2} f_{i} .
$$

Note that, without loss of generality, we have also set to zero the integration constant $z_{0}$.

The discretizations for the tangential and normal velocities come from the the approximation (21) for $W$,

$$
U_{i}^{N}=-\operatorname{Im}\left\{e^{i \theta_{i}^{p}} W_{i}\right\} \quad \text { and } \quad U_{i}^{T}=\operatorname{Re}\left\{e^{i \theta_{i}^{p}} W_{i}\right\} .
$$

Now, recall that the parametrization of the interface is determined by our choice (10) of the added tangential velocity $U^{A}$. This velocity is calculated using the next discretization,

$$
U_{i}^{A}=-U_{i}^{T}+\operatorname{Int}_{\mathrm{h}}\left(\mathrm{D}_{\mathrm{h}} \theta U^{N}-R\left\langle\mathrm{D}_{\mathrm{h}} \theta U^{N}\right\rangle_{h}\right)_{i} .
$$

Our method may be written as follows

$$
\begin{aligned}
\frac{d \sigma_{i}}{d t} & =-R\left(\alpha_{i}\right)\left\langle\mathrm{S}_{\mathrm{h}} \theta U^{N}\right\rangle_{h}, \\
\frac{d \theta_{i}}{d t} & =\frac{1}{\sigma_{i}}\left[\mathrm{~S}_{\mathrm{h}} U_{i}^{N}+\mathrm{D}_{\mathrm{h}} \theta_{i}\left(U_{i}^{T}+U_{i}^{A}\right)\right], \\
\frac{d \gamma_{i}}{d t} & =S \mathrm{D}_{\mathrm{h}}\left(\frac{1}{\sigma_{i}} \mathrm{D}_{\mathrm{h}} \theta_{i}\right)+\mathrm{D}_{\mathrm{h}}\left(U_{i}^{A} \tilde{\gamma}_{i}\right)+F_{i} \\
& -2 A\left[\frac{1}{8} \mathrm{D}_{\mathrm{h}} \tilde{\gamma}_{i}^{2}+g \sigma_{i} \sin \theta_{i}-U^{A} \operatorname{Re}\left\{e^{i \theta_{i}} \mathrm{~S}_{\mathrm{h}} W_{i}\right\}\right],
\end{aligned}
$$

where the term $F_{i}$ in (27) is an approximation to $-2 A R e\left\{\sigma e^{i \theta} W_{t}\right\}$ which we split into two terms

$$
\begin{aligned}
F_{i} & =-2 A R e\left\{\frac{\sigma_{i} e^{i \theta_{i}}}{4 \pi i} \sum_{\substack{j=-N / 2+1 \\
(j-i) \text { odd }}}^{N / 2} \frac{d \gamma_{j}}{d t} \cot \frac{1}{2}\left(z_{i}^{p}-z_{j}^{p}\right) 2 h\right\} \\
& +A R e\left\{\frac{\sigma_{i} e^{i \theta_{i}}}{4 \pi i} \sum_{\substack{j=-N / 2+1 \\
(j-i) \text { odd }}}^{N / 2} \gamma_{j}^{p} \frac{\frac{d z_{i}}{d t}-\frac{d z_{j}}{d t}}{\sin ^{2} \frac{1}{2}\left(z_{i}^{p}-z_{j}^{p}\right)} 2 h\right\} .
\end{aligned}
$$

Note that $F_{i}$ is not obtained directly by differentiating with respect to time our approximation (21) for $W$. If such a discretization were used instead, it would introduce explicit filtering to the dependent variable $d \gamma_{i} / d t$. This is both unnecessary and unsatisfactory. It should be noted here that, unless $A=0$, the expression (27) is a discrete version of an integral equation for $d \gamma_{i} / d t$. Later we will show that this discrete linear system for $d \gamma_{i} / d t$ is invertible. Therefore, $d \gamma_{i} / d t$ can be expressed in terms of other variables that have been filtered. As a result, $d \gamma_{i} / d t$ has the filtering effect implicitly. On the other hand, we do need filtering on $\gamma$ in the term that comes from the derivative of the kernel (second term in (21)) to avoid high mode instability. This is quite different from the case for the water wave problem where we do not need an evolution equation for $\gamma$. 
The derivative of $z$ with respect to time in the second term of (28) is obtained from (22), that is

$$
\frac{d z_{i}}{d t}=\operatorname{Int}_{\mathrm{h}}\left(\frac{d \sigma}{d t} e^{i \theta}+i \sigma e^{i \theta} \frac{d \theta}{d t}-\left\langle\frac{d \sigma}{d t} e^{i \theta}+i \sigma e^{i \theta} \frac{d \theta}{d t}\right\rangle_{h}\right)_{i},
$$

where $d \sigma / d t$ and $d \theta / d t$ are the right hand sides of (25) and (26), respectively.

As we will see in the analysis, it is crucial for numerical stability that the leading order terms of $\mathrm{S}_{\mathrm{h}} U_{i}^{N} / \sigma_{i}$ must balance the surface tension term $S \mathrm{D}_{\mathrm{h}}\left(\mathrm{D}_{\mathrm{h}} \theta_{i} / \sigma_{i}\right)$ and a term that comes from the derivative of the added tangential velocity in $\mathrm{D}_{\mathrm{h}}\left(U_{i}^{A} \tilde{\gamma}_{i}\right)$. The use of $\tilde{\gamma}_{j}^{p} \sigma_{j}$ in computing the velocity, the properties of the main part of the quadrature for the velocity (a form of a discrete Hilbert transform), and the relation of its derivative with the pseudo-spectral derivatives of our discretization (Lemma 2 , below) are the key to achieve this delicate balance in the energy estimates. At the same time, special care must be taken not to introduce high mode errors; this is the role of our Fourier filtering. The importance of the filtering will become clear when extracting the leading order terms of the discretization.

We state now a convergence theorem for this numerical method.

Theorem 1. Assume that for $0 \leq t \leq T$ there exist a regular solution of the evolution equations of the interface (11)-(13) with $\sigma(\cdot, t), \theta(\cdot, t)$ and $\gamma(\cdot, t)$ belonging to $C^{m+3}[-\pi, \pi]$ for $m>4$ and that $|z(\alpha, t)-z(\beta, t)| \geq C|\alpha-\beta|$ holds for some $C>0$. If $\sigma_{h}, \theta_{h}$ and $\gamma_{h}$ denote the numerical solution of (25)-(27), then for $h \leq h_{0}(T)$ we have,

$$
\begin{aligned}
& \left\|\sigma_{h}(t)-\sigma(\cdot, t)\right\|_{l^{2}} \leq C(T) h^{m}, \\
& \left\|\theta_{h}(t)-\theta(\cdot, t)\right\|_{H_{h}^{1 / 2}} \leq C(T) h^{m}, \\
& \left\|\gamma_{h}(t)-\gamma(\cdot, t)\right\|_{l^{2}} \leq C(T) h^{m},
\end{aligned}
$$

where

$$
\begin{aligned}
\|u\|_{l^{2}}^{2} & =\sum_{j=-N / 2+1}^{N / 2}\left|u_{j}\right|^{2} h, \\
\|u\|_{H_{h}^{1 / 2}}^{2} & =2 \pi \sum_{k=-N / 2+1}^{N / 2}(1+\rho(k h)|k|)\left|\hat{u}_{k}\right|^{2} .
\end{aligned}
$$

To be consistent with the hypothesis on the regularity of $\sigma(\cdot, t)$, we assume that the parametrization function $R$ is a given $C^{m+3}[-\pi, \pi]$ function of mean one.

The proof of this result is divided in two main parts: Consistency and Stability. The latter is the most technical part of the proof and it is divided itself into three subsections.

\section{Consistency}

We examine next how accurately the exact solution satisfies the discrete system of equations. First note the spectral accuracy of the pseudo-spectral operators (see, e.g., [43]). If $f(\alpha)$ is a periodic $C^{s+1}[-\pi, \pi]$ function, then

$$
\begin{aligned}
\left|\mathrm{S}_{\mathrm{h}} f\left(\alpha_{i}\right)-f_{\alpha}\left(\alpha_{i}\right)\right| & \leq C h^{s}\|f\|_{H^{s+1}}, \\
\left|\mathrm{D}_{\mathrm{h}} f\left(\alpha_{i}\right)-f_{\alpha}\left(\alpha_{i}\right)\right| & \leq C h^{s}\|f\|_{H^{s+1}},
\end{aligned}
$$


and for our Fourier smoothing we have that

$$
\left|f^{p}\left(\alpha_{i}\right)-f\left(\alpha_{i}\right)\right| \leq C h^{s+1}\|f\|_{H^{s+1}} .
$$

It is well known that the trapezoidal quadrature rule, when applied to periodic functions gives spectral accuracy [22], that is,

$$
\left|\sum_{j=-N / 2+1}^{N / 2} f\left(\alpha_{j}\right) h-\int_{-\pi}^{\pi} f(\alpha) d \alpha\right| \leq C h^{s+1}\|f\|_{H^{s+1}} .
$$

Similarly for the pseudo-spectral integral operator, we have

$$
\left|\operatorname{Int}_{\mathrm{h}}(g(\cdot))_{i}-\int_{0}^{\alpha_{i}} g(\alpha) d \alpha\right| \leq C h^{s+1}\|g\|_{H^{s}},
$$

for zero-mean and periodic $g(\alpha) \in C^{s}[-\pi, \pi]$. Thus, from these observations and from the assumption on the regularity of the exact solution, it follows that

$$
\begin{aligned}
z_{h}\left(\alpha_{i}\right) & \equiv \alpha_{i}+\operatorname{Int}_{\mathrm{h}}\left(\sigma(\cdot) e^{i \theta(\cdot)}-\left\langle\sigma e^{i \theta}\right\rangle_{h}\right)_{i} \\
& =\alpha_{i}+\int_{0}^{\alpha_{i}}\left(\sigma e^{i \theta}-1\right) d \alpha+O\left(h^{m+3}\right)=z\left(\alpha_{i}\right)+O\left(h^{m+3}\right),
\end{aligned}
$$

where we have also used that $\left\langle z_{\alpha}(\cdot)\right\rangle=1$.

Let us denote by $W_{h}\left(\alpha_{i}\right), U_{h}^{T}\left(\alpha_{i}\right), U_{h}^{A}\left(\alpha_{i}\right)$, and $U_{h}^{N}\left(\alpha_{i}\right)$ the velocities obtained by substituting the exact solution $\sigma(\cdot, t), \theta(\cdot, t)$ and $\gamma(\cdot, t)$ into the discrete equations (21)-(24), to distinguish them from the corresponding exact velocities. In order to examine the accuracy of the approximation for the velocity, we note that

$$
\tilde{\gamma}^{p}\left(\alpha_{j}\right)=\tilde{\gamma}\left(\alpha_{j}\right)+O\left(h^{m+3}\right)=\frac{\gamma\left(\alpha_{j}\right)}{\sigma\left(\alpha_{j}\right)}+O\left(h^{m+3}\right) .
$$

Thus, using the spectral accuracy of the smoothing and (35), we get

$$
\tilde{\gamma}^{p}\left(\alpha_{j}\right) \sigma\left(\alpha_{j}\right) \cot \frac{1}{2}\left(z_{h}^{p}\left(\alpha_{i}\right)-z_{h}^{p}\left(\alpha_{j}\right)\right)=\gamma\left(\alpha_{j}\right) \cot \frac{1}{2}\left(z\left(\alpha_{i}\right)-z\left(\alpha_{j}\right)\right)+O\left(h^{m+3}\right) .
$$

It was shown in $[7,38,39]$ that the alternate-point trapezoidal rule applied to this integrand yields spectral accuracy, that is

$$
\begin{aligned}
& \sum_{\substack{j=-N / 2+1 \\
(j-i) \text { odd }}}^{N / 2} \gamma\left(\alpha_{j}\right) \cot \frac{1}{2}\left(z^{p}\left(\alpha_{i}\right)-z^{p}\left(\alpha_{j}\right)\right) 2 h \\
& =\int_{-\pi}^{\pi} \gamma\left(\alpha^{\prime}\right) \cot \frac{1}{2}\left(z\left(\alpha_{i}\right)-z\left(\alpha^{\prime}\right)\right) d \alpha^{\prime}+O\left(h^{m+2}\right) .
\end{aligned}
$$

Therefore, for our approximation to the complex velocity $W$, we have

$$
\begin{aligned}
W_{h}\left(\alpha_{i}\right) & \equiv \frac{1}{4 \pi i} \sum_{\substack{j=-N / 2+1 \\
(j-i) \text { odd }}}^{N / 2} \tilde{\gamma}^{p}\left(\alpha_{j}\right) \sigma\left(\alpha_{j}\right) \cot \frac{1}{2}\left(z^{p}\left(\alpha_{i}\right)-z^{p}\left(\alpha_{j}\right)\right) 2 h \\
& =W\left(\alpha_{i}\right)+O\left(h^{m+2}\right) .
\end{aligned}
$$

Note that since $\theta^{p}\left(\alpha_{i}\right)=\theta\left(\alpha_{i}\right)+O\left(h^{m+3}\right)$, it now follows that

$$
\begin{gathered}
U_{h}^{N}\left(\alpha_{i}\right)=U^{N}\left(\alpha_{i}\right)+O\left(h^{m+2}\right), \\
U_{h}^{T}\left(\alpha_{i}\right)=U^{T}\left(\alpha_{i}\right)+O\left(h^{m+2}\right),
\end{gathered}
$$


and using (31)-(34), we also have that

$$
U_{h}^{A}\left(\alpha_{i}\right)=U^{A}\left(\alpha_{i}\right)+O\left(h^{m+2}\right) .
$$

It is now easy to check the consistency for the $\sigma$-equation (25), because

$$
\begin{aligned}
\left\langle\mathrm{S}_{\mathrm{h}} \theta(\cdot) U_{h}^{N}(\cdot)\right\rangle_{h} & =\left\langle\theta_{\alpha}(\cdot) U^{N}(\cdot)\right\rangle_{h}+O\left(h^{m+2}\right) \\
& =\left\langle\theta_{\alpha}(\cdot) U^{N}(\cdot)\right\rangle+O\left(h^{m+2}\right)
\end{aligned}
$$

and consequently

$$
\frac{d \sigma}{d t}\left(\alpha_{i}\right)=-R\left(\alpha_{i}\right)\left\langle\mathrm{S}_{\mathrm{h}} \theta(\cdot) U_{h}^{N}(\cdot)\right\rangle_{h}+O\left(h^{m+2}\right) .
$$

Similarly, for the $\theta$-equation (26) we obtain

$$
\frac{d \theta}{d t}\left(\alpha_{i}\right)=\frac{1}{\sigma\left(\alpha_{i}\right)}\left[\mathrm{S}_{\mathrm{h}} U_{h}^{N}\left(\alpha_{i}\right)+\mathrm{D}_{\mathrm{h}} \theta\left(\alpha_{i}\right)\left(U^{T}\left(\alpha_{i}\right)+U^{A}\left(\alpha_{i}\right)\right)\right]+O\left(h^{m+1}\right) .
$$

Now from (29), using (33) and (34), it follows that

$$
\frac{d z_{h}}{d t}\left(\alpha_{i}\right)=\frac{d z}{d t}\left(\alpha_{i}\right)+O\left(h^{m+2}\right)
$$

Note also that we may write

$$
\begin{aligned}
& \sum_{\substack{j=-N / 2+1 \\
(j-i) \text { odd }}}^{N / 2} \gamma\left(\alpha_{j}\right)^{p} \frac{\frac{d z_{h}}{d t}\left(\alpha_{i}\right)-\frac{d z_{h}}{d t}\left(\alpha_{j}\right)}{\sin ^{2} \frac{1}{2}\left(z_{h}^{p}\left(\alpha_{i}\right)-z_{h}^{p}\left(\alpha_{j}\right)\right)} 2 h \\
& \quad=\sum_{\substack{j=-N / 2+1 \\
(j-i) \text { odd }}}^{N / 2} G\left(\alpha_{i}, \alpha_{j}\right) \cot \frac{1}{2}\left(z\left(\alpha_{i}\right)-z\left(\alpha_{j}\right)\right) 2 h+O\left(h^{m+2}\right),
\end{aligned}
$$

where

$$
G\left(\alpha_{i}, \alpha_{j}\right) \equiv 2 \gamma\left(\alpha_{j}\right) \frac{\frac{d z}{d t}\left(\alpha_{i}\right)-\frac{d z}{d t}\left(\alpha_{j}\right)}{\sin \left(z\left(\alpha_{i}\right)-z\left(\alpha_{j}\right)\right)} .
$$

Hence $G(\cdot, \cdot) \in C^{m+1}[-\pi, \pi]$ (it has a removable singularity) and, as a result, the alternate point trapezoidal rule yields an $O\left(h^{m}\right)$ approximation for this integral. On the other hand, in view of (31), we have that

$$
\mathrm{D}_{\mathrm{h}}\left(\frac{1}{\sigma\left(\alpha_{i}\right)} \mathrm{D}_{\mathrm{h}} \theta\left(\alpha_{i}\right)\right)=\left(\frac{1}{\sigma\left(\alpha_{i}\right)} \theta_{\alpha}\left(\alpha_{i}\right)\right)_{\alpha}+O\left(h^{m}\right)
$$

and similarly for the other terms of the $\gamma$-equation (27). The following lemma summarizes the accuracy of the numerical method.

Consistency Lemma. The exact solution of the interface equations (11)-(13) satisfies the discrete evolution system (25)-(27) with truncation error of order $O\left(h^{m+2}\right)$ for equation (25), of order $O\left(h^{m+1}\right)$ for equation (26), and of order $O\left(h^{m}\right)$ for equation (27). 


\section{Stability}

Let us define the errors or variations between the exact and numerical solutions as follows,

$$
\begin{gathered}
\dot{\sigma}_{j} \equiv \sigma_{j}-\sigma\left(\alpha_{j}\right), \\
\dot{\theta}_{j} \equiv \theta_{j}-\theta\left(\alpha_{j}\right), \\
\dot{\tilde{\gamma}}_{j} \equiv \tilde{\gamma}_{j}-\tilde{\gamma}\left(\alpha_{j}\right) .
\end{gathered}
$$

Our goal is to obtain a system of evolution equations for these errors and perform energy estimates to show they remain bounded in suitable discrete norms for $t \leq T$. Recall we are assuming that a smooth exact solution exists up to time $T$. To illustrate the idea, let us find an equation for $\dot{\theta}$. First, substitute the exact solution into (26) and use consistency. Then, substract the resulting equation to (26) and get

$$
\begin{aligned}
\frac{d \dot{\theta}_{i}}{d t}= & \frac{1}{\sigma_{i}}\left[\mathrm{~S}_{\mathrm{h}} U_{i}^{N}+\mathrm{D}_{\mathrm{h}} \theta_{i}\left(U_{i}^{T}+U_{i}^{A}\right)\right] \\
& -\frac{1}{\sigma\left(\alpha_{i}\right)}\left[\mathrm{S}_{\mathrm{h}} U_{h}^{N}\left(\alpha_{i}\right)+\mathrm{D}_{\mathrm{h}} \theta\left(\alpha_{i}\right)\left(U_{h}^{T}\left(\alpha_{i}\right)+U_{h}^{A}\left(\alpha_{i}\right)\right)\right]+O\left(h^{m+1}\right) .
\end{aligned}
$$

Now, the right hand side of this equation can be written in terms of $\dot{\sigma}_{i}, \dot{\theta}_{i}$, and the variations in the velocities,

$$
\begin{aligned}
\dot{U}_{i}^{N} & \equiv U_{i}^{N}-U_{h}^{N}\left(\alpha_{i}\right), \\
\dot{U}_{i}^{T} & \equiv U_{i}^{T}-U_{h}^{T}\left(\alpha_{i}\right), \\
\dot{U}_{i}^{A} & \equiv U_{i}^{A}-U_{h}^{A}\left(\alpha_{i}\right) .
\end{aligned}
$$

Therefore, our first task is to estimate $\dot{U}_{i}^{N}, \dot{U}_{i}^{T}$, and $\dot{U}_{i}^{A}$ in terms of the errors $\dot{\sigma}_{i}, \dot{\theta}_{i}$ and $\dot{\tilde{\gamma}}_{i}$. This is accomplished by identifying the most singular part in the variation $\dot{W}_{i}=W_{i}-W_{h}\left(\alpha_{i}\right)$ of the complex velocity. The estimates separate into linear and nonlinear terms in $\dot{\sigma}_{i}, \dot{\theta}_{i}$ and $\dot{\tilde{\gamma}}_{i}$. The nonlinear terms can be controlled because of the high accuracy of the method for smooth solutions. Thus, the leading order contribution comes from the linear terms.

The following notation will help us handle low order terms arising in the stability analysis. We define a discrete $s$-th order smoothing operator, written generically $A_{-s}$, as an operator satisfying

$$
\left\|\mathrm{D}_{\mathrm{h}}{ }^{l}\left(A_{-s}(\dot{\phi})\right)\right\|_{l^{2}} \leq C\|\dot{\phi}\|_{l^{2}} \quad \text { and } \quad\left\|A_{-s}\left(\mathrm{~S}_{\mathrm{h}}{ }^{l} \dot{\phi}\right)\right\|_{l^{2}} \leq C\|\dot{\phi}\|_{l^{2}} \quad \text { for } \quad 0 \leq l \leq s .
$$

Note in particular that $A_{0}\left(\dot{\phi}_{i}\right)$ is a $l^{2}$-bounded operator, that is, $\left\|A_{0}(\dot{\phi})\right\|_{l^{2}} \leq$ $C\|\dot{\phi}\|_{l^{2}}$. Moreover, it is easy to see that $h^{s} A_{0}\left(\dot{\phi}_{i}\right)=A_{-s}\left(\dot{\phi}_{i}\right)$. We also write

$$
A_{-s}\left(\dot{\phi}_{i}, \dot{\psi}_{i}, \ldots, \dot{\eta}_{i}\right) \equiv A_{-s}\left(\dot{\phi}_{i}\right)+A_{-s}\left(\dot{\psi}_{i}\right)+\cdots+A_{-s}\left(\dot{\eta}_{i}\right) \text {. }
$$

5.1. Estimates for the Variations of the Velocities. As noted by BHL [7], in estimating the variation in the complex velocity, it is easier to work with the kernel defined on the entire line rather than in $[-\pi, \pi]$. The periodic extension is a straightforward application of the formula,

$$
\frac{1}{2} \cot \frac{1}{2} z=\frac{1}{z}+\sum_{k=1}^{\infty} \frac{2 z}{z^{2}-(2 k \pi)^{2}}
$$


which converges uniformly in any compact set not containing the points $z=2 k \pi$, with $k$ integer [31]. We obtain (see [7, 15] for details)

$$
\begin{aligned}
\frac{1}{2} & \sum_{\substack{j=-N / 2+1 \\
(j-i) \text { odd }}}^{N / 2} f\left(\alpha_{j}\right) \cot \frac{1}{2}\left(z\left(\alpha_{i}\right)-z\left(\alpha_{j}\right)\right) 2 h \\
& =\lim _{M \rightarrow \infty} \sum_{\substack{j=-N(M+1 / 2)+1 \\
(j-i) \text { odd }}}^{N(M+1 / 2)} \frac{f\left(\alpha_{j}\right)}{z\left(\alpha_{i}\right)-z\left(\alpha_{j}\right)} 2 h .
\end{aligned}
$$

To simplify the notation, we define

$$
\sum_{(j-i) \text { odd }} \frac{f\left(\alpha_{j}\right)}{z\left(\alpha_{i}\right)-z\left(\alpha_{j}\right)} 2 h \equiv \lim _{M \rightarrow \infty} \sum_{\substack{j=-N(M+1 / 2)+1 \\(j-i) \text { odd }}}^{N(M+1 / 2)} \frac{f\left(\alpha_{j}\right)}{z\left(\alpha_{i}\right)-z\left(\alpha_{j}\right)} 2 h .
$$

Analogously, a periodic extension of the numerical solution yields,

$$
\sum_{\substack{j=-N / 2+1 \\(j-i) \text { odd }}}^{N / 2} f_{j} \cot \frac{1}{2}\left(z_{i}-z_{j}\right) 2 h=\sum_{(j-i) \text { odd }} \frac{f_{j}}{z_{i}-z_{j}} 2 h .
$$

It is also convenient to extend the kernel $1 / \sin ^{2} z$ that appears in one of the terms of (28). In this case we get,

$$
\sum_{\substack{j=-N / 2+1 \\(j-i) \text { odd }}}^{N / 2} \gamma_{j}^{p} \frac{\frac{d z_{i}}{d t}-\frac{d z_{j}}{d t}}{\sin ^{2} \frac{1}{2}\left(z_{i}^{p}-z_{j}^{p}\right)} 2 h=\sum_{(j-i) \text { odd }} \gamma_{j}^{p} \frac{\frac{d z_{i}}{d t}-\frac{d z_{j}}{d t}}{\left(z_{i}^{p}-z_{j}^{p}\right)^{2}} 2 h .
$$

We devote the main part of this subsection to show that

$$
\dot{W}_{i}=\frac{\sigma\left(\alpha_{i}\right)}{2 i z_{\alpha}(\alpha)} \mathrm{H}_{\mathrm{h}} \dot{\tilde{\gamma}}_{i}^{p}-\frac{\gamma\left(\alpha_{i}\right)}{2 z_{\alpha}\left(\alpha_{i}\right)} \mathrm{H}_{\mathrm{h}} \dot{\theta}_{i}^{p}+\cdots,
$$

where $\mathrm{H}_{\mathrm{h}}$ is a discrete Hilbert transform given by

$$
\mathrm{H}_{\mathrm{h}} f_{i} \equiv \frac{1}{\pi} \sum_{(j-i) \text { odd }} \frac{f_{j}}{\alpha_{i}-\alpha_{j}} 2 h .
$$

Thus, the particular combination of $\mathrm{H}_{\mathrm{h}}$ in (38), extracts the most singular part of $\dot{W}_{i}$; the terms that we are omitting are of lower order. To obtain this estimate, we follow BHL [7] and write $\dot{W}_{i}$ as a sum of linear and nonlinear terms in the errors $\dot{\sigma}, \dot{\tilde{\gamma}}$, and $\dot{z}$ as follows

$$
\begin{aligned}
\dot{W}_{i} & =\frac{1}{2 \pi i} \sum_{(j-i) \text { odd }} \frac{\dot{\sigma}_{j} \tilde{\gamma}^{p}\left(\alpha_{j}\right)}{z_{h}^{p}\left(\alpha_{i}\right)-z_{h}^{p}\left(\alpha_{j}\right)} 2 h+\frac{1}{2 \pi i} \sum_{(j-i) \text { odd }} \frac{\sigma\left(\alpha_{j}\right) \dot{\tilde{\gamma}}_{j}^{p}}{z_{h}^{p}\left(\alpha_{i}\right)-z_{h}^{p}\left(\alpha_{j}\right)} 2 h \\
& -\frac{1}{2 \pi i} \sum_{(j-i) \text { odd }} \sigma\left(\alpha_{j}\right) \tilde{\gamma}^{p}\left(\alpha_{j}\right) \frac{\dot{z}_{i}^{p}-\dot{z}_{j}^{p}}{\left(z_{h}^{p}\left(\alpha_{i}\right)-z_{h}^{p}\left(\alpha_{j}\right)\right)^{2}} 2 h+\dot{W}_{i}^{N L},
\end{aligned}
$$


where $\dot{W}_{i}^{N L}$ stands for the nonlinear part given by

$$
\begin{aligned}
\dot{W}_{i}^{N L}= & \frac{1}{2 \pi i} \sum_{(j-i) \text { odd }} \frac{\dot{\sigma}_{j} \dot{\tilde{\gamma}}_{j}^{p}}{z_{h}^{p}\left(\alpha_{i}\right)-z_{h}^{p}\left(\alpha_{j}\right)} 2 h \\
& +\frac{1}{2 \pi i} \sum_{(j-i) \text { odd }} \frac{\left(\sigma \dot{\gamma}^{p}\right)_{j}\left(\dot{z}_{i}^{p}-\dot{z}_{j}^{p}\right)}{\left(z_{h}^{p}\left(\alpha_{i}\right)-z_{h}^{p}\left(\alpha_{j}\right)\right)^{2}} 2 h \\
& -\frac{1}{2 \pi i} \sum_{(j-i) \text { odd }} \frac{\sigma_{j} \tilde{\gamma}_{j}^{p}\left(\dot{z}_{i}^{p}-\dot{z}_{j}^{p}\right)^{2} 2 h}{\left(z_{h}^{p}\left(\alpha_{i}\right)-z_{h}^{p}\left(\alpha_{j}\right)\right)^{2}\left(z_{h}^{p}\left(\alpha_{i}\right)-z_{h}^{p}\left(\alpha_{j}\right)+\dot{z}_{i}^{p}-\dot{z}_{j}^{p}\right)} .
\end{aligned}
$$

Our immediate task now is to estimate $\dot{z}$ in terms of $\dot{\sigma}$ and $\dot{\theta}$, and to establish a bound for the nonlinear terms. To this end, let us define the following time,

$$
T^{*} \equiv \operatorname{Sup}\left\{t \mid t \leq T,\|\dot{\sigma}\|_{l^{2}},\|\dot{\theta}\|_{l^{2}},\|\dot{\gamma}\|_{l^{2}} \leq h^{7 / 2} \text { and }\left\|\frac{d \dot{\gamma}}{d t}\right\|_{l^{2}} \leq h^{3 / 2}\right\} .
$$

Unless otherwise noted, all the estimates we obtain hereafter are valid for $t \leq T^{*}$. At the end, we close this so called $T^{*}$-argument, by showing that $T^{*}=T$.

Note that since

$$
h\left|\dot{\theta}_{i}\right|^{2} \leq \sum_{i=-N / 2+1}^{N / 2}\left|\dot{\theta}_{i}\right|^{2} h=\|\dot{\theta}\|_{l^{2}},
$$

we have that (for $t \leq T^{*}$ ),

$$
\|\dot{\theta}\|_{\infty} \leq h^{-1 / 2}\|\dot{\theta}\|_{l^{2}} \leq h^{3}
$$

and similarly,

$$
\|\dot{\sigma}\|_{\infty} \leq h^{3} \quad \text { and } \quad\|\dot{\tilde{\gamma}}\|_{\infty} \leq h^{3} .
$$

On the other hand, the variation $\dot{z}_{i} \equiv z_{i}-z_{h}\left(\alpha_{i}\right)$ is given by

$$
\dot{z}_{i}=\operatorname{Int}_{\mathrm{h}}\left[\left(\sigma e^{i \theta}\right)_{i}^{\cdot}-\left\langle\left(\sigma e^{i \theta}\right)_{i}^{\cdot}\right\rangle_{h}\right],
$$

where

$$
\left(\sigma e^{i \theta}\right)_{i}^{\cdot} \equiv \sigma_{i} e^{i \theta_{i}}-\sigma\left(\alpha_{i}\right) e^{i \theta\left(\alpha_{i}\right)}=\sigma_{i}\left(e^{i \theta_{i}}-e^{i \theta\left(\alpha_{i}\right)}\right)+\dot{\sigma}_{i} e^{i \theta\left(\alpha_{i}\right)} .
$$

Now,

$$
e^{i \theta_{i}}-e^{i \theta\left(\alpha_{i}\right)}=e^{i\left(\dot{\theta}_{i}+\theta\left(\alpha_{i}\right)\right)}-e^{i \theta\left(\alpha_{i}\right)}=i e^{i \theta\left(\alpha_{i}\right)} \dot{\theta}_{i}+r\left(\alpha_{i}\right) \dot{\theta}_{i}^{2}
$$

with $\|r\|_{\infty} \leq 1 / 2$. Moreover, since $\left\|\dot{\theta}^{2}\right\|_{l^{2}} \leq C h^{3}\|\dot{\theta}\|_{l^{2}}$, we get

$$
e^{i \theta_{i}}-e^{i \theta\left(\alpha_{i}\right)}=i e^{i \theta\left(\alpha_{i}\right)} \dot{\theta}_{i}+A_{-3}\left(\dot{\theta}_{i}\right) .
$$

Therefore, using this estimate and noting that $\sigma_{i}=\sigma\left(\alpha_{i}\right)+\dot{\sigma}_{i}$, we get

$$
\left(\sigma e^{i \theta}\right)_{i}=i \sigma\left(\alpha_{i}\right) e^{i \theta\left(\alpha_{i}\right)} \dot{\theta}_{i}+e^{i \theta\left(\alpha_{i}\right)} \dot{\sigma}_{i}+A_{-3}\left(\dot{\theta}_{i}\right),
$$

where we have also used that $\left\|\dot{\sigma}^{2}\right\|_{l^{2}} \leq C h^{3}\|\dot{\sigma}\|_{l^{2}}$ to bound the nonlinear terms. Now, clearly $\langle f(\cdot) \dot{\phi}\rangle_{h}=A_{-s}(\dot{\phi})$ for $f \in C^{s}$. Consequently, substituting (45) into (43) we obtain

$$
\dot{z}_{i}=\operatorname{Int}_{\mathrm{h}}\left(i \sigma(\cdot) e^{i \theta(\cdot)} \dot{\theta}\right)_{i}+\operatorname{Int}_{\mathrm{h}}\left(e^{i \theta(\cdot)} \dot{\sigma}\right)_{i}+A_{-3}\left(\dot{\sigma}_{i}, \dot{\theta}_{i}\right) .
$$


Recall that the frame is selected such that the initial parametrization is preserved up to a time dependent scaling. Thus

$$
\dot{\sigma}_{i}=R\left(\alpha_{i}\right)\left[\langle\sigma\rangle_{h}-\langle\sigma(\cdot)\rangle\right]=R\left(\alpha_{i}\right)\langle\dot{\sigma}\rangle_{h}+O\left(h^{m+3}\right),
$$

where we have also used (33). The relation (46) tells us that the error in $\sigma$ is on the scaling factor $\langle\sigma\rangle_{h}$ only. This time-varying factor may be pulled outside any spacial discrete operator. In particular,

$$
\begin{aligned}
\operatorname{Int}_{\mathrm{h}}(f(\cdot) \dot{\sigma})_{i} & =\langle\dot{\sigma}\rangle_{h} \operatorname{Int}_{\mathrm{h}}(R(\cdot) f(\cdot))_{i}+O\left(h^{m+3}\right) \\
& =\tilde{f}\left(\alpha_{i}\right)\langle\dot{\sigma}\rangle_{h}+O\left(h^{m+3}\right)=A_{-s}\left(\dot{\sigma}_{i}\right)+O\left(h^{m+3}\right),
\end{aligned}
$$

because both the parametrization function $R$ and $f$ are smooth. Similarly we have

$$
\mathrm{D}_{\mathrm{h}}\left(f\left(\alpha_{i}\right) \dot{\sigma}_{i}\right)=g\left(\alpha_{i}\right)\langle\dot{\sigma}\rangle_{h}+O\left(h^{m+2}\right)=A_{-s}\left(\dot{\sigma}_{i}\right)+O\left(h^{m+2}\right)
$$

and the same is true for $\mathrm{S}_{\mathrm{h}}$ instead of $\mathrm{D}_{\mathrm{h}}$. In this sense, terms involving $\dot{\sigma}$ are of lower order. Thus, because of the control we have on $\sigma$, the most singular terms are only those containing $\dot{\theta}$ and $\dot{\tilde{\gamma}}$.

With these observations and noting that $z_{\alpha}(\alpha)=\sigma(\alpha) e^{i \theta(\alpha)}$, we obtain our final estimate for $\dot{z}$,

$$
\dot{z}_{i}=i \operatorname{Int}_{\mathrm{h}}\left(z_{\alpha}(\cdot) \dot{\theta}\right)_{i}+A_{-3}\left(\dot{\sigma}_{i}, \dot{\theta}_{i}\right)+O\left(h^{m+3}\right),
$$

from which we obtain the following bounds

$$
\|\dot{z}\|_{l^{2}} \leq C h^{7 / 2} \text { and }\|\dot{z}\|_{\infty} \leq C h^{3} .
$$

Let us return now to (40), our expression for $\dot{W}$. The first term involves $\dot{\sigma}$. Then, in view of (46), we have

$$
\frac{1}{2 \pi i} \sum_{(j-i) \text { odd }} \frac{\dot{\sigma}_{j} \tilde{\gamma}^{p}\left(\alpha_{j}\right)}{z_{h}^{p}\left(\alpha_{i}\right)-z_{h}^{p}\left(\alpha_{j}\right)} 2 h=A_{-s}\left(\dot{\sigma}_{i}\right)+O\left(h^{m+3}\right) .
$$

Now, to estimate the second term in (40), we first note that

$$
\frac{1}{z(\alpha)-z(\beta)}=\frac{1}{z_{\alpha}(\alpha)(\alpha-\beta)}+g(\alpha, \beta)
$$

where $g(\cdot, \cdot)$ is a smooth function in both variables. On the other hand, by consistency of the approximation,

$$
\frac{1}{z_{h}^{p}\left(\alpha_{i}\right)-z_{h}^{p}\left(\alpha_{j}\right)}=\left(1+O\left(h^{m+3}\right)\right) \frac{1}{z\left(\alpha_{i}\right)-z\left(\alpha_{j}\right)} .
$$

Thus, for the second term in (40), we obtain

$$
\begin{aligned}
& \frac{1}{2 \pi i} \sum_{(j-i) \text { odd }} \frac{\sigma\left(\alpha_{j}\right) \dot{\tilde{\gamma}}_{j}^{p}}{z_{h}^{p}\left(\alpha_{i}\right)-z_{h}^{p}\left(\alpha_{j}\right)} 2 h \\
& \quad=\left(1+O\left(h^{m+3}\right)\right)\left(\frac{1}{2 i z_{\alpha}} \mathrm{H}_{\mathrm{h}}\left(\sigma \dot{\tilde{\gamma}}_{i}^{p}\right)+\sum_{(j-i) \text { odd }} \tilde{g}\left(\alpha_{i}, \alpha_{j}\right) \dot{\tilde{\gamma}}_{j}^{p} 2 h\right) .
\end{aligned}
$$

The term involving the smooth function $\tilde{g}$, is a discrete operator which deserves special care. Let us denote a general operator of this type by $\mathrm{R}_{\mathrm{h}}$, that is

$$
\mathrm{R}_{\mathrm{h}}\left(\phi_{i}\right)=\sum_{(j-i) \text { odd }} g\left(\alpha_{i}, \alpha_{j}\right) \phi_{j} 2 h
$$

for $g(\cdot, \cdot) \in C^{s}$. Clearly the continuous analogue of $\mathrm{R}_{\mathrm{h}}$, is a smoothing operator. However, this is not true at the discrete level, where $\mathrm{R}_{\mathrm{h}}\left(\phi_{i}\right)$ can be as singular as 
$\phi_{i},[11,24]$. We overcome this difficulty with the application of Fourier filtering. Using the properties of the cutoff function $\rho$ which defines our filtering, we obtain the following result proved by BHL [7].

Lemma 1. Let $g(\cdot, \cdot)$ in $(50)$ be in $C^{s}$ with $s>3$, then

$$
\mathrm{R}_{\mathrm{h}}\left(\phi_{i}^{p}\right)=A_{-2}\left(\phi_{i}\right) .
$$

This lemma explains the use of filtering on $\tilde{\gamma}$ when computing $W$. We will see below the reason for performing the smoothing on $\tilde{\gamma}=\gamma / \sigma$ instead of $\gamma$.

Note also that the commutator of the discrete Hilbert transform and a smooth function is an $R_{h}$ operator, that is

$$
\mathrm{H}_{\mathrm{h}}\left(f\left(\alpha_{i}\right) \phi_{i}\right)-f\left(\alpha_{i}\right) \mathrm{H}_{\mathrm{h}}\left(\phi_{i}\right)=\mathrm{R}_{\mathrm{h}}\left(\phi_{i}\right) .
$$

Therefore, we can pull the smooth function $\sigma(\alpha)$ outside $\mathrm{H}_{\mathrm{h}}$ in (49), at the expense of adding a term $\mathrm{R}_{\mathrm{h}}\left(\dot{\tilde{\gamma}}_{i}^{p}\right)=A_{-2}\left(\dot{\tilde{\gamma}}_{i}\right)$. Consequently,

$$
\frac{1}{2 \pi i} \sum_{(j-i) \text { odd }} \frac{\sigma\left(\alpha_{j}\right) \dot{\tilde{\gamma}}_{j}^{p}}{z_{h}^{p}\left(\alpha_{i}\right)-z_{h}^{p}\left(\alpha_{j}\right)} 2 h=\frac{\sigma\left(\alpha_{i}\right)}{2 i z_{\alpha}(\alpha)} \mathrm{H}_{\mathrm{h}}\left(\dot{\tilde{\gamma}}_{i}^{p}\right)+A_{-2}\left(\dot{\tilde{\gamma}}_{i}\right) .
$$

This expression exhibits clearly the leading order contribution of $\dot{\tilde{\gamma}}$ in the variation $\dot{W}$ of the velocity. The dependence on the underlying smooth solution has been absorbed into the coefficient in front of $\mathrm{H}_{\mathrm{h}}$, which is a much simpler operator to deal with. In particular, $\mathrm{H}_{\mathrm{h}}$ satisfies properties similar to those satisfied by the Hilbert transform at the continuous level (15). The following lemma shown by BHL [7] summarizes some of these properties.

Lemma 2. Let $f$ be such that $\hat{f}_{0}=\hat{f}_{N / 2}=0$, then

$$
\begin{aligned}
{\widehat{\left(\mathrm{H}_{\mathrm{h}} f\right.}}_{k} & =-i \operatorname{sgn}(k) \hat{f}_{k}, \\
\mathrm{H}_{\mathrm{h}}\left(\mathrm{H}_{\mathrm{h}} f_{i}\right) & =-f_{i}, \\
\mathrm{H}_{\mathrm{h}}\left(\mathrm{S}_{\mathrm{h}} f_{i}\right) & =\frac{1}{\pi} \sum_{(j-i) \text { odd }} \frac{f_{i}-f_{j}}{\left(\alpha_{i}-\alpha_{j}\right)^{2}} 2 h, \\
\mathrm{~S}_{\mathrm{h}}\left(\mathrm{H}_{\mathrm{h}} f_{i}\right) & =\mathrm{H}_{\mathrm{h}}\left(\mathrm{S}_{\mathrm{h}} f_{i}\right) .
\end{aligned}
$$

To estimate the third term in (40), we extract the singular parts of the kernel as follows,

$$
\frac{\gamma(\beta)}{(z(\alpha)-z(\beta))^{2}}=\frac{\gamma(\alpha)}{z_{\alpha}^{2}(\alpha)(\alpha-\beta)^{2}}+\frac{p(\alpha)}{\alpha-\beta}+q(\alpha, \beta),
$$

where $p(\cdot)$ and $q(\cdot, \cdot)$ are smooth functions. Again, by consistency, we also have that

$$
\frac{\sigma\left(\alpha_{j}\right) \tilde{\gamma}^{p}\left(\alpha_{j}\right)}{\left(z^{p}\left(\alpha_{i}\right)-z^{p}\left(\alpha_{j}\right)\right)^{2}}=\left(1+O\left(h^{m+2}\right)\right) \frac{\gamma\left(\alpha_{j}\right)}{\left(z\left(\alpha_{i}\right)-z\left(\alpha_{j}\right)\right)^{2}} .
$$

Combining the last two expressions and using part (iii) of Lemma 2, and Lemma 1, we obtain

$$
\begin{aligned}
& \frac{1}{2 \pi i} \sum_{(j-i) \text { odd }} \sigma\left(\alpha_{j}\right) \tilde{\gamma}^{p}\left(\alpha_{j}\right) \frac{\dot{z}_{i}^{p}-\dot{z}_{j}^{p}}{\left(z_{h}^{p}\left(\alpha_{i}\right)-z_{h}^{p}\left(\alpha_{j}\right)\right)^{2}} 2 h \\
& \quad=-\frac{\gamma\left(\alpha_{i}\right)}{2 i z_{\alpha}^{2}\left(\alpha_{i}\right)} \mathrm{H}_{\mathrm{h}} \mathrm{D}_{\mathrm{h}} \dot{z}_{i}+f\left(\alpha_{i}\right) \mathrm{H}_{\mathrm{h}} \dot{z}_{i}^{p}+g\left(\alpha_{i}\right) \dot{z}_{i}^{p}+A_{-2}\left(\dot{z}_{i}\right) .
\end{aligned}
$$


We substitute now our estimate (47) into the term containing $\dot{z}$,

$$
-\frac{\gamma\left(\alpha_{i}\right)}{2 i z_{\alpha}^{2}\left(\alpha_{i}\right)} \mathrm{H}_{\mathrm{h}} \mathrm{D}_{\mathrm{h}} \dot{z}_{i}=-\frac{\gamma\left(\alpha_{i}\right)}{2 z_{\alpha}^{2}\left(\alpha_{i}\right)} \mathrm{H}_{\mathrm{h}}\left(z_{\alpha} \dot{\theta}\right)_{i}^{p}+A_{-2}\left(\dot{\sigma}_{i}, \dot{\theta}_{i}\right)+O\left(h^{m+2}\right) .
$$

Now, $\left(z_{\alpha} \dot{\theta}\right)_{i}^{p}=z_{\alpha}\left(\alpha_{i}\right) \dot{\theta}_{i}^{p}+\mathrm{G}_{\mathrm{h}}^{\mathrm{p}} \dot{\theta}_{i}$, where $\mathrm{G}_{\mathrm{h}}^{\mathrm{p}}$ is the commutator of the filtering, that is

$$
\mathrm{G}_{\mathrm{h}}^{\mathrm{p}}\left(\phi_{i}\right) \equiv\left(f\left(\alpha_{i}\right) \phi_{i}\right)^{p}-f\left(\alpha_{i}\right) \phi_{i}^{p}
$$

for smooth functions $f(\cdot)$. Using Lemma 1 , we get

$$
\begin{aligned}
-\frac{\gamma\left(\alpha_{i}\right)}{2 i z_{\alpha}^{2}\left(\alpha_{i}\right)} \mathrm{H}_{\mathrm{h}} \mathrm{D}_{\mathrm{h}} \dot{z}_{i}= & -\frac{\gamma\left(\alpha_{i}\right)}{2 z_{\alpha}\left(\alpha_{i}\right)} \mathrm{H}_{\mathrm{h}} \dot{\theta}_{i}^{p}-\frac{\gamma\left(\alpha_{i}\right)}{2 z_{\alpha}^{2}\left(\alpha_{i}\right)} \mathrm{H}_{\mathrm{h}} \mathrm{G}_{\mathrm{h}}^{\mathrm{p}} \dot{\theta}_{i} \\
& +A_{-2}\left(\dot{\sigma}_{i}, \dot{\theta}_{i}\right)+O\left(h^{m+2}\right) .
\end{aligned}
$$

The term $\mathrm{G}_{\mathrm{h}}^{\mathrm{p}} \dot{\theta}_{i}$ is not smooth enough to be included in $A_{-2}\left(\dot{\sigma}_{i}, \dot{\theta}_{i}\right)$. More precisely, we have the next result whose proof can be found in [15].

\section{Lemma 3.}

$$
\mathrm{G}_{\mathrm{h}}^{\mathrm{p}}\left(\phi_{i}\right)=h A_{0}\left(\phi_{i}\right)
$$

The specific form of the operator $\mathrm{G}_{\mathrm{h}}^{\mathrm{p}}$ will become important in the energy estimates involving the evolution equation for $\dot{\theta}$.

To complete the estimates for $\dot{W}$, we need to take care of its nonlinear part. This is the content of the following lemma adapted from [7]. The details of the proof are given in [15].

Lemma 4. For $t \leq T^{*}$ the nonlinear part of $\dot{W}$ is of the form

$$
\dot{W}_{i}^{N L}=A_{-2}\left(\dot{\sigma}_{i}, \dot{\theta}_{i}, \dot{\tilde{\gamma}}_{i}\right) \text {. }
$$

Now collect all the estimates for $\dot{W}_{i}$. Combining (48), (52), (53), (55), and Lemma 4 we obtain our final estimate for $\dot{W}$.

$$
\begin{aligned}
\dot{W}_{i}= & \frac{\sigma\left(\alpha_{i}\right)}{2 i z_{\alpha}(\alpha)} \mathrm{H}_{\mathrm{h}}\left(\dot{\tilde{\gamma}}_{i}^{p}\right)-\frac{\gamma\left(\alpha_{i}\right)}{2 z_{\alpha}\left(\alpha_{i}\right)} \mathrm{H}_{\mathrm{h}} \dot{\theta}_{i}^{p}+f\left(\alpha_{i}\right) \mathrm{H}_{\mathrm{h}} \mathrm{G}_{\mathrm{h}}^{\mathrm{p}} \dot{\theta}_{i}+E\left(\dot{\theta}_{i}\right) \\
& +A_{-2}\left(\dot{\sigma}_{i}, \dot{\theta}_{i}, \dot{\tilde{\gamma}}_{i}\right)+O\left(h^{m+2}\right),
\end{aligned}
$$

where we have defined

$$
E\left(\dot{\theta}_{i}\right) \equiv f\left(\alpha_{i}\right) \operatorname{Int}_{\mathrm{h}}\left(z_{\alpha} \dot{\theta}\right)_{i}^{p}+g\left(\alpha_{i}\right) \mathrm{H}_{\mathrm{h}} \operatorname{Int}_{\mathrm{h}}\left(z_{\alpha} \dot{\theta}\right)_{i}^{p}
$$

which comes from the last two terms in $(53)$. Hereafter, by $E(\dot{\theta})$ we mean an operator of the form given by the last expression or the real (imaginary) part of it; only the structure of the operator $E$ will be relevant in the final energy estimates.

We can proceed now to estimate the variations of tangential and normal velocities $U^{T}$ and $U^{N}$. We have that

$$
\begin{aligned}
\dot{U}_{i}^{T} & \equiv \operatorname{Re}\left\{e^{i \theta_{i}^{p}} W_{i}-e^{i \theta^{p}\left(\alpha_{i}\right)} W_{h}\left(\alpha_{i}\right)\right\} \\
& =\operatorname{Re}\left\{e^{i \theta^{p}\left(\alpha_{i}\right)} \dot{W}_{i}+W_{h}\left(\alpha_{i}\right)\left(e^{i \theta^{p}}\right)_{i}+\dot{W}_{i}\left(e^{i \theta^{p}}\right)_{i}\right\},
\end{aligned}
$$

where $\left(e^{i \theta^{p}}\right)_{i} \equiv e^{i \theta_{i}^{p}}-e^{i \theta^{p}\left(\alpha_{i}\right)}$. It is clear that (44) remains valid also for $\theta^{p}$. Therefore

$$
\left(e^{i \theta^{p}}\right)_{i}=i e^{i \theta\left(\alpha_{i}\right)} \dot{\theta}_{i}^{p}+A_{-3}\left(\dot{\theta}_{i}\right)
$$


Moreover, using the estimate (56), we can obtain the following bound for $\dot{W}$,

$$
\|\dot{W}\|_{l^{2}} \leq C\left(\|\dot{\sigma}\|_{l^{2}}+\|\dot{\theta}\|_{l^{2}}+\|\dot{\gamma}\|_{l^{2}}\right) .
$$

Thus, $\|\dot{W}\|_{\infty} \leq C h^{3}$, and as a result

$$
\left\|\dot{W}\left(e^{i \theta^{p}}\right)^{\cdot}\right\|_{l_{2}} \leq C\|\dot{W}\|_{\infty}\left\|\left(e^{i \theta^{p}}\right)^{\cdot}\right\|_{l_{2}} \leq C h^{3}\left\|\left(e^{i \theta^{p}}\right)^{\cdot}\right\|_{l_{2}} .
$$

From (58), we conclude that $\dot{W}_{i}\left(e^{i \theta^{p}}\right)_{i}=A_{-3}\left(\dot{\theta}_{i}\right)$, and since $W_{h}=W+O\left(h^{m+2}\right)$, it follows that

$$
\begin{aligned}
\dot{U}_{i}^{T} & =\operatorname{Re}\left\{e^{i \theta^{p}\left(\alpha_{i}\right)} \dot{W}_{i}\right\}-\operatorname{Im}\left\{e^{i \theta\left(\alpha_{i}\right)} W\left(\alpha_{i}\right)\right\} \dot{\theta}_{i}^{p}+A_{-3}\left(\dot{\theta}_{i}\right) \\
& =\left(1+O\left(h^{m+3}\right)\right) \operatorname{Re}\left\{\frac{z_{\alpha}\left(\alpha_{i}\right)}{\sigma\left(\alpha_{i}\right)} \dot{W}_{i}\right\}+U^{N}\left(\alpha_{i}\right) \dot{\theta}_{i}^{p}+A_{-3}\left(\dot{\theta}_{i}\right) .
\end{aligned}
$$

Substitute now our estimate (56) for $\dot{W}$ into this last equation to finally get,

$$
\begin{aligned}
\dot{U}_{i}^{T} & =-\frac{1}{2} \tilde{\gamma}\left(\alpha_{i}\right) \mathrm{H}_{\mathrm{h}} \dot{\theta}_{i}^{p}+U^{N}\left(\alpha_{i}\right) \dot{\theta}_{i}^{p}+f\left(\alpha_{i}\right) \mathrm{H}_{\mathrm{h}} \mathrm{G}_{\mathrm{h}}^{\mathrm{p}} \dot{\theta}_{i}+E\left(\dot{\theta}_{i}\right) \\
& +A_{-2}\left(\dot{\sigma}_{i}, \dot{\theta}_{i}, \dot{\tilde{\gamma}}_{i}\right)+O\left(h^{m+2}\right) .
\end{aligned}
$$

The variation of the normal component of the velocity is estimated similarly. We obtain

$$
\begin{aligned}
\dot{U}_{i}^{N} & =\frac{1}{2} \mathrm{H}_{\mathrm{h}} \dot{\tilde{\gamma}}_{i}^{p}-U^{T}\left(\alpha_{i}\right) \dot{\theta}_{i}^{p}+f\left(\alpha_{i}\right) \mathrm{H}_{\mathrm{h}} \mathrm{G}_{\mathrm{h}}^{\mathrm{p}} \dot{\theta}_{i}+E\left(\dot{\theta}_{i}\right) \\
& +A_{-2}\left(\dot{\sigma}_{i}, \dot{\theta}_{i}, \dot{\tilde{\gamma}}_{i}\right)+O\left(h^{m+2}\right) .
\end{aligned}
$$

Recall that $f(\cdot)$ denotes different smooth functions whose forms we need not specify.

Finally, the variation of the added tangential velocity $U^{A}$ has to be estimated;

$$
\begin{aligned}
\dot{U}_{i}^{A} & =-\dot{U}_{i}^{T}+\operatorname{Int}_{\mathrm{h}}\left(U^{N}(\cdot) \mathrm{D}_{\mathrm{h}} \dot{\theta}\right)_{i}+\operatorname{Int}_{\mathrm{h}}\left(\mathrm{D}_{\mathrm{h}} \theta(\cdot) \dot{U}^{N}\right)_{i} \\
& -\operatorname{Int}_{\mathrm{h}}(R(\cdot))_{i}\left\langle U^{N}(\cdot) \mathrm{D}_{\mathrm{h}} \dot{\theta}\right\rangle_{h}-\operatorname{Int}_{\mathrm{h}}(R(\cdot))_{i}\left\langle\mathrm{D}_{\mathrm{h}} \theta(\cdot) \dot{U}^{N}\right\rangle_{h}+\dot{U}_{i}^{A, N L},
\end{aligned}
$$

where the nonlinear part of the variation is given by

$$
\dot{U}_{i}^{A, N L}=\operatorname{Int}_{\mathrm{h}}\left(\dot{U}^{N} \mathrm{D}_{\mathrm{h}} \dot{\theta}\right)_{i}-\operatorname{Int}_{\mathrm{h}}(R(\cdot))_{i}\left\langle\dot{U}^{N} \mathrm{D}_{\mathrm{h}} \dot{\theta}\right\rangle_{h} .
$$

Note that (60) and our definition of $T^{*}$ imply that $\left\|\dot{U}_{i}^{N}\right\|_{\infty} \leq C h^{3}$. Therefore

$$
\left\|\dot{U}_{i}^{N} \mathrm{D}_{\mathrm{h}} \dot{\theta}\right\|_{l^{2}} \leq\left\|\dot{U}_{i}^{N}\right\|_{\infty} h^{-1}\|\dot{\theta}\|_{l^{2}} \leq C h^{2}\|\dot{\theta}\|_{l^{2}}
$$

from which it follows that $\dot{U}_{i}^{A, N L}=A_{-2}\left(\dot{\theta}_{i}\right)$. Now, from Parseval's identity,

$$
\left\langle U^{N}(\cdot) \mathrm{D}_{\mathrm{h}} \dot{\theta}\right\rangle_{h}=-\left\langle\mathrm{D}_{\mathrm{h}} U^{N}(\cdot) \dot{\theta}\right\rangle_{h}=A_{-m}\left(\dot{\theta}_{i}\right)
$$

and similarly we conclude that

$$
\left\langle\mathrm{D}_{\mathrm{h}} \theta(\cdot) \dot{U}^{N}\right\rangle_{h}=A_{-m}\left(\dot{\sigma}_{i}, \dot{\theta}_{i}, \dot{\tilde{\gamma}}_{i}\right) .
$$

Thus, an estimate for $\dot{U}_{i}^{A}$ can be written in the following form

$$
\dot{U}_{i}^{A}=-\dot{U}_{i}^{T}+B\left(\dot{\sigma}_{i}, \dot{\theta}_{i}, \dot{\tilde{\gamma}}_{i}\right)+A_{-m}\left(\dot{\sigma}_{i}, \dot{\theta}_{i}, \dot{\tilde{\gamma}}_{i}\right),
$$

where

$$
B\left(\dot{\sigma}_{i}, \dot{\theta}_{i}, \dot{\tilde{\gamma}}_{i}\right) \equiv \operatorname{Int}_{\mathrm{h}}\left(U^{N}(\cdot) \mathrm{D}_{\mathrm{h}} \dot{\theta}\right)_{i}+\operatorname{Int}_{\mathrm{h}}\left(\theta_{\alpha}(\cdot) \dot{U}^{N}\right)_{i} .
$$

We would like to "integrate by parts" to obtain the leading order terms of $B$. At the continuous level, integration by parts follows from the product rule $(f g)_{\alpha}=$ $f g_{\alpha}+f_{\alpha} g$. However, due to aliasing, $\mathrm{S}_{\mathrm{h}}(f g)_{i} \neq f_{i} \mathrm{~S}_{\mathrm{h}} g_{i}+g_{i} \mathrm{~S}_{\mathrm{h}} f_{i}$. Of course, we do 
not need this to be satisfied exactly but only that the error be a lower order term. This is the content of the following lemma [7].

Lemma 5. Let $f(\cdot) \in C^{3}$ and $\phi \in l^{2}$, then

$$
\mathrm{D}_{\mathrm{h}}(f(\cdot) \phi)_{i}=f\left(\alpha_{i}\right) \mathrm{D}_{\mathrm{h}} \phi_{i}+f_{\alpha}\left(\alpha_{i}\right) \phi_{i}^{q}+h A_{0}\left(\phi_{i}\right)
$$

where

$$
\hat{\phi}_{k}^{q}=q(k h) \hat{\phi}_{k} ; q(x)=\frac{d}{d x}(x \rho(x)) \text {. }
$$

Hence,

$$
\begin{aligned}
\operatorname{Int}_{\mathrm{h}}\left(U^{N}(\cdot) \mathrm{D}_{\mathrm{h}} \dot{\theta}\right)_{i} & =\operatorname{Int}_{\mathrm{h}}\left(\mathrm{D}_{\mathrm{h}}\left(U^{N}(\cdot) \dot{\theta}\right)\right)_{i}+\operatorname{Int}_{\mathrm{h}}\left(U_{\alpha}^{N}(\cdot) \dot{\theta}^{q}+h A_{0}(\dot{\theta})\right)_{i} \\
& =\left(U^{N}\left(\alpha_{i}\right) \dot{\theta}_{i}\right)^{p}+A_{-1}\left(\dot{\theta}_{i}\right)=U^{N}\left(\alpha_{i}\right) \dot{\theta}_{i}^{p}+A_{-1}\left(\dot{\theta}_{i}\right),
\end{aligned}
$$

where we have used Lemma 3 for the last equality. Now, the estimate (60) for $\dot{U}^{N}$ in the other term of $B$, gives us

$$
B\left(\dot{\sigma}_{i}, \dot{\theta}_{i}, \dot{\tilde{\gamma}}_{i}\right)=U^{N}\left(\alpha_{i}\right) \dot{\theta}_{i}^{p}+A_{-1}\left(\dot{\sigma}_{i}, \dot{\theta}_{i}, \dot{\tilde{\gamma}}_{i}\right)
$$

Therefore,

$$
\dot{U}_{i}^{A}=-\dot{U}_{i}^{T}+U^{N}\left(\alpha_{i}\right) \dot{\theta}_{i}^{p}+A_{-1}\left(\dot{\sigma}_{i}, \dot{\theta}_{i}, \dot{\tilde{\gamma}}_{i}\right)
$$

5.2. Evolution Equations for the Errors. We use now our estimates for the velocity variations and the Consistency Lemma to find evolution equations for the errors. The most technical part is the equation for $\dot{\tilde{\gamma}}$. In addition to estimating numerous linear and nonlinear terms, we also have to prove the solvability of a discrete integral equation for $d \dot{\gamma} / d t$. Of course, this would not be necessary if the Atwood number $A$ were equal to zero.

It is easy to obtain an evolution equation for $\dot{\sigma}$. Using the Consistency Lemma we have,

$$
\begin{aligned}
\frac{d \dot{\sigma}_{i}}{d t} & =-R\left(\alpha_{i}\right)\left\langle\mathrm{S}_{\mathrm{h}} \theta U^{N}-\mathrm{S}_{\mathrm{h}} \theta(\cdot) U^{N}(\cdot)\right\rangle_{h}+O\left(h^{m+2}\right) \\
& =R\left(\alpha_{i}\right)\left\langle\mathrm{S}_{\mathrm{h}} U^{N}(\cdot) \dot{\theta}-\mathrm{S}_{\mathrm{h}} \theta(\cdot) \dot{U}^{N}-\mathrm{S}_{\mathrm{h}} \dot{\theta} \dot{U}^{N}\right\rangle_{h}+O\left(h^{m+2}\right) .
\end{aligned}
$$

The nonlinear term can be bound just as in (61). It follows that

$$
\frac{d \dot{\sigma}_{i}}{d t}=A_{-2}\left(\dot{\sigma}_{i}, \dot{\theta}_{i}, \dot{\tilde{\gamma}}_{i}\right)+O\left(h^{m+2}\right) \text {. }
$$

As we mentioned before, equation (37) for $\dot{\theta}$ can be written in terms of $\dot{\sigma}, \dot{\theta}$ and the velocity variations $\dot{U}^{N}, \dot{U}^{T}$ and $\dot{U}^{A}$. By direct computation, and estimating the nonlinear terms just as we did for $\dot{U}^{N}$, we get (see [15] for details)

$$
\begin{aligned}
\frac{d \dot{\theta}_{i}}{d t} & =\frac{1}{\sigma\left(\alpha_{i}\right)} \mathrm{S}_{\mathrm{h}} \dot{U}_{i}^{N}+\frac{U^{T}\left(\alpha_{i}\right)+U^{A}\left(\alpha_{i}\right)}{\sigma\left(\alpha_{i}\right)} \mathrm{D}_{\mathrm{h}} \dot{\theta}_{i}+\frac{\theta_{\alpha}\left(\alpha_{i}\right) U^{N}\left(\alpha_{i}\right)}{\sigma\left(\alpha_{i}\right)} \dot{\theta}_{i}^{p} \\
& +A_{-2}\left(\dot{\theta}_{i}, \dot{\sigma}_{i}, \dot{\tilde{\gamma}}_{i}\right)+O\left(h^{m+1}\right)
\end{aligned}
$$

where we have also used (63). We estimate $\mathrm{S}_{\mathrm{h}} \dot{U}_{i}^{N}$ from (60);

$$
\begin{aligned}
\mathrm{S}_{\mathrm{h}} \dot{U}_{i}^{N} & =\frac{1}{2} \Lambda_{\mathrm{h}} \dot{\tilde{\gamma}}_{i}-U^{T}\left(\alpha_{i}\right) \mathrm{D}_{\mathrm{h}} \dot{\theta}_{i}-U_{\alpha}^{T}\left(\alpha_{i}\right) \dot{\theta}_{i}^{q}+\mathrm{S}_{\mathrm{h}} E\left(\dot{\theta}_{i}\right) \\
& +\mathrm{S}_{\mathrm{h}} \mathrm{G}_{\mathrm{h}}^{\mathrm{p}} \dot{\theta}_{i}+\mathrm{S}_{\mathrm{h}} f\left(\alpha_{i}\right) \mathrm{H}_{\mathrm{h}} \mathrm{G}_{\mathrm{h}}^{\mathrm{p}} \dot{\theta}_{i}+A_{-1}\left(\dot{\theta}_{i}, \dot{\sigma}_{i}, \dot{\tilde{\gamma}}_{i}\right)+O\left(h^{m+1}\right),
\end{aligned}
$$


where we have combined (54) and Lemma 5 in applying the operator $\mathrm{S}_{\mathrm{h}}$ to $-U^{T} \dot{\theta}^{p}$, and have defined $\Lambda_{\mathrm{h}} \equiv \mathrm{H}_{\mathrm{h}} \mathrm{D}_{\mathrm{h}}$. Here is where the use of $\tilde{\gamma}$ makes a difference; the operator $\Lambda_{\mathrm{h}}$ is acting on $\dot{\tilde{\gamma}}$ only, and the other terms in $\dot{\tilde{\gamma}}$ are $A_{-1}$, just what we need for the balance in the energy estimates below. Finally, substituting (66) into (65) we get

$$
\begin{aligned}
\frac{d \dot{\theta}_{i}}{d t} & =\frac{1}{2 \sigma\left(\alpha_{i}\right)} \Lambda_{\mathrm{h}} \dot{\tilde{\gamma}}_{i}+\frac{U^{A}\left(\alpha_{i}\right)}{\sigma\left(\alpha_{i}\right)} \mathrm{D}_{\mathrm{h}} \dot{\theta}_{i}+f\left(\alpha_{i}\right) \dot{\theta}_{i}^{p}+g\left(\alpha_{i}\right) \dot{\theta}_{i}^{q} \\
& +\frac{1}{\sigma\left(\alpha_{i}\right)} \mathrm{S}_{\mathrm{h}} E\left(\dot{\theta}_{i}\right)+\frac{1}{\sigma\left(\alpha_{i}\right)} \mathrm{S}_{\mathrm{h}} \mathrm{G}_{\mathrm{h}}^{\mathrm{p}} \dot{\theta}_{i}+\frac{1}{\sigma\left(\alpha_{i}\right)} \mathrm{S}_{\mathrm{h}} f\left(\alpha_{i}\right) \mathrm{H}_{\mathrm{h}} \mathrm{G}_{\mathrm{h}}^{\mathrm{p}} \dot{\theta}_{i} \\
& +A_{-1}\left(\dot{\theta}_{i}, \dot{\sigma}_{i}, \dot{\tilde{\gamma}}_{i}\right)+O\left(h^{m+1}\right) .
\end{aligned}
$$

Obtaining an evolution equation for $\dot{\tilde{\gamma}}$ requires estimating many terms. However, only a few of these terms contribute to the leading order behavior. More precisely, we will show that

$$
\begin{aligned}
\frac{d \dot{\tilde{\gamma}}_{i}}{d t}= & \frac{S}{\sigma\left(\alpha_{i}\right)} \mathrm{D}_{\mathrm{h}} \frac{1}{\sigma\left(\alpha_{i}\right)} \mathrm{D}_{\mathrm{h}} \dot{\theta}_{i}+\frac{\tilde{\gamma}^{2}\left(\alpha_{i}\right)}{2 \sigma\left(\alpha_{i}\right)} \Lambda_{\mathrm{h}} \dot{\theta}_{i}^{p}+f\left(\alpha_{i}\right) \mathrm{D}_{\mathrm{h}} \dot{\tilde{\gamma}}_{i} \\
& +A_{0}\left(\dot{\sigma}_{i}, \dot{\theta}_{i}, \dot{\tilde{\gamma}}_{i}\right)+O\left(h^{m}\right) .
\end{aligned}
$$

First, we obtain an evolution equation for $\dot{\gamma}$. In view of the Consistency Lemma and (27),

$$
\frac{d \dot{\gamma}_{i}}{d t}=\dot{Q}_{i}+\dot{F}_{i}+O\left(h^{m}\right)
$$

where

$$
\begin{aligned}
\dot{Q}_{i} & \equiv S \mathrm{D}_{\mathrm{h}}\left(\frac{1}{\sigma_{i}} \mathrm{D}_{\mathrm{h}} \theta_{i}\right)-S \mathrm{D}_{\mathrm{h}}\left(\frac{1}{\sigma\left(\alpha_{i}\right)} \mathrm{D}_{\mathrm{h}} \theta\left(\alpha_{i}\right)\right)+\mathrm{D}_{\mathrm{h}}\left(U_{i}^{A} \tilde{\gamma}_{i}\right)-\mathrm{D}_{\mathrm{h}}\left(U_{h}^{A}\left(\alpha_{i}\right) \tilde{\gamma}\left(\alpha_{i}\right)\right) \\
& -2 A\left[\frac{1}{8} \mathrm{D}_{\mathrm{h}} \tilde{\gamma}_{i}^{2}+g \sigma_{i} \sin \theta_{i}-U^{A} \operatorname{Re}\left\{e^{i \theta_{i}} \mathrm{~S}_{\mathrm{h}} W_{i}\right\}\right] \\
& +2 A\left[\frac{1}{8} \mathrm{D}_{\mathrm{h}} \tilde{\gamma}^{2}\left(\alpha_{i}\right)+g \sigma\left(\alpha_{i}\right) \sin \theta\left(\alpha_{i}\right)-U_{h}^{A}\left(\alpha_{i}\right) \operatorname{Re}\left\{e^{i \theta\left(\alpha_{i}\right)} \mathrm{S}_{\mathrm{h}} W_{h}\left(\alpha_{i}\right)\right\}\right],
\end{aligned}
$$

and

$$
\dot{F}_{i} \equiv F_{i}-F_{h}\left(\alpha_{i}\right),
$$

where $F_{i}$ is given by $(28)$ and $F_{h}\left(\alpha_{i}\right)$ is the corresponding expression obtained by substituting the exact solution into $(28)$. While $\dot{Q}_{i}$ contains many terms, they can be estimated with the same procedure as in the evolution equation for $\dot{\theta}$. The details of the estimates are given in Chapter 3 of [15]. We find that

$$
\begin{aligned}
\dot{Q}_{i} & =S \mathrm{D}_{\mathrm{h}}\left(\frac{1}{\sigma_{i}} \mathrm{D}_{\mathrm{h}} \dot{\theta}_{i}\right)+\frac{1}{2} \tilde{\gamma}^{2}\left(\alpha_{i}\right) \Lambda_{\mathrm{h}} \dot{\theta}_{i}^{p}-A \tilde{\gamma}\left(\alpha_{i}\right) U^{A}\left(\alpha_{i}\right) \Lambda_{\mathrm{h}} \dot{\theta}_{i} \\
& +\left[U^{A}\left(\alpha_{i}\right)-A \tilde{\gamma}\left(\alpha_{i}\right)\right] \mathrm{D}_{\mathrm{h}} \dot{\tilde{\gamma}}_{i}+A_{0}\left(\dot{\sigma}_{i}, \dot{\theta}_{i}, \dot{\tilde{\gamma}}_{i}\right) .
\end{aligned}
$$

Recall that $\Lambda_{\mathrm{h}}=\mathrm{H}_{\mathrm{h}} \mathrm{D}_{\mathrm{h}}$. The estimate of $\dot{F}_{i}$ is different and requires one important technical result: the solvability of a discrete integral equation. We start first by splitting $\dot{F}_{i}$ into linear and nonlinear parts in the variations,

$$
\dot{F}_{i}=\dot{F}_{i}^{L}+\dot{F}_{i}^{N L}
$$


where $\dot{F}_{i}^{L}$ contains linear terms in the variations. It is not difficult, although very lengthy to show [15], that $\dot{F}_{i}^{N L}=A_{0}\left(\dot{\sigma}_{i}, \dot{\theta}_{i}, \dot{\gamma}_{i}\right)$, and

$$
\begin{aligned}
\dot{F}_{i}^{L} & =-2 A R e\left\{\frac{z_{\alpha}\left(\alpha_{i}\right)}{2 \pi i} \sum_{(j-i) \text { odd }} \frac{\frac{d \dot{\gamma}_{j}}{d t}}{z_{h}^{p}\left(\alpha_{i}\right)-z_{h}^{p}\left(\alpha_{j}\right)} 2 h\right\} \\
& +2 A R e\left\{\frac{z_{\alpha}\left(\alpha_{i}\right)}{2 \pi i} \sum_{(j-i) \text { odd }} \gamma^{p}\left(\alpha_{j}\right) \frac{\frac{d \dot{z}_{i}}{d t}-\frac{d \dot{z}_{j}}{d t}}{\left(z_{h}^{p}\left(\alpha_{i}\right)-z_{h}^{p}\left(\alpha_{j}\right)\right)^{2}} 2 h\right\}+A_{0}\left(\dot{\sigma}_{i}, \dot{\theta}_{i}, \dot{\tilde{\gamma}}_{i}\right)
\end{aligned}
$$

The leading order part of $\dot{F}_{i}$ is contained in the second term of the right hand side of (70). This term can be estimated just as we did to get (53) for one of the terms in $\dot{W}_{i}$. Thus,

$$
\begin{aligned}
\dot{F}_{i}^{(2)} & \equiv 2 A \operatorname{Re}\left\{\frac{z_{\alpha}\left(\alpha_{i}\right)}{2 \pi i} \sum_{(j-i) \text { odd }} \gamma^{p}\left(\alpha_{j}\right) \frac{\frac{d \dot{z}_{i}}{d t}-\frac{d \dot{z}_{j}}{d t}}{\left(z_{h}^{p}\left(\alpha_{i}\right)-z_{h}^{p}\left(\alpha_{j}\right)\right)^{2}} 2 h\right\} \\
& =2 A R e\left\{\frac{\frac{d \gamma}{d t}\left(\alpha_{i}\right)}{2 \pi i z_{\alpha}\left(\alpha_{i}\right)} \sum_{(j-i) \text { odd }} \frac{\frac{d \dot{z}_{i}}{d t}-\frac{d \dot{z}_{j}}{d t}}{\left(\alpha_{i}-\alpha_{j}\right)^{2}} 2 h\right\}+A_{0}\left(\frac{d \dot{z}_{i}}{d t}\right) .
\end{aligned}
$$

We now need an estimate for $\frac{d \dot{z}_{i}}{d t}$. From (29), (64), and (67) it follows that

$$
\begin{aligned}
\frac{d \dot{z}_{i}}{d t} & =\operatorname{Int}_{\mathrm{h}}\left[i z_{\alpha}(\cdot) \frac{d \dot{\theta}}{d t}+e^{i \theta(\cdot)} \frac{d \dot{\sigma}}{d t}+A_{0}(\dot{\sigma}, \dot{\theta})\right]_{i}+O\left(h^{m+1}\right) \\
& =\operatorname{Int}_{\mathrm{h}}\left[i \frac{z_{\alpha}(\cdot)}{2 \sigma(\cdot)} \Lambda_{\mathrm{h}} \dot{\tilde{\gamma}}+i \frac{U^{A}(\cdot) z_{\alpha}(\cdot)}{\sigma(\cdot)} \mathrm{D}_{\mathrm{h}} \dot{\theta}+A_{0}(\dot{\sigma}, \dot{\theta})\right]_{i}+O\left(h^{m+1}\right)
\end{aligned}
$$

and "integrating by parts" on $\dot{\tilde{\gamma}}$ and $\dot{\theta}$ we get

$$
\frac{d \dot{z}_{i}}{d t}=i\left(\frac{z_{\alpha}}{2 \sigma} \mathrm{H}_{\mathrm{h}} \dot{\tilde{\gamma}}_{i}\right)_{i}^{p}+i\left(U^{A} \frac{z_{\alpha}}{\sigma} \dot{\theta}_{i}\right)_{i}^{p}+\operatorname{Int}_{\mathrm{h}}\left[A_{0}(\dot{\sigma}, \dot{\theta}, \dot{\tilde{\gamma}})\right]_{i}+O\left(h^{m+1}\right) .
$$

Substituting (72) into (71) and using Lemma 2 we find that

$$
\dot{F}_{i}^{(2)}=-\frac{1}{2} A \tilde{\gamma}\left(\alpha_{i}\right) \mathrm{D}_{\mathrm{h}} \dot{\tilde{\gamma}}_{i}+A \tilde{\gamma}\left(\alpha_{i}\right) U^{A}\left(\alpha_{i}\right) \Lambda_{\mathrm{h}} \dot{\theta}_{i}+A_{0}\left(\dot{\sigma}_{i}, \dot{\theta}_{i}, \dot{\tilde{\gamma}}_{i}\right)+O\left(h^{m}\right) .
$$

Therefore, we can conclude that

$$
\begin{aligned}
\dot{F}_{i} & =-\mathrm{K}_{\mathrm{h}} \frac{d \dot{\gamma}_{i}}{d t}-\frac{1}{2} A \tilde{\gamma}\left(\alpha_{i}\right) \mathrm{D}_{\mathrm{h}} \dot{\tilde{\gamma}}_{i}+A \tilde{\gamma}\left(\alpha_{i}\right) U^{A}\left(\alpha_{i}\right) \Lambda_{\mathrm{h}} \dot{\theta}_{i} \\
& +A_{0}\left(\dot{\sigma}_{i}, \dot{\theta}_{i}, \dot{\tilde{\gamma}}_{i}\right)+O\left(h^{m}\right)
\end{aligned}
$$

where

$$
\mathrm{K}_{\mathrm{h}} \frac{d \dot{\gamma}_{i}}{d t} \equiv 2 A R e\left\{\frac{z_{\alpha}\left(\alpha_{i}\right)}{2 \pi i} \sum_{(j-i) \text { odd }} \frac{\frac{d \dot{\gamma}_{j}}{d t}}{z_{h}^{p}\left(\alpha_{i}\right)-z_{h}^{p}\left(\alpha_{j}\right)} 2 h\right\} .
$$

Substitute now the estimates (69) and (73) for $\dot{Q}_{i}$ and $\dot{F}_{i}$ into (68) to obtain

$$
\left(\mathrm{I}+\mathrm{K}_{\mathrm{h}}\right) \frac{d \dot{\gamma}_{i}}{d t}=P\left(\dot{\theta}_{i}, \dot{\tilde{\gamma}}_{i}\right)+A_{0}\left(\dot{\sigma}_{i}, \dot{\theta}_{i}, \dot{\gamma}_{i}\right)+O\left(h^{m}\right),
$$

where

$$
P\left(\dot{\theta}_{i}, \dot{\tilde{\gamma}}_{i}\right)=S \mathrm{D}_{\mathrm{h}}\left(\frac{1}{\sigma\left(\alpha_{i}\right)} \mathrm{D}_{\mathrm{h}} \dot{\theta}_{i}\right)+\frac{1}{2} \tilde{\gamma}^{2}\left(\alpha_{i}\right) \Lambda_{\mathrm{h}} \dot{\theta}_{i}^{p}+f\left(\alpha_{i}\right) \mathrm{D}_{\mathrm{h}} \dot{\tilde{\gamma}}_{i} .
$$


Note that (75) is a discrete version of a Fredholm integral equation of second kind. The following result shows that the operator $\mathrm{I}+\mathrm{K}_{\mathrm{h}}$ has a bounded inverse in $l^{2}[7$, $15]$.

Lemma 6. Assume that $z(\cdot ; t) \in C^{3}$ and $z_{\alpha} \neq 0$. Then there exist constants $h_{0}>0$ and $C>0$ such that for all $h$ satisfying $0<h \leq h_{0}$,

$$
\left\|\left(\mathrm{I}+\mathrm{K}_{\mathrm{h}}\right)^{-1}\right\|_{l^{2}} \leq C .
$$

We adapt now the argument of BHL [7], to show that $\mathrm{K}_{\mathrm{h}} \frac{d \dot{\gamma}_{i}}{d t}$ is actually a lower order term. Indeed,

$$
\begin{aligned}
\mathrm{K}_{\mathrm{h}} \frac{d \dot{\gamma}_{i}}{d t} & =\mathrm{K}_{\mathrm{h}}\left(\mathrm{I}+\mathrm{K}_{\mathrm{h}}\right)^{-1}\left[P\left(\dot{\theta}_{i}, \dot{\tilde{\gamma}}_{i}\right)+A_{0}\left(\dot{\sigma}_{i}, \dot{\theta}_{i}, \dot{\gamma}_{i}\right)+O\left(h^{m}\right)\right] \\
& =\left(\mathrm{I}+\mathrm{K}_{\mathrm{h}}\right)^{-1} \mathrm{~K}_{\mathrm{h}} P\left(\dot{\theta}_{i}, \dot{\tilde{\gamma}}_{i}\right)+A_{0}\left(\dot{\sigma}_{i}, \dot{\theta}_{i}, \dot{\gamma}_{i}\right)+O\left(h^{m}\right),
\end{aligned}
$$

where we have used commutability of the operators, that is

$$
\mathrm{K}_{\mathrm{h}}\left(\mathrm{I}+\mathrm{K}_{\mathrm{h}}\right)^{-1}=\left(\mathrm{I}+\mathrm{K}_{\mathrm{h}}\right)^{-1} \mathrm{~K}_{\mathrm{h}} .
$$

and the fact that $\mathrm{K}_{\mathrm{h}}$ and $\left(\mathrm{I}+\mathrm{K}_{\mathrm{h}}\right)^{-1}$ are bounded in $l^{2}$. Moreover, note that

$$
\begin{aligned}
\mathrm{K}_{\mathrm{h}} \dot{\phi}_{i} & =\operatorname{Re}\left[\frac{1}{2 \pi i} \sum_{(j-i) \text { odd }} \frac{\dot{\phi}_{j}}{\alpha_{i}-\alpha_{j}} 2 h\right]+\mathrm{R}_{\mathrm{h}}\left(\dot{\phi}_{i}\right)+A_{-m}\left(\dot{\phi}_{i}\right) \\
& =\mathrm{R}_{\mathrm{h}}\left(\dot{\phi}_{i}\right)+A_{-m}\left(\dot{\phi}_{i}\right)
\end{aligned}
$$

where $R_{h}$ is a discrete 'smoothing' operator of the type defined in (50). Here is a place where the right combination of filtering is crucial. By Lemma 1 we know that if Fourier smoothing is applied to eliminate aliasing errors, then $\mathrm{R}_{\mathrm{h}}=A_{-2}$. Therefore,

$$
\begin{aligned}
\mathrm{R}_{\mathrm{h}}\left(\mathrm{D}_{\mathrm{h}} \frac{1}{\sigma\left(\alpha_{i}\right)} \mathrm{D}_{\mathrm{h}} \dot{\theta}_{i}\right) & =A_{-2}\left(\mathrm{~S}_{\mathrm{h}} \frac{1}{\sigma\left(\alpha_{i}\right)} \mathrm{D}_{\mathrm{h}} \dot{\theta}_{i}\right)=A_{0}\left(\dot{\theta}_{i}\right), \\
\mathrm{R}_{\mathrm{h}}\left(\Lambda_{\mathrm{h}} \dot{\theta}_{i}^{p}\right) & =A_{-2}\left(\Lambda_{\mathrm{h}} \dot{\theta}_{i}\right)=A_{0}\left(\dot{\theta}_{i}\right), \\
\mathrm{R}_{\mathrm{h}}\left(\mathrm{D}_{\mathrm{h}} \dot{\tilde{\gamma}}_{i}\right) & =A_{-1}\left(\dot{\tilde{\gamma}}_{i}\right),
\end{aligned}
$$

which shows that

$$
\mathrm{K}_{\mathrm{h}} \frac{d \dot{\gamma}_{i}}{d t}=A_{0}\left(\dot{\sigma}_{i}, \dot{\theta}_{i}, \dot{\tilde{\gamma}}_{i}\right)
$$

Therefore our previous estimate (75) becomes

$$
\begin{aligned}
\frac{d \dot{\gamma}_{i}}{d t} & =S \mathrm{D}_{\mathrm{h}} \frac{1}{\sigma\left(\alpha_{i}\right)} \mathrm{D}_{\mathrm{h}} \dot{\theta}_{i}+\frac{1}{2} \tilde{\gamma}^{2}\left(\alpha_{i}\right) \Lambda_{\mathrm{h}} \dot{\theta}_{i}^{p}+f\left(\alpha_{i}\right) \mathrm{D}_{\mathrm{h}} \dot{\tilde{\gamma}}_{i} \\
& +A_{0}\left(\dot{\sigma}_{i}, \dot{\theta}_{i}, \dot{\tilde{\gamma}}_{i}\right)+O\left(h^{m}\right) .
\end{aligned}
$$

However, we need an evolution equation for $\dot{\tilde{\gamma}}_{i}$. Recall that $\tilde{\gamma}=\gamma / \sigma$, thus

$$
\begin{aligned}
\frac{d \dot{\tilde{\gamma}}_{i}}{d t} & =\frac{1}{\sigma\left(\alpha_{i}\right)} \frac{d \dot{\gamma}_{i}}{d t}+\left(\frac{1}{\sigma}\right)_{i} \frac{d \gamma_{i}}{d t}-\left(\frac{\gamma}{\sigma^{2}} \frac{d \sigma}{d t}\right)_{i} \\
& =\frac{1}{\sigma\left(\alpha_{i}\right)} \frac{d \dot{\gamma}_{i}}{d t}+A_{0}\left(\dot{\sigma}_{i}, \dot{\theta}_{i}, \dot{\tilde{\gamma}}_{i}\right)+O\left(h^{m}\right)
\end{aligned}
$$


where we have used (76). Thus dividing equation (76) by $\sigma\left(\alpha_{i}\right)$ we arrive at

$$
\begin{aligned}
\frac{d \dot{\tilde{\gamma}}_{i}}{d t} & =\frac{S}{\sigma\left(\alpha_{i}\right)} \mathrm{D}_{\mathrm{h}} \frac{1}{\sigma\left(\alpha_{i}\right)} \mathrm{D}_{\mathrm{h}} \dot{\theta}_{i}+\frac{\tilde{\gamma}^{2}\left(\alpha_{i}\right)}{2 \sigma\left(\alpha_{i}\right)} \Lambda_{\mathrm{h}} \dot{\theta}_{i}^{p}+f\left(\alpha_{i}\right) \mathrm{D}_{\mathrm{h}} \dot{\tilde{\gamma}}_{i} \\
& +A_{0}\left(\dot{\sigma}_{i}, \dot{\theta}_{i}, \dot{\tilde{\gamma}}_{i}\right)+O\left(h^{m}\right) .
\end{aligned}
$$

This completes our system of evolution equations for the errors. We have shown that, for $t \leq T^{*}$,

$$
\begin{aligned}
\frac{d \dot{\sigma}_{i}}{d t} & =A_{-2}\left(\dot{\sigma}_{i}, \dot{\theta}_{i}, \dot{\gamma}_{i}\right)+O\left(h^{m+2}\right) \\
\frac{d \dot{\theta}_{i}}{d t} & =\frac{1}{2 \sigma\left(\alpha_{i}\right)} \Lambda_{\mathrm{h}} \dot{\tilde{\gamma}}_{i}+f\left(\alpha_{i}\right) \mathrm{D}_{\mathrm{h}} \dot{\theta}_{i}+\Theta\left(\dot{\theta}_{i}\right)+A_{-1}\left(\dot{\theta}_{i}, \dot{\sigma}_{i}, \dot{\tilde{\gamma}}_{i}\right)+O\left(h^{m+1}\right) \\
\frac{d \dot{\tilde{\gamma}_{i}}}{d t} & =-\frac{S}{\sigma\left(\alpha_{i}\right)} \Lambda_{\mathrm{h}} \frac{1}{\sigma\left(\alpha_{i}\right)}\left(\mathrm{H}_{\mathrm{h}} \mathrm{S}_{\mathrm{h}}-\frac{\gamma^{2}\left(\alpha_{i}\right)}{2 S \sigma\left(\alpha_{i}\right)}\right) \dot{\theta}_{i}^{p}+f\left(\alpha_{i}\right) \mathrm{D}_{\mathrm{h}} \dot{\tilde{\gamma}}_{i} \\
& +A_{0}\left(\dot{\sigma}_{i}, \dot{\theta}_{i}, \dot{\tilde{\gamma}}_{i}\right)+O\left(h^{m}\right),
\end{aligned}
$$

where $\Lambda_{\mathrm{h}}=\mathrm{H}_{\mathrm{h}} \mathrm{D}_{\mathrm{h}}$, and $\Theta\left(\dot{\theta}_{i}\right)$ is of the form

$$
\begin{aligned}
\Theta\left(\dot{\theta}_{i}\right) & =c_{1}\left(\alpha_{i}\right) \dot{\theta}_{i}^{p}+c_{2}\left(\alpha_{i}\right) \dot{\theta}_{i}^{q}+c_{3}\left(\alpha_{i}\right) \mathrm{S}_{\mathrm{h}} \mathrm{G}_{\mathrm{h}}^{\mathrm{p}}\left(\dot{\theta}_{i}\right) \\
& +c_{4}\left(\alpha_{i}\right) \mathrm{S}_{\mathrm{h}} f\left(\alpha_{i}\right) \mathrm{H}_{\mathrm{h}} \mathrm{G}_{\mathrm{h}}^{\mathrm{p}}\left(\dot{\theta}_{i}\right)+c_{5}\left(\alpha_{i}\right) \mathrm{S}_{\mathrm{h}} E\left(\dot{\theta}_{i}\right)
\end{aligned}
$$

and all the $c_{i}$ 's are smooth functions. Recall that $E\left(\dot{\theta}_{i}\right)$, given by (57) comes from the transformation linking $z$ to $\sigma$ and $\theta$ and that $\mathrm{G}_{\mathrm{h}}^{\mathrm{p}}(54)$ is the commutator of the filtering. Note also that in (79), we have combined the two leading order terms in $\dot{\theta}$ into a single term that makes more transparent the stabilizing effect of surface tension.

5.3. Energy Estimates. To illustrate the idea, let us consider only the leading order terms in the evolution equations for $\dot{\theta}_{i}$ and $\dot{\tilde{\gamma}}_{i}$.

$$
\begin{aligned}
\frac{d \dot{\theta}_{i}}{d t} & =\frac{1}{2 \sigma\left(\alpha_{i}\right)} \Lambda_{\mathrm{h}} \dot{\tilde{\gamma}}_{i}+\cdots, \\
\frac{d \dot{\tilde{\gamma}}_{i}}{d t} & =-\frac{S}{\sigma\left(\alpha_{i}\right)} \Lambda_{\mathrm{h}} \frac{1}{\sigma\left(\alpha_{i}\right)}\left(\mathrm{H}_{\mathrm{h}} \mathrm{S}_{\mathrm{h}}-a\left(\alpha_{i}\right)\right) \dot{\theta}_{i}^{p}+\cdots,
\end{aligned}
$$

where $a\left(\alpha_{i}\right)=\gamma^{2}\left(\alpha_{i}\right) / 2 S \sigma\left(\alpha_{i}\right)$. Let us denote the usual discrete inner product as follows

$$
(\phi, \psi)_{h} \equiv h \sum_{j=-N / 2+1}^{N / 2} \phi_{j} \psi_{j} .
$$

Suppose now that we take the inner product of the equation for $\dot{\tilde{\gamma}}_{i}$ and $(\sigma / 2 S) \dot{\tilde{\gamma}}_{i}$. We obtain

$$
\left(\sigma \dot{\tilde{\gamma}}, \frac{d \dot{\tilde{\gamma}}}{d t}\right)_{h}=-\frac{1}{2}\left(\Lambda_{\mathrm{h}} \dot{\tilde{\gamma}}, \frac{1}{\sigma}\left(\mathrm{H}_{\mathrm{h}} \mathrm{S}_{\mathrm{h}}-a\right) \dot{\theta}^{p}\right)_{h}+\cdots
$$

where we have used that $\Lambda_{h}=H_{h} D_{h}$ is a symmetric operator. On the other hand, if we take the inner product of the equation for $\dot{\theta}$ and $\left(\mathrm{H}_{\mathrm{h}} \mathrm{S}_{\mathrm{h}}-a\right) \dot{\theta}^{p}$, we get

$$
\left(\left(\mathrm{H}_{\mathrm{h}} \mathrm{S}_{\mathrm{h}}-a\right) \dot{\theta}^{p}, \frac{d \dot{\theta}}{d t}\right)_{h}=\frac{1}{2}\left(\frac{1}{\sigma} \Lambda_{\mathrm{h}} \dot{\tilde{\gamma}},\left(\mathrm{H}_{\mathrm{h}} \mathrm{S}_{\mathrm{h}}-a\right) \dot{\theta}^{p}\right)_{h}+\cdots
$$


Thus, the leading order terms of these two last equations would balance each other; just as we need for stability. However, $\mathrm{H}_{\mathrm{h}} \mathrm{S}_{\mathrm{h}}-a(\alpha)$ is not a positive operator but instead, its action depends on whether the modes are greater or less than $\|a\|_{\infty}$. In other words, surface tension divides the spectrum in two bands, with its effect becoming dominant for those modes greater than $\|a\|_{\infty}$. Therefore, a careful distinction of the two bands of modes must be made in the energy estimates. To this end, we follow a technique developed by BHL to study the growth rates for the linear motion of a two-fluid interface at the continuous level [8]. We adapt this technique to the discrete case corresponding to our numerical method.

Let $P_{M}$ be the following projection operator

$$
\widehat{\left(P_{M} u\right)_{k}}= \begin{cases}\hat{u}_{k} & \text { if }|k|>M, \\ 0 & \text { if }|k| \leq M,\end{cases}
$$

where $M=2\left(\|a\|_{\infty}+1\right)$. Note that the definition of the projection operator implies that $\left\|P_{M} u\right\|_{l^{2}} \leq\|u\|_{l^{2}}$. With this in mind, let us take the inner product of the whole evolution equation for $\dot{\tilde{\gamma}}(79)$ with $(\sigma / 2 S) \dot{\tilde{\gamma}}$. We get

$$
\begin{aligned}
\frac{1}{2 S}\left(\sigma \dot{\tilde{\gamma}}, \frac{d \dot{\tilde{\gamma}}}{d t}\right)_{h} & =-\frac{1}{2}\left(\Lambda_{\mathrm{h}} \dot{\tilde{\gamma}}, \frac{1}{\sigma}\left(\mathrm{H}_{\mathrm{h}} \mathrm{S}_{\mathrm{h}}-a\right) \dot{\theta}^{p}\right)_{h}+\left(f \dot{\tilde{\gamma}}, \mathrm{D}_{\mathrm{h}} \dot{\tilde{\gamma}}\right)_{h} \\
& +\frac{1}{2 S}\left(\sigma \dot{\tilde{\gamma}}, A_{0}(\dot{\sigma}, \dot{\theta}, \dot{\tilde{\gamma}})+O\left(h^{m}\right)\right)_{h} .
\end{aligned}
$$

Clearly for the last term in the right hand side, we have that

$$
\left|\frac{1}{2 S}\left(\sigma \dot{\tilde{\gamma}}, A_{0}(\dot{\sigma}, \dot{\theta}, \dot{\tilde{\gamma}})+O\left(h^{m}\right)\right)_{h}\right| \leq C\|\dot{\tilde{\gamma}}\|_{l^{2}}\left(\|\dot{\tilde{\gamma}}\|_{l^{2}}+\|\dot{\sigma}\|_{l^{2}}+\|\dot{\theta}\|_{l^{2}}+O\left(h^{m}\right)\right) .
$$

On the other hand,

$$
\left(f \dot{\tilde{\gamma}}, \mathrm{D}_{\mathrm{h}} \dot{\tilde{\gamma}}\right)_{h}=-\left(\mathrm{D}_{\mathrm{h}}(f \dot{\tilde{\gamma}}), \dot{\tilde{\gamma}}\right)_{h}=-\left(f \mathrm{D}_{\mathrm{h}} \dot{\tilde{\gamma}}, \dot{\tilde{\gamma}}\right)_{h}-\left(f_{\alpha} \dot{\tilde{\gamma}}^{q}+h A_{0}(\dot{\tilde{\gamma}}), \dot{\tilde{\gamma}}\right)_{h},
$$

and thus

$$
\left|\left(f \dot{\tilde{\gamma}}, \mathrm{D}_{\mathrm{h}} \dot{\tilde{\gamma}}\right)_{h}\right|=\frac{1}{2}\left|\left(f_{\alpha} \dot{\tilde{\gamma}}^{q}+h A_{0}(\dot{\tilde{\gamma}}), \dot{\tilde{\gamma}}\right)_{h}\right| \leq C\|\dot{\tilde{\gamma}}\|_{l^{2}}^{2}
$$

Now, we separate the modes for the leading order term;

$$
\begin{aligned}
-\frac{1}{2}\left(\frac{1}{\sigma} \Lambda_{\mathrm{h}} \dot{\tilde{\gamma}},\left(\mathrm{H}_{\mathrm{h}} \mathrm{S}_{\mathrm{h}}-a\right) \dot{\theta}^{p}\right)_{h}= & -\frac{1}{2}\left(\frac{1}{\sigma} \Lambda_{\mathrm{h}} P_{M} \dot{\tilde{\gamma}},\left(\mathrm{H}_{\mathrm{h}} \mathrm{S}_{\mathrm{h}}-a\right) P_{M} \dot{\theta}^{p}\right)_{h} \\
& -\frac{1}{2}\left(\frac{1}{\sigma} \Lambda_{\mathrm{h}}\left(I-P_{M}\right) \dot{\tilde{\gamma}},\left(\mathrm{H}_{\mathrm{h}} \mathrm{S}_{\mathrm{h}}-a\right) P_{M} \dot{\theta}^{p}\right)_{h} \\
& -\frac{1}{2}\left(\frac{1}{\sigma} \Lambda_{\mathrm{h}} \dot{\tilde{\gamma}},\left(\mathrm{H}_{\mathrm{h}} \mathrm{S}_{\mathrm{h}}-a\right)\left(I-P_{M}\right) \dot{\theta}^{p}\right)_{h} .
\end{aligned}
$$

Fortunately, the terms involving $\left(I-P_{M}\right)$ are of low order. This is the content of the following lemma.

Lemma 7. Let $f(\cdot)$ and $g(\cdot) \in C^{2}[-\pi, \pi]$ and $\phi, \psi \in l^{2}$, then

$$
\begin{aligned}
\left|\left(f \Lambda_{\mathrm{h}}\left(I-P_{M}\right) \phi,\left(\mathrm{H}_{\mathrm{h}} \mathrm{S}_{\mathrm{h}}-a\right) P_{M} \psi\right)_{h}\right| & \leq C\|\phi\|_{l^{2}}\|\psi\|_{l^{2}}, \\
\left|\left(f \Lambda_{\mathrm{h}} \phi,\left(\mathrm{H}_{\mathrm{h}} \mathrm{S}_{\mathrm{h}}-a\right)\left(I-P_{M}\right) \psi\right)_{h}\right| & \leq C\|\phi\|_{l^{2}}\|\psi\|_{l^{2}}, \\
\left|\left(f \mathrm{D}_{\mathrm{h}}\left(I-P_{M}\right) \phi,\left(\mathrm{H}_{\mathrm{h}} \mathrm{S}_{\mathrm{h}}-a\right) \psi\right)_{h}\right| & \leq C\|\phi\|_{l^{2}}\|\psi\|_{l^{2}}, \\
\left|\left(f \mathrm{D}_{\mathrm{h}} \phi,\left(\mathrm{H}_{\mathrm{h}} \mathrm{S}_{\mathrm{h}}-a\right)\left(I-P_{M}\right) \psi\right)_{h}\right| & \leq C\|\phi\|_{l^{2}}\|\psi\|_{l^{2}} .
\end{aligned}
$$


Therefore, using parts (i) and (ii) of this result, and estimates (81) and (82), we conclude that

$$
\begin{aligned}
\frac{1}{4 S} \frac{d}{d t}\left(\sigma, \dot{\tilde{\gamma}}^{2}\right)_{h} & \leq-\frac{1}{2}\left(\frac{1}{\sigma} \Lambda_{\mathrm{h}} P_{M} \dot{\tilde{\gamma}},\left(\mathrm{H}_{\mathrm{h}} \mathrm{S}_{\mathrm{h}}-a\right) P_{M} \dot{\theta}^{p}\right)_{h} \\
& +C\left(\|\dot{\tilde{\gamma}}\|_{l^{2}}^{2}+\|\dot{\sigma}\|_{l^{2}}^{2}+\|\dot{\theta}\|_{l^{2}}^{2}+O\left(h^{m}\right)\|\dot{\tilde{\gamma}}\|_{l^{2}}\right)
\end{aligned}
$$

It is now clear that to balance the leading order term of this estimate, we need to take the inner product of $(78)$ and $\left(\mathrm{H}_{\mathrm{h}} \mathrm{S}_{\mathrm{h}}-a\right) P_{M} \dot{\theta}^{p}$. Note that $\left(\mathrm{H}_{\mathrm{h}} \mathrm{S}_{\mathrm{h}}-a\right) P_{M}$ is a positive operator; we will deal with the low modes of $\dot{\theta}$ later on. We have

$$
\begin{aligned}
\left(\left(\mathrm{H}_{\mathrm{h}} \mathrm{S}_{\mathrm{h}}-a\right) P_{M} \dot{\theta}^{p}, \frac{d \dot{\theta}}{d t}\right)_{h} & =\frac{1}{2}\left(\frac{1}{\sigma} \Lambda_{\mathrm{h}} \dot{\tilde{\gamma}},\left(\mathrm{H}_{\mathrm{h}} \mathrm{S}_{\mathrm{h}}-a\right) P_{M} \dot{\theta}^{p}\right)_{h} \\
& +\left(f \mathrm{D}_{\mathrm{h}} \dot{\theta},\left(\mathrm{H}_{\mathrm{h}} \mathrm{S}_{\mathrm{h}}-a\right) P_{M} \dot{\theta}^{p}\right)_{h} \\
& +\left(\Theta(\dot{\theta}),\left(\mathrm{H}_{\mathrm{h}} \mathrm{S}_{\mathrm{h}}-a\right) P_{M} \dot{\theta}^{p}\right)_{h} \\
& +\left(A_{-1}(\dot{\sigma}, \dot{\theta}, \dot{\tilde{\gamma}})+O\left(h^{m+1}\right),\left(\mathrm{H}_{\mathrm{h}} \mathrm{S}_{\mathrm{h}}-a\right) P_{M} \dot{\theta}^{p}\right)_{h},
\end{aligned}
$$

where $\Theta(\dot{\theta})$ is of the form defined in (80). Let us start with the left hand side of this equation,

$$
\begin{aligned}
\left(\left(\mathrm{H}_{\mathrm{h}} \mathrm{S}_{\mathrm{h}}-a\right) P_{M} \dot{\theta}, \frac{d \dot{\theta}}{d t}\right)_{h} & =\left(\left(\mathrm{H}_{\mathrm{h}} \mathrm{S}_{\mathrm{h}}-a\right) P_{M} \dot{\theta}, \frac{d}{d t} P_{M} \dot{\theta}\right)_{h} \\
& +\left(\left(\mathrm{H}_{\mathrm{h}} \mathrm{S}_{\mathrm{h}}-a\right) P_{M} \dot{\theta},\left(I-P_{M}\right) \frac{d \dot{\theta}}{d t}\right)_{h}
\end{aligned}
$$

However, we also have the following orthogonality property:

$$
\left(\Lambda_{\mathrm{h}} P_{M} \dot{\theta},\left(I-P_{M}\right) \frac{d \dot{\theta}}{d t}\right)_{h} \equiv 0 \text {. }
$$

Thus we get,

$$
\begin{aligned}
& \left(\left(\mathrm{H}_{\mathrm{h}} \mathrm{S}_{\mathrm{h}}-a\right) P_{M} \dot{\theta}^{p},\left(I-P_{M}\right) \frac{d \dot{\theta}}{d t}\right)_{h}=-\left(a P_{M} \dot{\theta}^{p},\left(I-P_{M}\right) \frac{d \dot{\theta}}{d t}\right)_{h} \\
& =-\left(a P_{M} \dot{\theta}^{p},\left(I-P_{M}\right)\left[\frac{1}{2 \sigma} \Lambda_{\mathrm{h}} \dot{\tilde{\gamma}}+f \mathrm{D}_{\mathrm{h}} \dot{\theta}+A_{0}(\dot{\sigma}, \dot{\theta})+A_{-1}(\dot{\tilde{\gamma}})+O\left(h^{m+1}\right)\right]\right)_{h},
\end{aligned}
$$

and using Lemma 7, it follows that

$$
\left(\left(\mathrm{H}_{\mathrm{h}} \mathrm{S}_{\mathrm{h}}-a\right) P_{M} \dot{\theta}^{p}, \frac{d \dot{\theta}}{d t}\right)_{h}=\frac{1}{2} \frac{d}{d t}\left(\left(\mathrm{H}_{\mathrm{h}} \mathrm{S}_{\mathrm{h}}-a\right) P_{M} \dot{\theta}^{p}, P_{M} \dot{\theta}\right)_{h}+R
$$

where

$$
|R| \leq C\|\dot{\theta}\|_{l^{2}}\left(\|\dot{\sigma}\|_{l^{2}}+\|\dot{\theta}\|_{l^{2}}+\|\dot{\tilde{\gamma}}\|_{l^{2}}+h^{m}\right)
$$


We turn our attention now to the right hand side of (84). For the first term, we have

$$
\begin{aligned}
\frac{1}{2}\left(\frac{1}{\sigma} \Lambda_{\mathrm{h}} \dot{\tilde{\gamma}},\left(\mathrm{H}_{\mathrm{h}} \mathrm{S}_{\mathrm{h}}-a\right) P_{M} \dot{\theta}^{p}\right)_{h} & =\frac{1}{2}\left(\frac{1}{\sigma} \Lambda_{\mathrm{h}} P_{M} \dot{\tilde{\gamma}},\left(\mathrm{H}_{\mathrm{h}} \mathrm{S}_{\mathrm{h}}-a\right) P_{M} \dot{\theta}^{p}\right)_{h} \\
& +\frac{1}{2}\left(\frac{1}{\sigma} \Lambda_{\mathrm{h}}\left(I-P_{M}\right) \dot{\tilde{\gamma}},\left(\mathrm{H}_{\mathrm{h}} \mathrm{S}_{\mathrm{h}}-a\right) P_{M} \dot{\theta}^{p}\right)_{h},
\end{aligned}
$$

and in view of Lemma 7 ,

$$
\begin{aligned}
\frac{1}{2}\left(\frac{1}{\sigma} \Lambda_{\mathrm{h}} \dot{\tilde{\gamma}},\left(\mathrm{H}_{\mathrm{h}} \mathrm{S}_{\mathrm{h}}-a\right) P_{M} \dot{\theta}^{p}\right)_{h} & \leq \frac{1}{2}\left(\frac{1}{\sigma} \Lambda_{\mathrm{h}} P_{M} \dot{\tilde{\gamma}},\left(\mathrm{H}_{\mathrm{h}} \mathrm{S}_{\mathrm{h}}-a\right) P_{M} \dot{\theta}^{p}\right)_{h} \\
& +C\|\dot{\theta}\|_{l^{2}}\|\dot{\tilde{\gamma}}\|_{l^{2}} .
\end{aligned}
$$

Similarly, separating low and high modes, we have that

$$
\left(f \mathrm{D}_{\mathrm{h}} \dot{\theta},\left(\mathrm{H}_{\mathrm{h}} \mathrm{S}_{\mathrm{h}}-a\right) P_{M} \dot{\theta}^{p}\right)_{h} \leq\left(f \mathrm{D}_{\mathrm{h}} P_{M} \dot{\theta},\left(\mathrm{H}_{\mathrm{h}} \mathrm{S}_{\mathrm{h}}-a\right) P_{M} \dot{\theta}^{p}\right)_{h}+C\|\dot{\theta}\|_{l^{2}}^{2},
$$

but for any $l^{2}$ function $\phi$,

$$
\begin{aligned}
\left(f \mathrm{D}_{\mathrm{h}} \phi, \Lambda_{\mathrm{h}} \phi\right)_{h} & =-\left(\mathrm{H}_{\mathrm{h}}\left(f \mathrm{D}_{\mathrm{h}} \phi\right), \mathrm{D}_{\mathrm{h}} \phi\right)_{h} \\
& =-\left(\mathrm{H}_{\mathrm{h}} \mathrm{D}_{\mathrm{h}} \phi, f \mathrm{D}_{\mathrm{h}} \phi\right)_{h}+\left(A_{-1}(\phi), \mathrm{D}_{\mathrm{h}} \phi\right)_{h} .
\end{aligned}
$$

Thus,

$$
\left(f \mathrm{D}_{\mathrm{h}} \phi, \Lambda_{\mathrm{h}} \phi\right)_{h}=\left(A_{0}(\phi), \phi\right)_{h} .
$$

On the other hand, we have already shown that $\left|\left(f \phi, \mathrm{D}_{\mathrm{h}} \phi\right)_{h}\right| \leq C\|\phi\|_{l^{2}}^{2}$ (see (82)). Therefore,

$$
\left(f \mathrm{D}_{\mathrm{h}} P_{M} \dot{\theta},\left(\mathrm{H}_{\mathrm{h}} \mathrm{S}_{\mathrm{h}}-a\right) P_{M} \dot{\theta}^{p}\right)_{h} \leq C\|\dot{\theta}\|_{H^{1 / 2}}^{2} .
$$

The following result will help us estimate the term containing $\Theta(\dot{\theta})$ in (84), (see $[15])$.

Lemma 8. Let $f(\cdot) \in C^{2}$, then

$$
\begin{aligned}
&\left|\left(f \phi, \mathrm{H}_{\mathrm{h}} \mathrm{S}_{\mathrm{h}} \psi\right)_{h}\right| \leq C\|\phi\|_{H_{h}^{1 / 2}}\|\psi\|_{H_{h}^{1 / 2}}, \\
&\left|\left(f \mathrm{H}_{\mathrm{h}} \phi, \mathrm{H}_{\mathrm{h}} \mathrm{S}_{\mathrm{h}} \psi\right)_{h}\right| \leq C\|\phi\|_{H_{h}^{1 / 2}}\|\psi\|_{H_{h}^{1 / 2}}, \\
&\left|\left(f \mathrm{~S}_{\mathrm{h}} \mathrm{G}_{\mathrm{h}}^{\mathrm{p}} \phi, \mathrm{H}_{\mathrm{h}} \mathrm{S}_{\mathrm{h}} \psi\right)_{h}\right| \leq C\|\phi\|_{H_{h}^{1 / 2}}\|\psi\|_{H_{h}^{1 / 2}}, \\
&\left|\left(f \mathrm{~S}_{\mathrm{h}} g \mathrm{G}_{\mathrm{h}}^{\mathrm{p}} \mathrm{H}_{\mathrm{h}} \phi, \mathrm{H}_{\mathrm{h}} \mathrm{S}_{\mathrm{h}} \psi\right)_{h}\right| \leq C\|\phi\|_{H_{h}^{1 / 2}}\|\psi\|_{H_{h}^{1 / 2}} .
\end{aligned}
$$

where $\|\phi\|_{H_{h}^{1 / 2}}^{2} \equiv\left(\left(\Lambda_{\mathrm{h}}+I\right) \phi, \phi\right)_{h}$ and $\Lambda_{\mathrm{h}}=\mathrm{H}_{\mathrm{h}} \mathrm{D}_{\mathrm{h}}$.

Note that all the first entries in the discrete inner products, i.e., $f \phi, f \mathrm{H}_{\mathrm{h}} \phi$, $f \mathrm{~S}_{\mathrm{h}} \mathrm{G}_{\mathrm{h}}^{\mathrm{p}} \phi$, etc., are of order $\phi$ which makes clear why this result is true.

For the $\Theta$ term in (84), we have

$$
\left|\left(\Theta(\dot{\theta}),\left(\mathrm{H}_{\mathrm{h}} \mathrm{S}_{\mathrm{h}}-a\right) P_{M} \dot{\theta}^{p}\right)_{h}\right| \leq C\|\dot{\theta}\|_{H_{h}^{1 / 2}}^{2}+\left|\left(f \mathrm{~S}_{\mathrm{h}} E(\dot{\theta}),\left(\mathrm{H}_{\mathrm{h}} \mathrm{S}_{\mathrm{h}}-a\right) P_{M} \dot{\theta}^{p}\right)_{h}\right| .
$$

Recall that the operator $E(\dot{\theta})$ has the following general form,

$$
E\left(\dot{\theta}_{i}\right)=f\left(\alpha_{i}\right) \operatorname{Int}_{\mathrm{h}}\left(z_{\alpha} \dot{\theta}\right)_{i}^{p}+g\left(\alpha_{i}\right) \mathrm{H}_{\mathrm{h}} \operatorname{Int}_{\mathrm{h}}\left(z_{\alpha} \dot{\theta}\right)_{i}^{p} .
$$


Also note that

$$
\begin{aligned}
\mathrm{S}_{\mathrm{h}}\left(f\left(\alpha_{i}\right) \operatorname{Int}_{\mathrm{h}}\left(z_{\alpha} \dot{\theta}\right)_{i}^{p}\right) & =\mathrm{D}_{\mathrm{h}}\left(f\left(\alpha_{i}\right) \operatorname{Int}_{\mathrm{h}}\left(z_{\alpha} \dot{\theta}\right)_{i}\right)+\mathrm{S}_{\mathrm{h}} \mathrm{G}_{\mathrm{h}}^{\mathrm{p}} \operatorname{Int}_{\mathrm{h}}\left(z_{\alpha} \dot{\theta}\right)_{i} \\
& =f\left(\alpha_{i}\right) \dot{\theta}^{p}+f_{\alpha}\left(\alpha_{i}\right) \tilde{\dot{\theta}}_{i}^{q}+\mathrm{S}_{\mathrm{h}} \mathrm{G}_{\mathrm{h}}^{\mathrm{p}} \tilde{\dot{\theta}}_{i}+A_{-1}\left(\dot{\theta}_{i}\right),
\end{aligned}
$$

where $\tilde{\dot{\theta}}_{i} \equiv \operatorname{Int}_{\mathrm{h}}\left(z_{\alpha} \dot{\theta}\right)_{i}$, and similarly

$$
\mathrm{S}_{\mathrm{h}}\left(g\left(\alpha_{i}\right) \mathrm{H}_{\mathrm{h}} \operatorname{Int}_{\mathrm{h}}\left(z_{\alpha} \dot{\theta}\right)_{i}^{p}\right)=g\left(\alpha_{i}\right) \mathrm{H}_{\mathrm{h}} \dot{\theta}^{p}+g_{\alpha}\left(\alpha_{i}\right) \mathrm{H}_{\mathrm{h}} \tilde{\dot{\theta}}_{i}^{q}+\mathrm{S}_{\mathrm{h}} \mathrm{G}_{\mathrm{h}}^{\mathrm{p}} \mathrm{H}_{\mathrm{h}} \tilde{\dot{\theta}}_{i}+A_{-1}\left(\dot{\theta}_{i}\right) .
$$

Moreover, $\|\tilde{\dot{\theta}}\|_{h_{h}^{1 / 2}}^{2} \leq C\|\dot{\theta}\|_{H_{h}^{1 / 2}}^{2}$. Therefore, using Lemma 8, we get

$$
\left|\left(\Theta(\dot{\theta}),\left(\mathrm{H}_{\mathrm{h}} \mathrm{S}_{\mathrm{h}}-a\right) P_{M} \dot{\theta}^{p}\right)_{h}\right| \leq C\|\dot{\theta}\|_{H_{h}^{1 / 2}}^{2} .
$$

Finally, we clearly have

$$
\begin{array}{r}
\left|\left(A_{-1}(\dot{\sigma}, \dot{\theta}, \dot{\tilde{\gamma}})+O\left(h^{m+1}\right),\left(\mathrm{H}_{\mathrm{h}} \mathrm{S}_{\mathrm{h}}-a\right) P_{M} \dot{\theta}^{p}\right)_{h}\right| \\
\leq C\|\dot{\theta}\|_{l^{2}}\left(\|\dot{\theta}\|_{l^{2}}+\|\dot{\sigma}\|_{l^{2}}+\|\dot{\tilde{\gamma}}\|_{l^{2}}+h^{m}\right) .
\end{array}
$$

Therefore, we conclude that

$$
\begin{aligned}
\frac{1}{2} \frac{d}{d t}\left(\left(\mathrm{H}_{\mathrm{h}} \mathrm{S}_{\mathrm{h}}-a\right) P_{M} \dot{\theta}^{p}, P_{M} \dot{\theta}\right)_{h} \leq & \frac{1}{2}\left(\frac{1}{\sigma} \Lambda_{\mathrm{h}} P_{M} \dot{\tilde{\gamma}},\left(\mathrm{H}_{\mathrm{h}} \mathrm{S}_{\mathrm{h}}-a\right) P_{M} \dot{\theta}^{p}\right)_{h} \\
& +C\|\dot{\theta}\|_{H_{h}^{1 / 2}}\left(\|\dot{\theta}\|_{H_{h}^{1 / 2}}+\|\dot{\sigma}\|_{l^{2}}+\|\dot{\tilde{\gamma}}\|_{l^{2}}+h^{m}\right)
\end{aligned}
$$

Now taking the inner product of (77) and $\dot{\sigma}$, it follows immediately that

$$
\frac{1}{2} \frac{d}{d t}\|\dot{\sigma}\|_{l^{2}}^{2} \leq C\|\dot{\theta}\|_{l^{2}}\left(\|\dot{\theta}\|_{l^{2}}+\|\dot{\sigma}\|_{l^{2}}+\|\dot{\tilde{\gamma}}\|_{l^{2}}+h^{m+2}\right)
$$

We need to consider the contribution of the low modes to complete the energy estimates. Taking the inner product of the $\dot{\theta}$-equation (78) and $\left(I-P_{M}\right) \dot{\theta}$, we obtain

$$
\begin{aligned}
\left(\left(I-P_{M}\right) \dot{\theta}, \frac{d \dot{\theta}}{d t}\right)_{h} & =\frac{1}{2}\left(\frac{1}{\sigma} \Lambda_{\mathrm{h}} \dot{\tilde{\gamma}},\left(I-P_{M}\right) \dot{\theta}\right)_{h} \\
& +\left(f \mathrm{D}_{\mathrm{h}} \dot{\theta},\left(I-P_{M}\right) \dot{\theta}\right)_{h}+\left(\Theta(\dot{\theta}),\left(I-P_{M}\right) \dot{\theta}\right)_{h} \\
& +\left(A_{-1}(\dot{\sigma}, \dot{\theta}, \dot{\tilde{\gamma}})+O\left(h^{m+1}\right),\left(I-P_{M}\right) \dot{\theta}\right)_{h} .
\end{aligned}
$$

For the left hand side of this expression, we note that

$$
\left(\left(I-P_{M}\right) \dot{\theta}, \frac{d \dot{\theta}}{d t}\right)_{h}=\left(\left(I-P_{M}\right) \dot{\theta}, \frac{d}{d t}\left(I-P_{M}\right) \dot{\theta}\right)_{h}=\frac{1}{2} \frac{d}{d t}\left\|\left(I-P_{M}\right) \dot{\theta}\right\|_{l^{2}}^{2}
$$

where we have used the orthogonality relation

$$
\left(\left(I-P_{M}\right) \dot{\theta}, P_{M} \frac{d \dot{\theta}}{d t}\right)_{h} \equiv 0 .
$$

In view of Lemma 7 , and that $\Theta\left(\dot{\theta}_{i}\right)=A_{0}\left(\dot{\theta}_{i}\right)$, we conclude that

$$
\frac{1}{2} \frac{d}{d t}\left\|\left(I-P_{M}\right) \dot{\theta}\right\|_{l^{2}}^{2} \leq C\|\dot{\theta}\|_{l^{2}}\left(\|\dot{\theta}\|_{l^{2}}+\|\dot{\sigma}\|_{l^{2}}+\|\dot{\tilde{\gamma}}\|_{l^{2}}+h^{m+1}\right) .
$$


We put together now the two bands of modes by noting that

$$
\begin{aligned}
& \left(\left(\mathrm{H}_{\mathrm{h}} \mathrm{S}_{\mathrm{h}}-a\right) P_{M} \dot{\theta}^{p}, P_{M} \dot{\theta}\right)_{h}+\frac{M}{2}\left\|\left(I-P_{M}\right) \dot{\theta}\right\|_{l^{2}}^{2} \\
& =\left(\Lambda_{\mathrm{h}} P_{M} \dot{\theta}, P_{M} \dot{\theta}\right)_{h}-\left(a P_{M} \dot{\theta}^{p}, P_{M} \dot{\theta}\right)_{h}+\frac{M}{2}\left\|\left(I-P_{M}\right) \dot{\theta}\right\|_{l^{2}}^{2} \\
& \quad \geq\left(\Lambda_{\mathrm{h}} P_{M} \dot{\theta}, P_{M} \dot{\theta}\right)_{h}-\|a\|_{\infty}\left\|P_{M} \dot{\theta}^{p}\right\|_{l^{2}}^{2}+\frac{M}{2}\left\|\left(I-P_{M}\right) \dot{\theta}\right\|_{l^{2}}^{2} \\
& =2 \pi \sum_{\substack{|k|>M \\
k \in I}} \rho(k h)\left[|k|-\|a\|_{\infty}\right]\left|\hat{\dot{\theta}}_{k}\right|^{2}+2 \pi \frac{M}{2} \sum_{\substack{|k| \leq M \\
k \in I}}\left|\hat{\dot{\theta}}_{k}\right|^{2},
\end{aligned}
$$

where $I=\{-N / 2+1, \ldots, N / 2\}$. Recall that $M=2\left(\|a\|_{\infty}+1\right)$. Therefore,

$$
\frac{|k|}{2}>\|a\|_{\infty} \text { for }|k| \geq M
$$

and as a result,

$$
\begin{aligned}
& \left(\left(\mathrm{H}_{\mathrm{h}} \mathrm{S}_{\mathrm{h}}-a\right) P_{M} \dot{\theta}^{p}, P_{M} \dot{\theta}\right)_{h}+\frac{M}{2}\left\|\left(I-P_{M}\right) \dot{\theta}\right\|_{l^{2}}^{2} \\
& \geq 2 \pi\left[\frac{1}{2} \sum_{\substack{|k|>M \\
k \in I}} \rho(k h)|k|\left|\hat{\dot{\theta}}_{k}\right|^{2}+\frac{M}{2} \sum_{\substack{|k| \leq M \\
k \in I}}\left|\hat{\dot{\theta}}_{k}\right|^{2}\right] \\
& =\frac{\pi}{2}\left[\sum_{\substack{|k|>M \\
k \in I}} \rho(k h)|k|\left|\hat{\dot{\theta}}_{k}\right|^{2}+M \sum_{\substack{|k| \leq M \\
k \in I}}\left|\hat{\dot{\theta}}_{k}\right|^{2}\right] \\
& \quad+\frac{\pi}{2}\left[\sum_{\substack{|k|>M \\
k \in I}} \rho(k h)|k|\left|\hat{\theta}_{k}\right|^{2}+M \sum_{\substack{|k| \leq M \\
k \in I}}\left|\hat{\dot{\theta}}_{k}\right|^{2}\right] \geq \frac{1}{4}\|\dot{\theta}\|_{H_{h}^{1 / 2}}^{2} .
\end{aligned}
$$

We now add the estimates (83), (88), (89), and $M / 2$ times the low-mode estimate (90). As noted before, the leading order terms of (83) and (88) cancel each other, and we are left with the following inequality,

$$
\begin{aligned}
\frac{1}{4 S} \frac{d}{d t} & \left(\sigma, \dot{\tilde{\gamma}}^{2}\right)_{h}+\frac{1}{2} \frac{d}{d t}\|\dot{\sigma}\|_{l^{2}}^{2}+\frac{1}{2} \frac{d}{d t}\left(\left(\mathrm{H}_{\mathrm{h}} \mathrm{S}_{\mathrm{h}}-a\right) P_{M} \dot{\theta}^{p}, P_{M} \dot{\theta}\right)_{h} \\
& +\frac{M}{2} \frac{d}{d t}\left\|\left(I-P_{M}\right) \dot{\theta}\right\|_{l^{2}}^{2} \\
& \leq C\left(\|\dot{\theta}\|_{H_{h}^{1 / 2}}^{2}+\|\dot{\tilde{\gamma}}\|_{l^{2}}^{2}+\|\dot{\sigma}\|_{l^{2}}^{2}\right)+C\left(\|\dot{\theta}\|_{H_{h}^{1 / 2}}+\|\dot{\tilde{\gamma}}\|_{l^{2}}+\|\dot{\sigma}\|_{l^{2}}\right) h^{m} .
\end{aligned}
$$

Integrating in time, we find that

where

$$
y_{0}^{2}(t) \leq y_{0}^{2}(0)+\int_{0}^{t} K\left(y_{0}^{2}(s)+y_{0}(s) h^{m}\right) d s,
$$

$$
y_{0}^{2}(t)=\|\dot{\theta}\|_{H_{h}^{1 / 2}}^{2}+\|\dot{\tilde{\gamma}}\|_{l^{2}}^{2}+\|\dot{\sigma}\|_{l^{2}}^{2} .
$$

Applying Gronwall's inequality, we conclude that

$$
y_{0}(t) \leq C(T) h^{m} \quad \text { for } \quad t \leq T^{*} .
$$

Therefore,

$$
\|\dot{\sigma}\|_{l^{2}},\|\dot{\tilde{\gamma}}\|_{l^{2}},\|\dot{\theta}\|_{H_{h}^{1 / 2}} \leq C(T) h^{m}
$$


On the other hand from the evolution equation (79) for $\dot{\tilde{\gamma}}$, we have that for $t \leq T^{*}$,

$$
\left\|\frac{d \dot{\tilde{\gamma}}}{d t}\right\|_{l^{2}} \leq \frac{C}{h^{2}}\left(\|\dot{\sigma}\|_{l^{2}}+\|\dot{\theta}\|_{l^{2}}+\|\dot{\tilde{\gamma}}\|_{l^{2}}\right) \leq \tilde{C}(T) h^{m-3} .
$$

Since $m>4$, we get that for small $h$,

$$
\|\dot{\sigma}\|_{l^{2}},\|\dot{\tilde{\gamma}}\|_{l^{2}} \leq C(T) h^{5}<\frac{1}{2} h^{7 / 2}
$$

and

$$
\|\dot{\theta}\|_{l^{2}} \leq B(T) h^{4}<\frac{1}{2} h^{7 / 2} \quad \text { and } \quad\left\|\frac{d \dot{\tilde{\gamma}}}{d t}\right\|_{l^{2}}<\frac{1}{2} h^{3 / 2} .
$$

From the definition (42) of $T^{*}$, it follows that

$$
T=T^{*},
$$

which shows that the estimates (91) are valid for the entire interval $0 \leq t \leq T$. This completes the proof of Theorem 1 for the convergence of our method.

\section{TIME DISCRETE ANALYSIS FOR A SIMPLE MODEL}

The study of the fully discrete scheme for our two-fluid method is awfully technical. Even a frozen coefficient analysis for the vortex sheet case $(A=0)$ is complicated.

To illustrate how the high order stiffness is removed and what the linear time step constraint is to achieve numerical stability, we present here a complete stability analysis for the simplest case of Hele-Shaw interface (fluids of same viscosity and density).

The interface position is again determined by the same evolution equations for $\sigma$ and $\theta$, but now $\gamma$ is a dependent variable. For the simplest case, we have [24]

$$
\gamma=S\left(\frac{\theta_{\alpha}}{\sigma}\right)_{\alpha}
$$

Using the small scale decomposition in the evolution equation for $\theta$ and (93), we now consider the following Crank-Nicholson discretization in time,

$$
\frac{\theta_{i}^{n+1}-\theta_{i}^{n-1}}{2 \Delta t}=\frac{S}{4\left(\sigma^{n}\right)^{3}} \mathrm{H}_{\mathrm{h}} \mathrm{D}_{\mathrm{h}}{ }^{3}\left(\theta_{i}^{n+1}+\theta_{i}^{n-1}\right)+P_{i},
$$

where $P_{i}$ is the right hand side of $(26)$ minus the first term in the right hand side of (94). We are also taking $R \equiv 1$, that is, an equal arclength frame. To compute the velocity, $\gamma$ is determined from $\gamma_{i}^{n}=S \mathrm{D}_{\mathrm{h}} \theta_{i}^{n} / \sigma^{n}$. As we mentioned before, the equation for $\sigma$ is not stiff. We discretize it with a second order Adams-Bashforth scheme,

$$
\sigma^{n+1}=\sigma^{n}-\Delta t\left(\frac{3}{2}\left\langle\mathrm{~S}_{\mathrm{h}} \theta^{n} U^{N, n}\right\rangle_{h}-\frac{1}{2}\left\langle S h_{\theta}^{n-1} U^{N, n-1}\right\rangle_{h}\right) .
$$

Assuming a relation of the form $\Delta t \leq C h$ (we aim to determine more precisely what the constant $\mathrm{C}$ should be), we would have an overall accuracy of $O\left(h^{2}\right)$. This accuracy is not high enough to bound the nonlinear terms as we did in the time continuous analysis. Nevertheless, an argument due to Strang [41] can be used to overcome this difficulty. The idea is to construct functions

$$
\begin{aligned}
& \tilde{\theta}(\alpha, t)=\theta(\alpha, t)+(\Delta t)^{2} \theta_{2}(\alpha, t), \\
& \tilde{\sigma}(\alpha, t)=\sigma(\alpha, t)+(\Delta t)^{2} \sigma_{2}(\alpha, t),
\end{aligned}
$$


which satisfy the scheme with a truncation error of order $O\left((\Delta t)^{4}+h^{m}\right)$, that is $O\left(h^{4}\right)$. This accuracy is just enough to bound the nonlinear terms. Hence, the same estimates as in the time continuous case can be applied to this fully discrete situation to obtain the following equations for the errors,

$$
\begin{aligned}
\frac{\dot{\theta}_{i}^{n+1}-\dot{\theta}_{i}^{n-1}}{2 \Delta t} & =\frac{S}{4\left(\sigma^{n}\right)^{3}} \mathrm{H}_{\mathrm{h}} \mathrm{D}_{\mathrm{h}}{ }^{3}\left(\dot{\theta}_{i}^{n+1}+\dot{\theta}_{i}^{n-1}\right)+\frac{U^{A}\left(\alpha_{i}, t_{n}\right)}{\sigma\left(t_{n}\right)} \mathrm{D}_{\mathrm{h}} \dot{\theta}_{i}^{n} \\
& +f\left(\alpha_{i}, t_{n}\right) \dot{\sigma}^{n}+A_{0}\left(\dot{\theta}_{i}^{n}\right)+O\left((\Delta t)^{2}+h^{m}\right), \\
\dot{\sigma}^{n+1}-\dot{\sigma}^{n} & =-\frac{1}{2} \Delta t\left(3 \dot{T}^{n}-\dot{T}^{n-1}\right)+\Delta t \cdot O\left((\Delta t)^{2}+h^{m}\right),
\end{aligned}
$$

where

$$
\left|\dot{T}^{k}\right| \leq C_{k}\left(\left\|\dot{\theta}^{k}\right\|_{l^{2}}+\left|\dot{\sigma}^{k}\right|\right)
$$

and $t_{n}=n \Delta t$. Multiply equation (97) by $\dot{\sigma}^{n+1}+\dot{\sigma}^{n}$ and sum in time to get

$$
\begin{array}{r}
\left(\dot{\sigma}^{n+1}\right)^{2}-\left(\dot{\sigma}^{0}\right)^{2}=-\frac{3}{2} \Delta t \sum_{k=0}^{n} \dot{T}^{k}\left(\dot{\sigma}^{k+1}+\dot{\sigma}^{k}\right)+\frac{1}{2} \Delta t \sum_{k=0}^{n} \dot{T}^{k-1}\left(\dot{\sigma}^{k+1}+\dot{\sigma}^{k}\right) \\
+\Delta t \sum_{k=0}^{n} O\left((\Delta t)^{2}+h^{m}\right)\left(\dot{\sigma}^{k+1}+\dot{\sigma}^{k}\right) .
\end{array}
$$

Without loss of generality, we shall assume that the error in the first two steps is zero. Thus, for $\Delta t$ small and using (98) we obtain

$$
\left(\dot{\sigma}^{n+1}\right)^{2} \leq C \Delta t \sum_{k=1}^{n}\left(\left\|\dot{\theta}^{k}\right\|_{l^{2}}^{2}+\left(\dot{\sigma}^{k}\right)^{2}\right)+C \Delta t\left((\Delta t)^{2}+h^{m}\right) \sum_{k=1}^{n}\left|\dot{\sigma}^{k}\right| .
$$

Now multiply equation (96) by $2 \Delta t \cdot h\left(\dot{\theta}_{i}^{n+1}+\dot{\theta}_{i}^{n-1}\right)$, and sum in space and time to obtain

$$
\begin{aligned}
\left\|\dot{\theta}^{n+1}\right\|_{l^{2}}^{2}+\left\|\dot{\theta}^{n}\right\|_{l^{2}}^{2}= & \frac{1}{2} S \Delta t \sum_{k=1}^{n} \frac{1}{\sigma^{3}\left(t_{k}\right)}\left(\dot{\theta}^{k+1}+\dot{\theta}^{k-1}, \mathrm{H}_{\mathrm{h}} \mathrm{D}_{\mathrm{h}}{ }^{3}\left(\dot{\theta}^{k+1}+\dot{\theta}^{k-1}\right)\right)_{h} \\
& +2 \Delta t \sum_{k=1}^{n} \frac{1}{\sigma\left(t_{k}\right)}\left(\dot{\theta}^{k+1}+\dot{\theta}^{k-1}, U^{A}\left(\cdot, t_{k}\right) \mathrm{D}_{\mathrm{h}} \dot{\theta}^{k}\right)_{h} \\
& +2 \Delta t \sum_{k=1}^{n} \dot{\sigma}^{k}\left(\dot{\theta}^{k+1}+\dot{\theta}^{k-1}, f\left(\cdot, t_{k}\right)\right)_{h} \\
& +2 \Delta t \sum_{k=1}^{n}\left(\dot{\theta}^{k+1}+\dot{\theta}^{k-1}, A_{0}\left(\dot{\theta}^{k}\right)+O\left((\Delta t)^{2}+h^{m}\right)\right)_{h} \\
= & (\mathrm{I})+(\mathrm{II})+(\mathrm{III})+(\mathrm{IV}) .
\end{aligned}
$$

Now, note that $\left(\dot{\phi}, \mathrm{H}_{\mathrm{h}} \mathrm{D}_{\mathrm{h}}{ }^{3} \dot{\phi}\right)_{h} \leq 0$. Therefore $(\mathrm{I}) \leq 0$. For the second term, we have

$$
\begin{aligned}
(\mathrm{II})=2 \Delta & t \sum_{k=1}^{n} \frac{1}{\sigma\left(t_{k}\right)}\left(\dot{\theta}^{k+1}, U^{A}\left(\cdot, t_{k}\right) \mathrm{D}_{\mathrm{h}} \dot{\theta}^{k}\right)_{h} \\
& +2 \Delta t \sum_{k=1}^{n} \frac{1}{\sigma\left(t_{k}\right)}\left(\dot{\theta}^{k-1}, U^{A}\left(\cdot, t_{k}\right) \mathrm{D}_{\mathrm{h}} \dot{\theta}^{k}\right)_{h},
\end{aligned}
$$


but

$$
\begin{aligned}
2 \Delta t \sum_{k=1}^{n} & \frac{1}{\sigma\left(t_{k}\right)}\left(\dot{\theta}^{k-1}, U^{A}\left(\cdot, t_{k}\right) \mathrm{D}_{\mathrm{h}} \dot{\theta}^{k}\right)_{h} \\
= & -2 \Delta t \sum_{k=1}^{n} \frac{1}{\sigma\left(t_{k}\right)}\left(\dot{\theta}^{k}, U^{A}\left(\cdot, t_{k-1}\right) \mathrm{D}_{\mathrm{h}} \dot{\theta}^{k-1}\right)_{h} \\
& +\Delta t \sum_{k=1}^{n} \frac{1}{\sigma\left(t_{k}\right)}\left(A_{0}\left(\dot{\theta}^{k-1}\right), \dot{\theta}^{k}\right)_{h},
\end{aligned}
$$

where we have summed by parts and shifted $U^{A}\left(\cdot, t_{k}\right)$ to $U^{A}\left(\cdot, t_{k-1}\right)$. Thus, the leading order terms of (II) telescope; we get

$$
(\mathrm{II})=2 \Delta t \frac{1}{\sigma\left(t_{n}\right)}\left(\dot{\theta}^{n+1}, U^{A}\left(\cdot, t_{n}\right) \mathrm{D}_{\mathrm{h}} \dot{\theta}^{n}\right)_{h}+\Delta t \sum_{k=1}^{n}\left(A_{0}\left(\dot{\theta}^{k-1}\right), \dot{\theta}^{k}\right)_{h} .
$$

Noting that

$$
2 \Delta t \frac{1}{\sigma\left(t_{n}\right)}\left(\dot{\theta}^{n+1}, U^{A}\left(\cdot, t_{n}\right) \mathrm{D}_{\mathrm{h}} \dot{\theta}^{n}\right)_{h} \leq \lambda_{n}\left(\left\|\dot{\theta}^{n+1}\right\|_{l^{2}}^{2}+\left\|\dot{\theta}^{n}\right\|_{l^{2}}^{2}\right)
$$

where we have bounded $\left\|\mathrm{D}_{\mathrm{h}} \dot{\theta}\right\|_{l^{2}}$ by $\|\dot{\theta}\|_{l^{2}} / h$ and absorbed the factor $1 / h$ in the following definition of $\lambda_{n}$,

$$
\lambda_{n} \equiv \frac{\Delta t}{h}\left\|\frac{U^{A}\left(t_{n}\right)}{\sigma\left(t_{n}\right)}\right\|_{\infty}
$$

we have that

$$
(\mathrm{II}) \leq \lambda_{n}\left(\left\|\dot{\theta}^{n+1}\right\|_{l^{2}}^{2}+\left\|\dot{\theta}^{n}\right\|_{l^{2}}^{2}\right)+C \Delta t \sum_{k=1}^{n}\left(\left\|\dot{\theta}^{k}\right\|_{l^{2}}^{2}+\left\|\dot{\theta}^{k-1}\right\|_{l^{2}}^{2}\right) .
$$

Now, for the third term we have

$$
|(\mathrm{III})| \leq C \Delta t \sum_{k=1}^{n}\left(\left\|\dot{\theta}^{k+1}\right\|_{l^{2}}^{2}+\left\|\dot{\theta}^{k-1}\right\|_{l^{2}}^{2}+\left(\dot{\sigma}^{k}\right)^{2}\right) .
$$

The fourth term is easily estimated;

$$
\begin{aligned}
|(\mathrm{IV})| \leq & C \Delta t \sum_{k=1}^{n}\left(\left\|\dot{\theta}^{k+1}\right\|_{l^{2}}^{2}+\left\|\dot{\theta}^{k}\right\|_{l^{2}}^{2}+\left\|\dot{\theta}^{k-1}\right\|_{l^{2}}^{2}\right) \\
& +C \Delta t\left((\Delta t)^{2}+h^{m}\right) \sum_{k=1}^{n}\left(\left\|\dot{\theta}^{k+1}\right\|_{l^{2}}^{2}+\left\|\dot{\theta}^{k-1}\right\|_{l^{2}}^{2} .\right.
\end{aligned}
$$

Collecting our estimates and assuming that $\lambda_{n}<1$, we can conclude that

$$
\varepsilon_{n+1}^{2} \leq C \Delta t\left[\sum_{k=1}^{n} \varepsilon_{k}^{2}+\left((\Delta t)^{2}+h^{m}\right) \varepsilon_{k}\right],
$$

where we have defined

$$
\varepsilon_{n+1}^{2} \equiv\left\|\dot{\theta}^{n+1}\right\|_{l^{2}}^{2}+\left\|\dot{\theta}^{n}\right\|_{l^{2}}^{2}+\left(\dot{\sigma}^{n}\right)^{2}
$$

which implies that

$$
\left\|\dot{\theta}^{n}\right\|_{l^{2}},\left|\dot{\sigma}^{n}\right| \leq C(T)\left((\Delta t)^{2}+h^{m}\right)
$$


Therefore the stability constraint is

$$
\Delta t \leq\left\|\frac{U^{A}\left(t_{n}\right)}{\sigma\left(t_{n}\right)}\right\|_{\infty}^{-1} h .
$$

Remark. From (59) and (63) we get that the leading order behavior of $U^{A}$ is

$$
U^{A} \sim \frac{1}{2} \tilde{\gamma}\left(\alpha_{i}\right) \mathrm{H}_{\mathrm{h}} \theta_{i},
$$

and since $\tilde{\gamma}$ is linked to the derivative of the curvature (in this case $\tilde{\gamma}=S \kappa_{\alpha} / \sigma$ ), the time step has to be reduced significantly whenever singular regions or high curvatures are encountered. This was observed in our numerics.

\section{NumericAL EXAMPLES}

We present in this section some numerical simulations of two fluid interfaces with surface tension, using the scheme studied in this paper. The examples illustrate the nonlinear stability of our discretization and robustness of our method.

The time integration is performed following the ideas of HLS to remove the high order stiffness introduced by surface tension; we rewrite the spectral discretization in space of the evolution equations for $\theta$ and $\gamma$, extracting the high mode leading order terms. The resulting system of ODEs can be written as

$$
\begin{aligned}
\frac{d \theta_{i}}{d t} & =\frac{1}{2 \sigma_{i}} \mathrm{~S}_{\mathrm{h}}\left(\frac{1}{\sigma_{i}} \mathrm{H}_{\mathrm{h}} \gamma_{i}^{p}\right)+P_{i}, \\
\frac{d \gamma_{i}}{d t} & =S \mathrm{D}_{\mathrm{h}}\left(\frac{1}{\sigma_{i}} \mathrm{D}_{\mathrm{h}} \theta_{i}\right)+Q_{i},
\end{aligned}
$$

where $P_{i}$ is the right hand side of (26) minus the first term in the right hand side of (105) and $Q_{i}$ is the right hand side of (27) without the surface tension term. Now we use a fourth order accurate implicit-explicit scheme studied by Ascher, Ruuth, and Wetton [1]. The scheme is suitable for time-dependent PDEs whose spatial derivatives have been discretized by central finite differences or by some spectral method, giving rise to a large system of ODEs of the form

$$
\frac{d u}{d t}=f(u)+\nu g(u)
$$

where $g$ is a linear operator containing derivatives of high order, $f$ is a collection of nonlinear terms which we prefer not to integrate implicitly, and $\nu$ is a positive parameter. Typically $f$ contains only first order derivatives, thus the main stiffness is introduced by the linear operator $g$. The implicit-explicit time integration scheme is given by

$$
\begin{aligned}
& \frac{1}{\Delta t}\left(\frac{25}{12} u^{n+1}-4 u^{n}+3 u^{n-1}-\frac{4}{3} u^{n-2}+\frac{1}{4} u^{n-3}\right) \\
& \quad=4 f\left(u^{n}\right)-6 f\left(u^{n-1}\right)+4 f\left(u^{n-2}\right)-f\left(u^{n-3}\right)+\nu g\left(u^{n+1}\right) .
\end{aligned}
$$

Applying this scheme to (105)-(106), we obtain the following system of equations

$$
\begin{aligned}
\theta_{i}^{n+1}-\frac{\Delta t}{2} \frac{12}{25} \frac{1}{\sigma_{i}^{n+1}} \mathrm{~S}_{\mathrm{h}}\left(\frac{1}{\sigma_{i}^{n+1}} \mathrm{H}_{\mathrm{h}} \gamma_{i}^{p, n+1}\right) & =A_{i}, \\
\gamma_{i}^{n+1}-S \Delta t \frac{12}{25} \mathrm{D}_{\mathrm{h}}\left(\frac{1}{\sigma_{i}^{n+1}} \mathrm{D}_{\mathrm{h}} \theta_{i}^{n+1}\right) & =B_{i},
\end{aligned}
$$


where $A_{i}$ and $B_{i}$ depend only on the previous time steps. In particular, when the parametrization is selected with $R \equiv 1$, the previous system can be inverted easily in Fourier space. This is the frame we use in all our computations presented here.

On the other hand the evolution equation for $\sigma$ does not contribute to the high order stiffness. Therefore, an explicit method can be used to update $\sigma$ in time. In our implementation, we employ the following fourth order Adams-Bashforth scheme,

$$
\sigma_{i}^{n+1}=\sigma_{i}^{n}+\frac{\Delta t}{24}\left(55 F_{i}^{n}-59 F_{i}^{n-1}+37 F_{i}^{n-2}-9 F_{i}^{n-3}\right),
$$

where $F_{i}^{n}$ is the right hand side of (25) computed at time $n \Delta t$.

All our computations are for 1-periodic interfaces. The simulations in $[0,1]$ are achieved by a simple scaling of the $2 \pi$-periodic case. All the calculations were performed with double precision on a Sparc10 workstation.

A useful check for the accuracy of the numerics is the monitoring of the energy. For a two-fluid interface, the total perturbation energy given by

$$
\begin{aligned}
E_{T} & =S\left(\int_{0}^{1} \sigma\left(\alpha^{\prime}\right) d \alpha^{\prime}-1\right)+\frac{1}{2} \int_{0}^{1} \gamma(\alpha) \psi(\alpha) d \alpha \\
& +A\left[\int_{0}^{1} \psi(\alpha) \phi_{\alpha}(\alpha) d \alpha+g \int_{0}^{1} y^{2}(\alpha) x_{\alpha}(\alpha) g \alpha\right],
\end{aligned}
$$

where $\phi$ and $\psi$ are the velocity potential and stream function respectively, that is,

$$
\phi(\alpha)+i \psi(\alpha)=\frac{1}{2 \pi i} \int_{0}^{1} \gamma\left(\alpha^{\prime}\right) \log \left[\sin \pi\left(z(\alpha)-z\left(\alpha^{\prime}\right)\right] d \alpha^{\prime},\right.
$$

is conserved in time. $E_{T}$ can be computed with spectral accuracy [4]. In our computations, we monitor the number of digits in the fractional change of energy, that is,

$$
\text { Number of Digits }=-\log _{10} \frac{\left|E_{T}(t)-E_{T}\left(t_{0}\right)\right|}{\left|E_{T}\left(t_{0}\right)\right|},
$$

where $E_{T}(t)$ is the total energy at time $t$.

7.1. An Interfacial Standing Wave. This example illustrates the stability of our method. The situation is nearly that of an air-water interface. We take the Atwood number $A=0.9$, the surface tension coefficient $S=0.01$, the gravity constant $g=1$ and the following initial position and vortex sheet strength for the interface:

$$
\begin{aligned}
& x(\alpha, 0)=\alpha+0.01 \sin (2 \pi \alpha), \\
& y(\alpha, 0)=-0.01 \sin (2 \pi \alpha), \\
& \gamma(\alpha, 0)=0.01 \sin (2 \pi \alpha) .
\end{aligned}
$$

This corresponds to a small perturbation about the equilibrium state. The interface behaves like a standing wave, completing one full oscillation at about $t=2.4$. The position of the interface, as it evolves in time, is plotted in Figure 1. In this computation, $N=128$ and $\Delta t=0.0025$. Figure 2(a) shows the spectrum of $y$ versus the wavenumber $k$, plotted at $t=0,1.2,2.4, \ldots, 12$. The wave performs about 5 complete oscillations by the time $t=12$, that is, after 4800 time steps. The spectra clearly shows that the numerical solution is stable and smooth. In Figure 2(b), we plot the $l^{2}$ norm of the difference in the position variable $y$ between the 

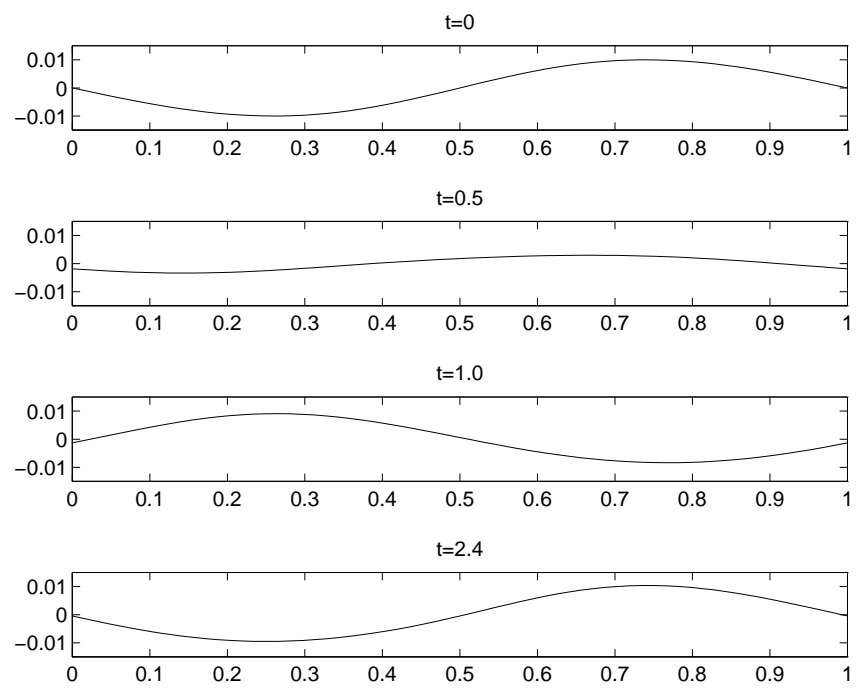

Figure 1. A standing wave for time $t=0,0.5,1.0,2.4 . A=0.9$, $S=0.01$ and $g=1 . N=128$ and $\Delta t=0.0025$.
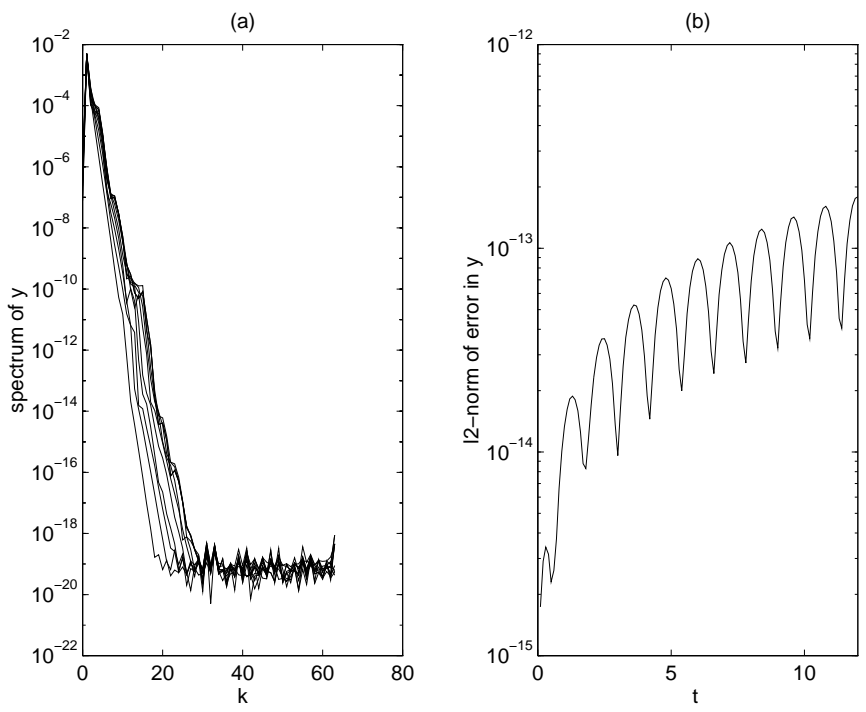

Figure 2. Standing Wave: (a) Spectrum of $y$ at $t=$ $1.2,2.4, \ldots, 12$; (b) $l^{2}$-norm of the difference in $y$ between the numerical solutions for $N=128$ and $N=256$ with $\Delta t=0.0025$.

numerical solutions obtained with $N=128$ and $N=256$. Here $\Delta t=0.0025$. For this simple case in which the amplitude of the wave is small and the interface profile remains smooth, few points are needed to obtain high resolution. Figure 3 shows the number of digits in the fractional change of energy against time, for $N=128$ and $\Delta t=0.0025$. 


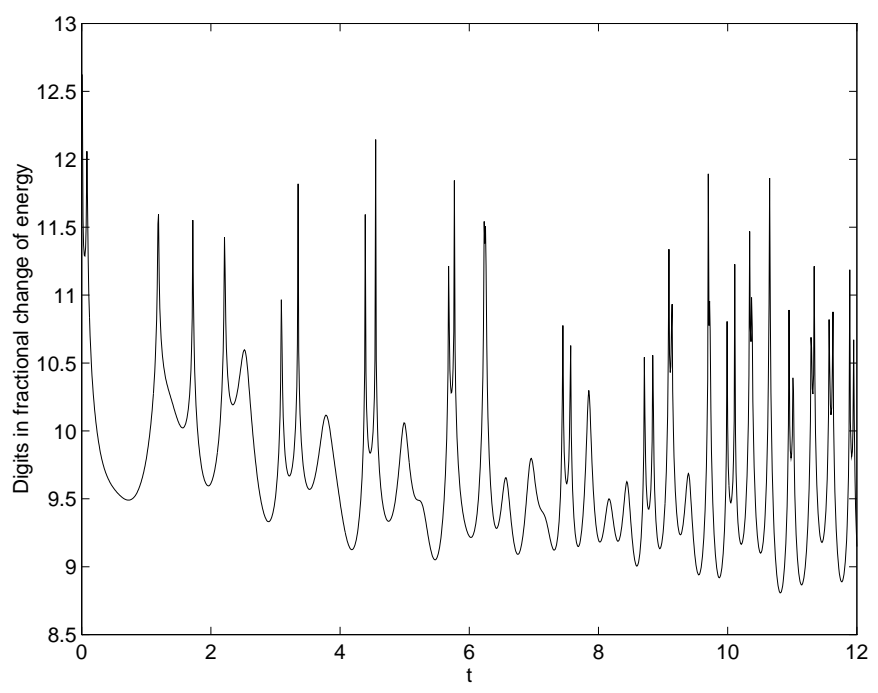

Figure 3. Standing Wave: Numbers of digits in the fractional change of the total energy versus time.

7.2. Unstably Stratified Two-Density Interfacial Flow. One of the classical examples of hydrodynamic instability occurs when a layer of heavy fluid sits on top of a lighter fluid, or equivalently when the interface is accelerated toward the heavy fluid. This is called Raleigh-Taylor Instability [20].

We can simulate this unstably stratified two-density interfacial flow by considering negative values of the product of the Atwood number and the gravity acceleration, that is, $A g<0$, and taking the following initial data

$$
\begin{aligned}
& z(\alpha, 0)=\alpha+i \epsilon \cos (2 \pi \alpha), \\
& \gamma(\alpha, 0)=0 .
\end{aligned}
$$

For our computations we use $\epsilon=0.1$. Figure 4 shows the time evolution of an interface with $A=-0.1, g=10$, and $S=0.005$. For this calculation we use $N=1024$ and $\Delta t=2.5 \times 10^{-4}$. Near $t=0.8$, the interface becomes vertical in two symmetric positions about $x=0.5$. Before $t=0.9$ two small fingers appear and the interface begins to roll up. The fingers in the lighter fluid are thicker than those in the heavy fluid. Capillary waves are produced near $t=1.2$ and move outwards from the centers of roll-up. Figure 5(a) shows the number of digits in the fractional change of energy for $N=1024$ and $N=2048$ plotted against time. There is a sudden loss of accuracy between $t=0.8$ and $t=0.9$ close to the time when the fingers appear. This is probably due to a remnant of Kelvin-Helmholtz instability. From this time on, and up to $t=1.4$, the accuracy stays at about 4.66 digits for $N=1024$ and about 7.2 digits for $N=2048$. Convergence under refinement is illustrated in Figure 5(b) where the $l^{2}$-norm of difference in the $y$ coordinate between both resolutions $N=1024$ and $N=2048$ is plotted against time. Before $t=0.8$, both computations agree within almost 12 digits of accuracy. The spectra of $y$ and $\gamma$ plotted in Figure 6(a)-(b), show clearly the transition that occurs between $t=0.8$ and $t=0.9$ when the sudden loss of accuracy is observed. After $t=0.9$, the spectrum saturates and the Fourier components begin to decrease. 

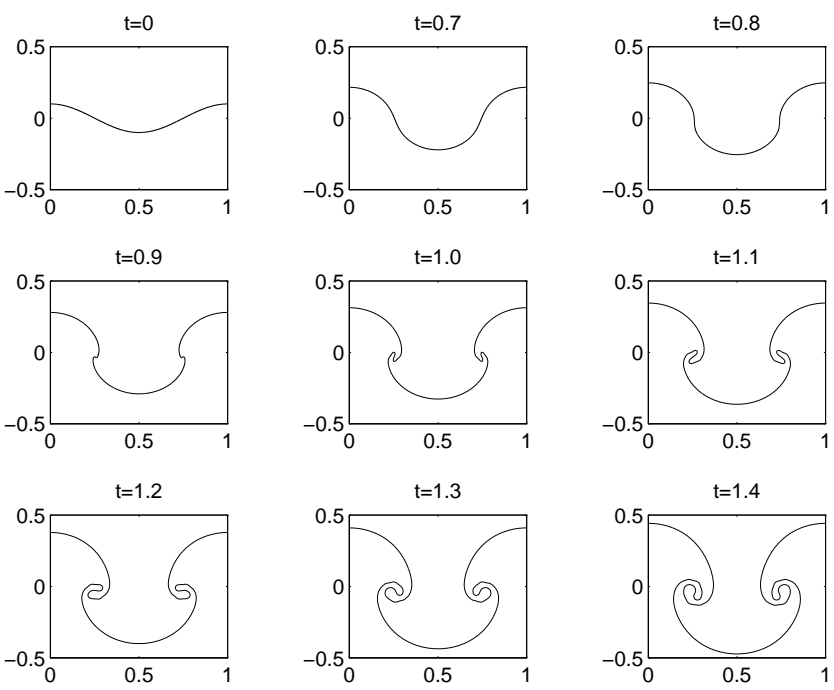

Figure 4. Rayleigh-Taylor Instability: Time evolution of the interface with $A=-0.1$ and $S=0.005$. Computations performed with $N=1024$ and $\Delta t=2.5 \times 10^{-4}$.
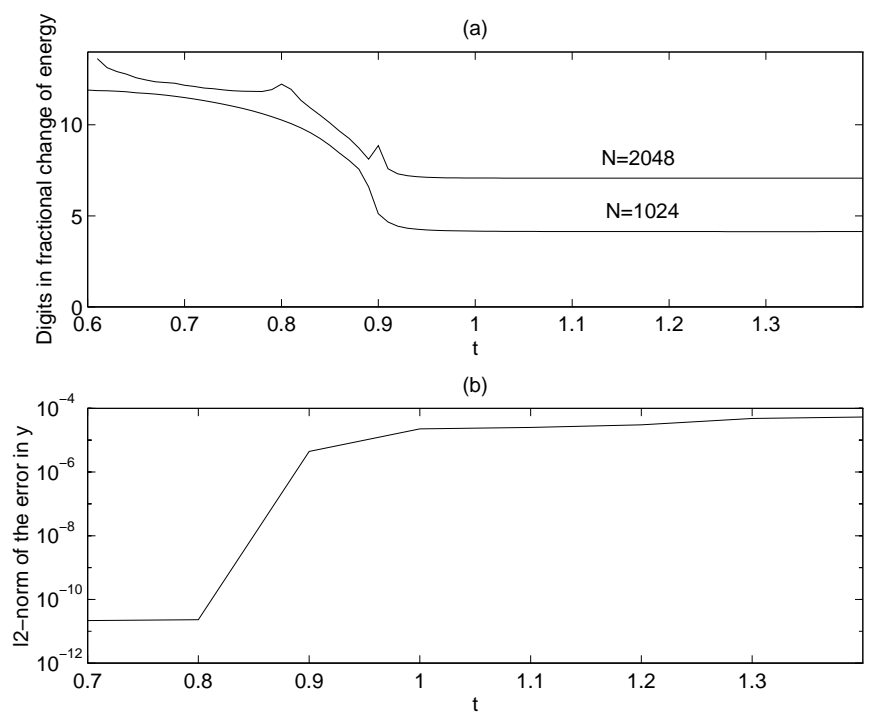

Figure 5. Rayleigh-Taylor Instability: $A=-0.1$ and $S=0.005$. (a) Number of accurate digits in energy for $N=1024$ and $\Delta t=2.5^{-4}$, and $N=2048$ and $\Delta t=1.25^{-4}$. (b) $l^{2}$-norm of the difference in $y$ between the computations obtained with $N=1024$ and $N=2048$. 

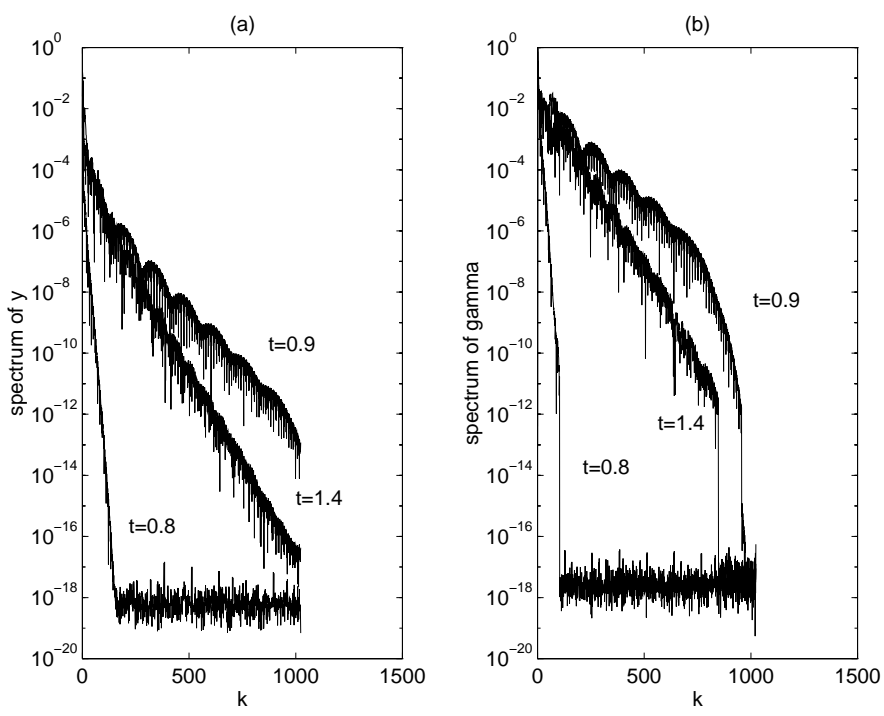

Figure 6. Rayleigh-Taylor Instability: $A=-0.1$ and $S=0.005$. (a) Spectrum of $y$ at $t=0.8,0.9$ and 1.4. (b) Spectrum of $\gamma$ at $t=0.8,0.9$ and 1.4 .
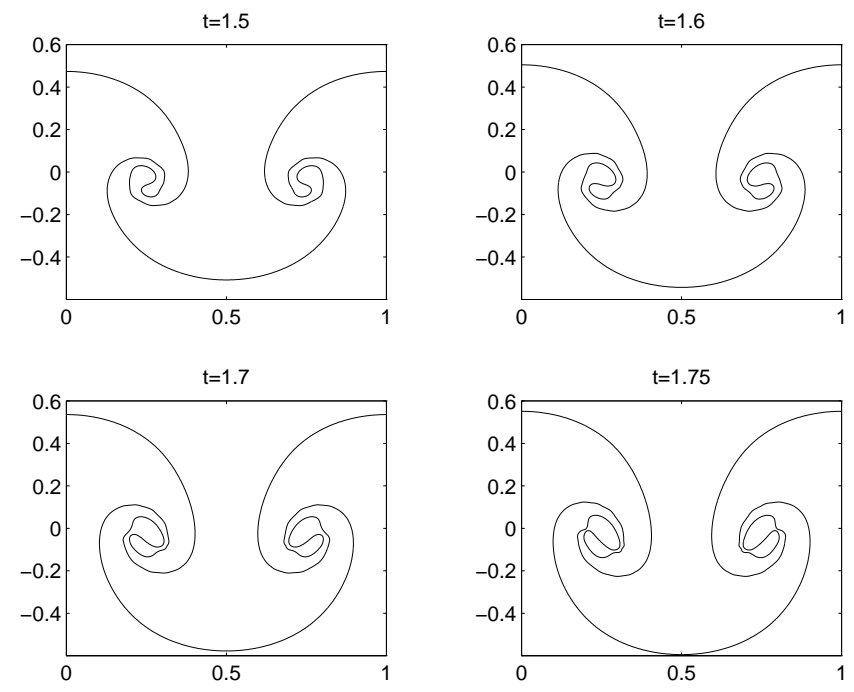

Figure 7. Rayleigh-Taylor Instability: $A=-0.1$ and $S=0.005$.

Sequence of interface positions. $N=2048$ and $\Delta t=1.25 \times 10^{-4}$.

The computations can proceed further with still good accuracy for $N=2048$. Figure 7 shows a sequence of the interface position in time from $t=1.5$ to $t=1.75$. Note that the tips of the fingers broaden as they continue to roll, and that the interface bends towards the tip of the fingers. At $t=1.75$, the minimum separation between the tip of the fingers and the opposite side of the interface is about 0.016 
and the interface length is about 1.68. The minimum distance continues to approach zero. By $t=1.785$ (Figure 8 ), the separation is approximately $5 \times 10^{-4}$ and the computations have to be stopped soon after this time.

This process of bubble formation through self-intersection of a fluid interface has been observed by HLS [23] for a vortex sheet. Based on their numerical results and a local model, HLS have suggested that the interface minimum separation decreases proportional to $\left(t_{c}-t\right)^{2 / 3}$, reaching zero at finite $t=t_{c}$. In Figure 9 , we compare the computed minimum separations $d_{i}$, represented as circles in the plot, and a fitted curve of the form $d(t)=C\left(t_{c}-t\right)^{2 / 3}$ as a solid line. There is good agreement between the values $d_{i}$ and $d\left(t_{i}\right)$ up to $t=1.76$. Beyond this time, we see a deviation of the computed values $d_{i}$ from the fitted curve. However, the number of digits in the fractional change of energy continues to decrease from about 4.7 at $t=1.75$ (Figure 10) to less than 3 digits at the final computed time $t=1.785$. We believe the accuracy is not high enough to resolve a separation of order $O\left(5 \times 10^{-3}\right)$ or smaller (Figure 10) and thus, more resolution is needed near the pinching time to give a more conclusive answer about the rate at which the minimum interface separation goes to zero. High accuracy can be achieved by selecting appropriately the parametrization function $R$. This has already been done by HLS [23] for accurate vortex sheet computations.

Roll-up has been also observed in other numerical simulations of Rayleigh-Taylor instability such as the early marker-in-cell computations of Daly [18], the vortexin-cell method of Tryggvason [44] and the projection method for variable density of Bell and Marcus [12]. However, unlike these methods, the only regularization we use here is surface tension. Thus, the structure of the roll-up and the formation of trapped bubbles through self-intersection of the interface are genuinely due to surface tension. Another approach for numerical simulation of free boundary problems is the level set method introduced by Osher and Sethian [33]. Recent computations using this technique include the motion of bubbles in water and the rising of fluid drops in air $[16,42]$.

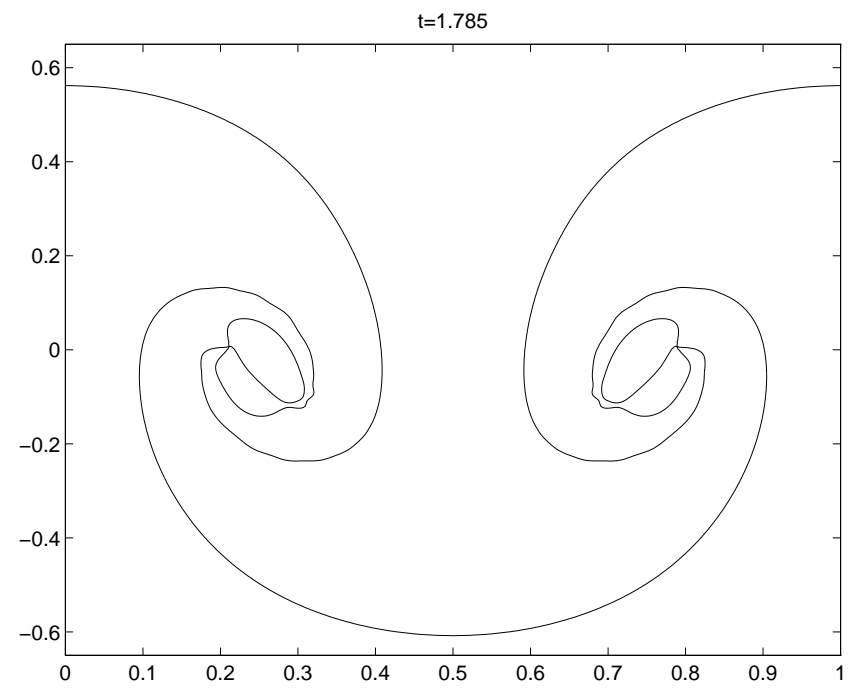

Figure 8. Rayleigh-Taylor Instability: $A=-0.1$ and $S=0.005$. Interface position at $t=1.785$ 


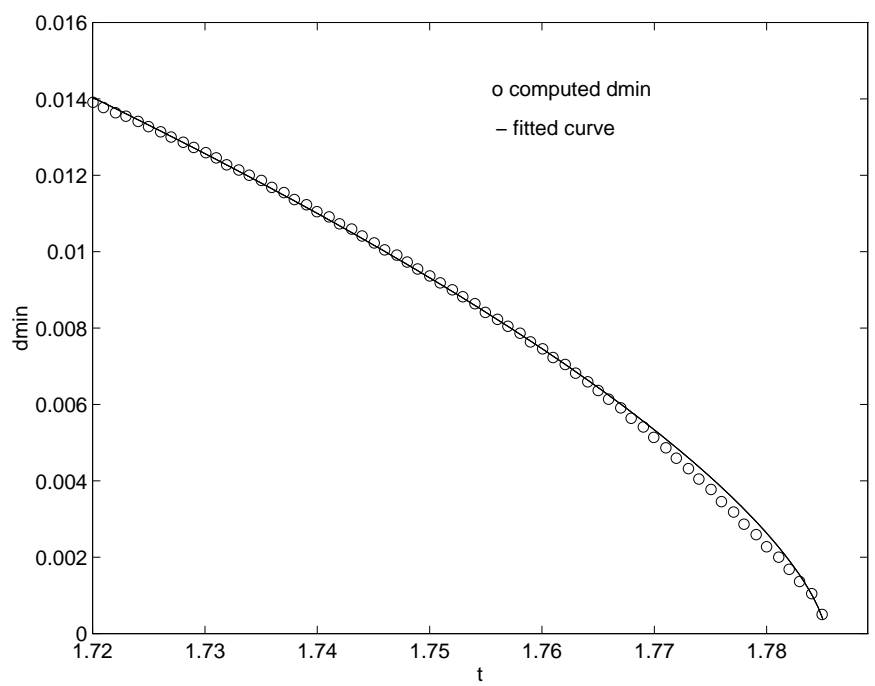

Figure 9. Rayleigh-Taylor Instability: $A=-0.1$ and $S=0.005$. Minimum separation against time. The circles are the minimum distance between the tip of the fingers and the opposite side of the interface computed with $N=2048$ and $\Delta t=1.25 \times 10^{-4}$. The solid line is a fitted curve of the form $d(t)=C\left(t_{c}-t\right)^{2 / 3}$.

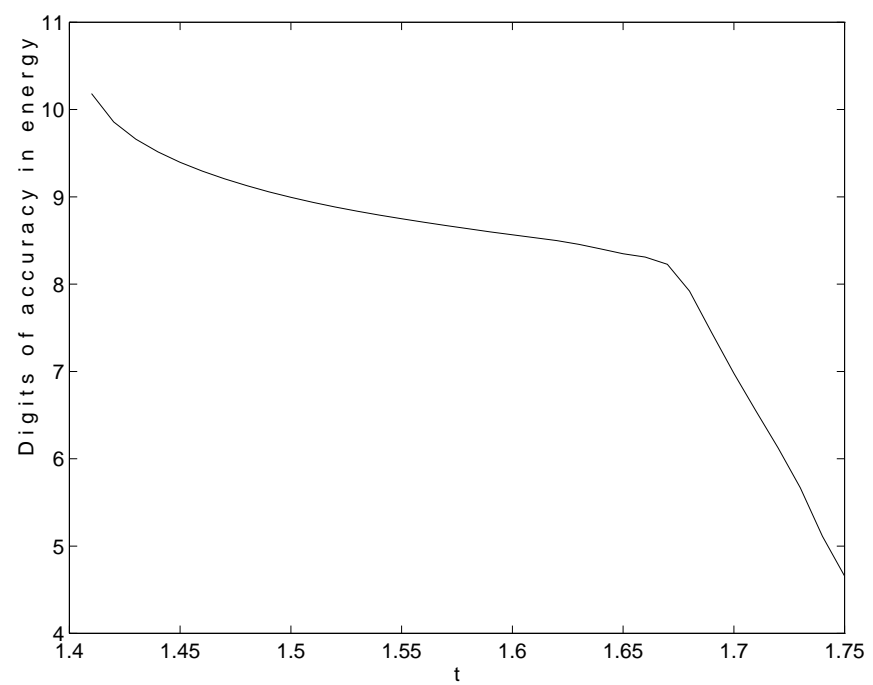

Figure 10. Rayleigh-Taylor Instability: $A=-0.1$ and $S=0.005$. Number of accurate digits in the fractional change of energy against time. 
7.3. Drop Formation in a Single Fluid. A drop falling from a faucet is a common example in which there is a change of topology in the interface and physical quantities become discontinuous.

Pullin [35] has provided indications that surface tension may cause drop formation in a falling fluid. However, his computations could not go very far due to numerical instability.

To simulate the process of drop formation in a falling single fluid we take $A=1$ and negative gravity constant in our method for two-density fluid interfaces. We remark here that, from the computational point of view, it is more efficient to use a velocity potential water waves formulation for which we have also designed and analyzed a stable method using the arclength stretching-tangent angle setting [15]. Numerically there is no problem in taking $A=1$ or $A=-1$ in our two-density method, and the numerical values from both schemes are the same within machine precision.

In the numerical example we present next, $g=-10$ and the surface tension coefficient is $S=0.5$. We use the same initial data as in our Rayleigh-Taylor examples, but with a change of sign in the $y$ coordinate.

A sequence of the interface position for different times is presented in Figures 1112. As the fluid falls, the interface stretches forming a pendant drop. By the time $t=1.3$ (Figure 12) the fluid has a long neck whose minimum thickness decreases in time. At time $t=1.5$, the interface length is more than 10 times its initial length and the minimum thickness of the fluid neck, just above the drop is about 0.02 . The interface is about to pinch off.

Figure 13 shows an apparent linear decrease of the neck thickness, and thus, possibly a different rate than that in the Rayleigh-Taylor roll-up. Of course, we still do not know the behavior of the thickness closer to the pinching.
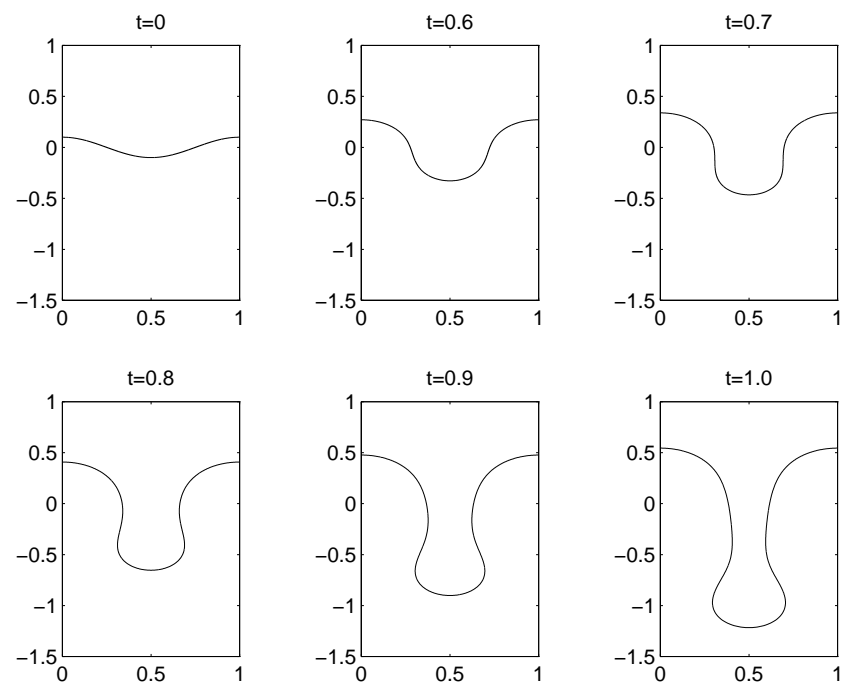

Figure 11. Falling of a Single Fluid. Sequence of interface positions at different times $t . \quad \tau=0.25, g=-10, N=1024$ and $\Delta t=2.5 \times 10^{-4}$. 

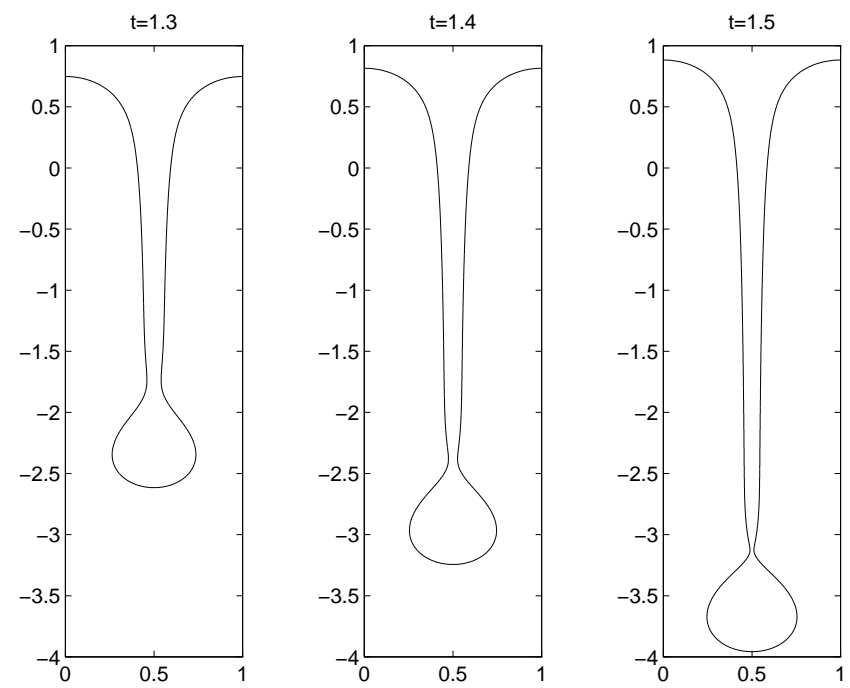

Figure 12. Falling of a Single Fluid: Sequence of interface positions at times $t=1.3, t=1.4$, and $t=1.5 . g=-10, \tau=0.25$, $N=2048$ and $\Delta t=1.25 \times 10^{-4}$.

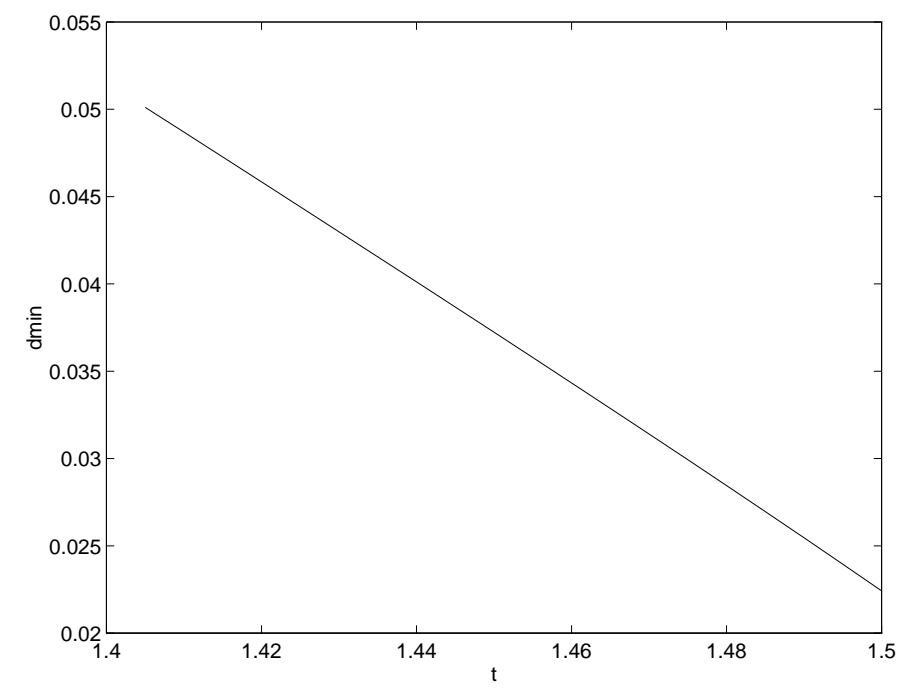

Figure 13. Falling of a Single Fluid: Minimum thickness in the neck of the fluid. $N=2048$ and $\Delta t=1.25 \times 10^{-4}$.

The accuracy in the energy is plotted in Figure 14, from $t=1.4$ to $t=1.5$. The calculation was performed with $N=2048$ and $\Delta t=1.25 \times 10^{-4}$. Before $t=1.4$ the interface is well resolved even with $N=1024$ and the accuracy in the energy is about 9 digits. Close to $t=1.46$ the accuracy decreases as the thickness of 


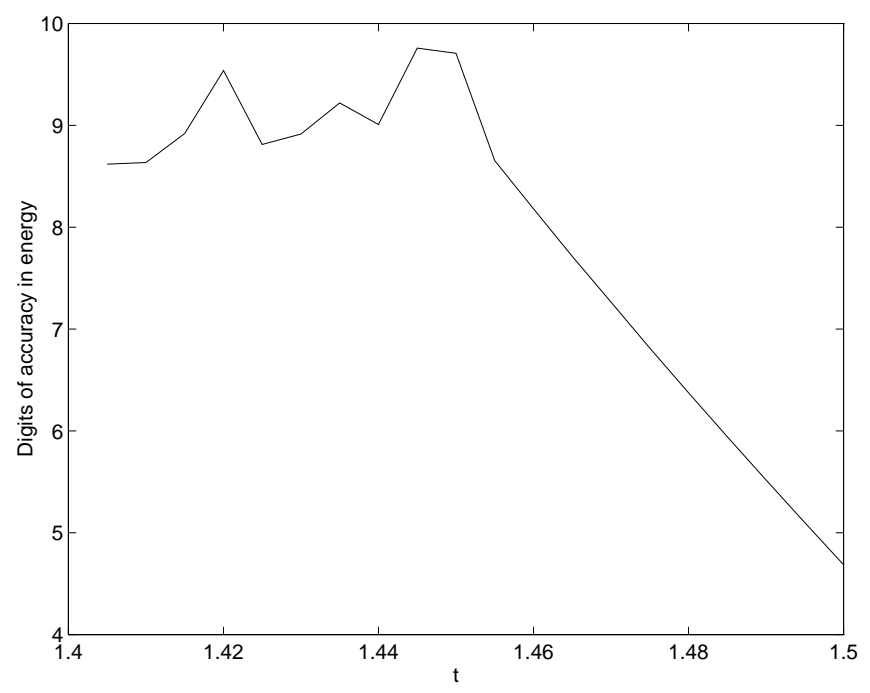

Figure 14. Falling of a Single Fluid: Number of accurate digits in the fractional change of energy against time. $N=2048$ and $\Delta t=1.25 \times 10^{-4}$.

the fluid neck approaches zero and the interface continues to stretch, leaving fewer Lagrangian grid points near the regions of high curvature. An adaptive refinement through the selection of a time-dependent parametrization function $R$ may solve this difficulty. We will implement this idea in the future [14].

\section{ACKNOWLEDGEMENTS}

We would like to acknowledge Professors Thomas Beale, John Lowengrub and Michael Shelley for a number of interesting discussions during the preparation of this work.

\section{REFERENCES}

1. U. M. Ascher, S. J. Ruuth, and B. Wetton, Implicit-explicit methods for time-dependent partial differential equations, SIAM J. Numer. Anal. 32 (1995), no. 3, 797-823. MR 96j:65076

2. G. Baker, Generalized vortex methods for free-surface flows, Waves on Fluid Interfaces (R. Meyer, ed.), Univ. Wisc. Press, 1983, pp. 53-81.

3. G. R. Baker and M. J. Shelley, On the connection between thin vortex layers and vortex sheets, J. Fluid Mech. 215 (1990), 161-194. MR 91i:76010

4. Gregory Baker and André Nachbin, Stable methods for vortex sheet motion in presence of surface tension, Submitted to J. Comp. Phys.

5. Gregory R. Baker, Daniel I. Meiron, and Steven A. Orszag, Vortex simulations of the RayleighTaylor instability, Phys. Fluids 23 (1980), 1485-1490.

6. Generalized vortex methods for free-surface flow problems, J. Fluid Mech. 123 (1982), 477-501. MR 84a:76002

7. J. T. Beale, Thomas Y. Hou, and John S. Lowengrub, Convergence of a boundary integral method for water waves, SIAM J. Num. Anal. 33 (1996), 1797-1843. CMP 97:02

8. ㄴ The stability of two-fluid flows with surface tension, part 1: Growth rates for the linear motion far from equilibrium; part 2: Convergence of suitably modified vortex methods, In preparation. 
9. _ Growth rates for the linearized motion of fluid interfaces away from equilibrium, Comm. Pure Appl. Math. 46 (1993), 1269-1301. MR 95c:76016

10. - On the well-posedness of two fluid interfacial flows with surface tension, Singularities in Fluids, Plasmas and Optics (London) (R. C. Caflisch and G. C. Papanicolaou, eds.), Kluwer Academic, 1993.

11. J. T. Beale, Thomas Y. Hou, John S. Lowengrub, and Michael J. Shelley, Spatial and temporal stability issues for interfacial flows with surface tension, Math. Comput. Modelling 20 (1994), no. 10/11, 1-27. MR 95i:76077

12. J. B. Bell and D. L. Marcus, A second-order projection method for variable-density flows, J. Comp. Phys. 101 (1992), 334-348.

13. J. U. Brackbill, D. B. Kothe, and C. Zemach, A continuum method for modeling surface tension, J. Comp. Phys. 100 (1992), 335-354. MR 93c:76008

14. H. D. Ceniceros and T. Y. Hou, Numerical studies of surface tension effects in interfacial flows, In preparation.

15. Héctor D. Ceniceros, Convergence of a reformulated boundary integral method for two fluid interfaces with surface tension, Ph.D. thesis, New York University, May 1995.

16. Y. C. Chang, T. Y. Hou, B. Merriman, and S. Osher, Eulerian capturing methods based on a level set formulation for incompressible fluid interfaces, J. Comp. Phys. 124 (1996), 449-464. MR 97a:76087

17. W. S. Dai and M. J. Shelley, A numerical study of the effect of surface tension and noise on an expanding Hele-Shaw bubble, Phys. Fluids A 5 (1993), no. 9, 2131-2146.

18. Bart J. Daly, Numerical study of the effect of surface tension on interface instability, Phys. Fluids 12 (1969), no. 7, 1340-1354.

19. J. W. Dold, An efficient surface-integral algorithm applied to unsteady gravity waves, J. Comp. Phys. 103 (1992), 90-115. MR 93g:76091

20. P. G. Drazin and W. H. Reid, Hydrodynamic stability, Cambridge monographs on mechanics and applied mathematics, Cambridge University Press, New York, 1981. MR 82h:76021

21. R. Goldstein and D. M. Petrich, The Korteweg-de Vries heirarchy as dynamics of closed curves in the plane, Phys. Rev. Let. 67 (1991), 3203-3206. MR 92g:58050

22. Gunther Hammerlin and Karl Heinz Hoffmann, Numerical mathematics, Springer-Verlag, New York, 1991, Translation of: Numerische Mathematik. MR 92d:65001

23. Thomas Y. Hou, John S. Lowengrub, and Michael J. Shelley, The roll-up and self-intersection of vortex sheets under surface tension, Preprint. Courant Institute.

24. _ Removing the stiffness from interfacial flows with surface tension, J. Comp. Phys. 114 (1994), 312-338. MR 95e:76069

25. D.A. Kessler, J. Koplik, and H. Levine, Geometrical models of interface evolution. II, Phys. Rev. A 30 (1984), 3161-3174.

26. Robert Krasny, Desingularization of periodic vortex sheet roll-up, J. Comp. Phys. 65 (1986), 292-313.

27. - A study of singularity formation in a vortex sheet by the point vortex approximation, J. Fluid Mech. 167 (1986), 65-93. MR 87g:76028

28. L. D. Landau and E. M. Lifshitz, Fluid mechanics, Pergamon Press, London, 1959, Translated from the Russian. MR 21:6839

29. J. S. Langer, Instabilities and pattern formation in crystal growth, Rev. Modern Phys. 52 (1980), 1-28

30. M. S. Longuet-Higgins and E. D. Cokelet, The deformation of steep surface waves on water I. a numerical method of computation, Proc. R. Soc. Lond. A. 350 (1976), 1-26. MR 53:15091

31. A. I. Markushevich, Theory of functions of a complex variable, second ed., Chelsea Publishing Company, New York, 1977. MR 56:3258

32. E. Meiburg and G. M. Homsy, Nonlinear unstable viscous fingers in Hele-Shaw flows .2. Numerical simulation, Phys. Fluids 31 (1988), no. 3, 429-439.

33. S. Osher and J. Sethian, Fronts propagating with curvature-dependent speed:Algorithms based on Hamilton-Jacobi formulations, J. Comp. Phys. 79 (1988), 12-49. MR 89h:80012

34. C. Pozrikidis, Boundary integral and singularity methods for linearized viscous flow, Cambridge University Press, 1992. MR 93a:76027

35. D. I. Pullin, Numerical studies of surface-tension effects in nonlinear Kelvin-Helmholtz and Rayleigh-Taylor instability, J. Fluid Mech. 119 (1982), 507-532. 
36. R.H. Rangel and W.A. Sirignano, Nonlinear growth of Kelvin-Helmholtz instability: Effect of surface tension and density ratio, Phys. Fluids 31 (1988), no. 7, 1845-1855.

37. A. J. Roberts, A stable and accurate numerical method to calculate the motion of a sharp interface between fluids, IMA J. Appl. Math. 31 (1983), 13-35.

38. M. J. Shelley, A study of singularity formation in vortex sheet motion by a spectrally accurate vortex method, J. Fluid Mech. 244 (1992), 493-526. MR 93g:76035

39. A. Sidi and M. Israeli, Quadrature methods for periodic singular and weakly singular Fredholm integral equations, J. Sci. Comp. 3 (1988), 201-231. MR 90e:65194

40. J. Strain, A boundary integral approach to unstable solidification, J. Comp. Phys. 85 (1989), 342-389. MR 90k:80015

41. Gilbert Strang, Accurate Partial Difference Methods. II. Non-Linear Problems, Numerische Mathematik 6 (1964), 37-46. MR 29:4215

42. M. Sussman, P. Smereka, and S. Osher, A level set approach for computing solutions to incompressible 2-phase flow, J. Comp. Phys. 114(1) (1994), 146-159.

43. Eitan Tadmor, Stability analysis of finite-difference, pseudospectral and Fourier-Galerkin approximations for time-dependent problems, SIAM Review 29 (1987), no. 4, 525-555. MR 88m:65136

44. Grétar Tryggvason, Numerical simulations of the Rayleigh-Taylor instability, J. Comp. Phys. 75 (1988), 253-282.

Department of Applied Mathematics, California Institute of Technology, Pasadena, CALIFORNIA 91125

Current address: Centro de Investigación en Computación, Instituto Politécnico Nacional, Col. Lindavista, Mexico City, Mexico 07300.

E-mail address: hdc@jsbach.cic.ipn.mx

Department of Applied Mathematics, California Institute of Technology, Pasadena, CALIFornia 91125

E-mail address: hou@ama.caltech.edu 